\title{
Existência de soluções para equações integro-diferenciais neutras
}

\author{
José Paulo Carvalho dos Santos
}

Orientador: Eduardo Alex Hernández Morales

Tese apresentada ao Instituto de Ciências Matemáticas e de Computação - ICMC-USP, como parte dos requisitos para obtenção do título de Doutor em Ciências - área Matemática.

USP - São Carlos

Março/2006 

"Retroceder nunca...

render-se jamais!"

Filme: "No Retreat, No Surrender". 



\section{Agradecimentos}

Agradeço a Deus pela saúde e perseverança.

Ao Prof. Eduardo Hernandez, por ter aceito me orientar no doutorado e pela forma segura e dedicada que conduziu todo processo de Tese.

À Ni, por tornar minha vida tão mais feliz.

Ao meu irmão Luis Antônio, pelos conselhos valiosos e pela grande ajuda financeira durante os primeiros anos do doutorado, período em que estava sem bolsa.

Aos Meus pais Carmo e Aparecida e minhas irmãs Ana Maria e Cláudia, pelo amor, apoio e orações.

Ao Professor Ladeira por ter me apresentado ao Professor Eduardo Hernandez, o que tornou meu doutorado possível.

Aos funcionários e funcionárias do ICMC-USP, pela dedicação que empregam no desenvolvimento de seu trabalho.

Aos amigos, pelo apoio e pelos muitos momentos divertidos, os quais não irei esquecer.

À Capes, pelo suporte financeiro. 



\section{Abstract}

In this work we study the existence of mild, semi-classical and classical solution, concepts introduced be later for a class of abstract neutral functional integrodifferential systems with unbounded delay in the form

$$
\begin{aligned}
\frac{d}{d t} \mathcal{D}\left(t, x_{t}\right)= & A \mathcal{D}\left(t, x_{t}\right)+\int_{0}^{t} B(t-s) \mathcal{D}\left(s, x_{s}\right) d s+g\left(t, x_{t}\right), \quad t \in(0, a), \\
x_{0}=\varphi & \in \mathcal{B}, \\
\frac{d}{d t}\left(x(t)+F\left(t, x_{t}\right)\right) & =A x(t)+\int_{0}^{t} B(t-s) x(s) d s+G\left(t, x_{t}\right), \quad t \in(0, a), \\
x_{0} & =\varphi \in \mathcal{B},
\end{aligned}
$$

where $A: D(A) \subset X \rightarrow X$ is a closed linear densely defined operator in a Banach space $X$, each $B(t): D(B(t)) \subset X \rightarrow X$ is a closed linear operator, the history $x_{t}:(-\infty, 0] \rightarrow X$, $x_{t}(\theta)=x(t+\theta)$, belongs to some abstract phase space $\mathcal{B}$ defined axiomatically and $\mathcal{D}, F, g$ : $[0, a] \times \mathcal{B} \rightarrow X$ are appropriate functions.

To establish some of our results, we studied the existence and qualitative properties of a resolvent of bounded linear operators $(R(t))_{t \geq 0}$, for a system in the form

$$
\begin{aligned}
\frac{d}{d t}\left(x(t)+\int_{0}^{t} N(t-s) x(s) d s\right) & =A x(t)+\int_{0}^{t} B(t-s) x(s) d s, \quad t \in(0, a), \\
x(0) & =x_{0},
\end{aligned}
$$

where $(N(t))_{t \geq 0}$ is a family of bounded linear operators on $X$. We mention that this class of system arise in the study of heat conduction in material with fading memory. 



\section{Resumo}

Neste trabalho estudaremos a existência de soluções fracas, semi-clássicas e clássicas, conceitos introduzidos no texto para uma classe de sistemas integro-diferenciais do tipo neutro com retardamento não limitado modelados na forma

$$
\begin{aligned}
\frac{d}{d t} \mathcal{D}\left(t, x_{t}\right)= & A \mathcal{D}\left(t, x_{t}\right)+\int_{0}^{t} B(t-s) \mathcal{D}\left(s, x_{s}\right) d s+g\left(t, x_{t}\right), \quad t \in(0, a), \\
x_{0}=\varphi & \in \mathcal{B}, \\
\frac{d}{d t}\left(x(t)+F\left(t, x_{t}\right)\right) & =A x(t)+\int_{0}^{t} B(t-s) x(s) d s+G\left(t, x_{t}\right), \quad t \in(0, a), \\
x_{0} & =\varphi \in \mathcal{B},
\end{aligned}
$$

onde $A$ é um operador linear fechado densamente definido em um espaço de Banach $X$, cada $B(t): D(B(t)) \subset X \rightarrow X, t \geq 0$ é um operador linear fechado, a história $x_{t}:(-\infty, 0] \rightarrow$ $X, x_{t}(\theta)=x(t+\theta)$, pertence a um espaço de fase abstrato $\mathcal{B}$ definido axiomaticamente $\mathrm{e}$ $\mathcal{D}, F, g, G:[0, a] \times \mathcal{B} \rightarrow X$ são funções apropriadas.

Para obter alguns de nossos resultados, estudamos a existência e propriedades qualitativas de uma família resolvente de operadores lineares limitados $(R(t))_{t \geq 0}$, para o sistema integro-diferencial

$$
\begin{aligned}
\frac{d}{d t}\left(x(t)+\int_{0}^{t} N(t-s) x(s) d s\right) & =A x(t)+\int_{0}^{t} B(t-s) x(s) d s, \quad t \in(0, a), \\
x(0) & =x_{0},
\end{aligned}
$$

onde $(N(t))_{t \geq 0}$ é uma família de operadores lineares limitados em $X$. Mencionamos que este tipo de sistemas aparece no estudo da condução de calor em materiais com memória amortecida. 



\section{Sumário}

Introdução xiii

1 Preliminares 1

$1.1 \quad$ Semigrupos de operadores lineares $\ldots \ldots \ldots \ldots$. . . . . . . . . . . 1

$1.2 \quad$ Operador resolvente para equações integro-diferenciais $\ldots \ldots \ldots$. . . . . . 6

1.3 Espaços de fase abstratos e alguns Teoremas . . . . . . . . . . . . . . . . . 10

\begin{tabular}{|lll}
2 & Existência de soluções para uma equação integro-diferencial & 13
\end{tabular}

2.1 Existência de soluções fracas $\ldots \ldots \ldots \ldots \ldots$

2.2 Resultados de regularidade . . . . . . . . . . . . . . . . . . . . 22

2.2 .1 Existência de solução semi-clássica . . . . . . . . . . . . . . . . . . . 22

2.2 .2 Existência de solução clássica . . . . . . . . . . . . . . . . . 25

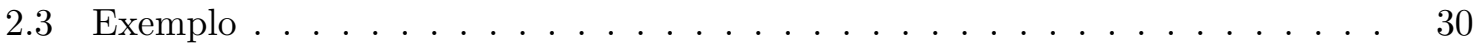

3 Existência de soluções para um sistema integro-diferencial 33

3.1 Existência de soluções fracas $\ldots \ldots$. . . . . . . . . . . . . . . . . 33

3.2 Resultados de regularidade $\ldots \ldots \ldots \ldots \ldots$. . . . . . . . . . . . . 43

3.2.1 Existência de soluções semi-clássicas . . . . . . . . . . . . . . . . . . 44

$3.2 .2 \quad$ Existência de solução clássica $\ldots \ldots \ldots$. . . . . . . . . . . . . 48

3.3 Exemplo . . . . . . . . . . . . . . . . . . . . . . . 56

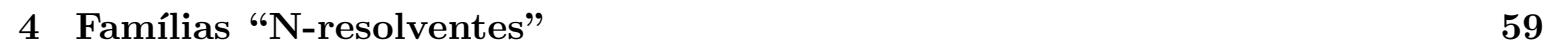

4.1 Preliminares . . . . . . . . . . . . . . . . . . . . . . . . . . . 60

4.2 Existência de uma família N-resolvente . . . . . . . . . . . . . . . . . . . . . 61

$4.3 \quad \mathrm{O}$ problema integro-diferencial não homogêneo $\ldots \ldots \ldots \ldots$. . . . . . . 81

4.4 Aplicações a equações neutras com retardamento não limi- tado . . . . . . . . . 90 
\begin{tabular}{ll}
\hline Referências Bibliográficas & 95
\end{tabular} 


\section{Introdução}

Nas últimas décadas o estudo de sistemas com memória tem recebido muita atenção devido, fundamentalmente, ao fato de que o retardo temporal tem uma influência significativa sobre o comportamento qualitativo do sistema. A maior parte da literatura relativa a este assunto está relacionada com o problema de Cauchy abstrato

$$
\begin{aligned}
\dot{u}(t) & =F\left(t, u_{t}\right), \\
u_{\sigma} & =\varphi \in C\left([-r, 0]: \mathbb{R}^{n}\right) .
\end{aligned}
$$

Como referências para este tipo de equações citamos Hale \& Lunel [29] e Hale \& Kato [30]. Sistemas com retardamento não limitado e valores em $\mathbb{R}^{n}$ também são considerados, veja entre outros, Corduneanu \& Lakshmikanthan [16] e o livro Hino, Murakami \& Naito [49].

Como a solução de (1)-(2) assume valores em um espaço de dimensão finita, o sistema (1)-(2) não permite o estudo de equações diferenciais parciais, deixando de lado muitos sistemas diferenciais interessantes e importantes. Este fato motivou muitos pesquisadores a estudar uma classe de sistemas abstratos descritos na forma

$$
\begin{aligned}
\frac{d}{d t}\left(u(t)+f\left(t, u_{t}\right)\right) & =A u(t)+g\left(t, u_{t}\right), \quad t \in[\sigma, \sigma+a] \\
u_{0} & =\varphi \in \mathcal{B},
\end{aligned}
$$

onde $A$ é o gerador infinitesimal de um semigrupo fortemente contínuo de operadores lineares $(T(t))_{t \geq 0}$ definido sobre um espaço de Banach $X$ e $\mathcal{B}$ é um espaço de fase definido de maneira axiomática. No que segue, nos referimos a este sistema como " Abstract Neutral Functional Differential Equations ".

O sistema diferencial (3)-(4) é uma generalização do sistema clássico onde $f \equiv 0$, o qual tem sido extensamente estudado, veja entre outros Travis \& Weeb [67, 68, 69] e Wu [72]. Mas o modelo (3)-(4) é mais que uma simples generalização abstrata. " Abstract Neutral Functional Differential Equations" aparecem no estudo de diferentes problemas aplicados. O 
sistema (3), por exemplo, aparece na teoria desenvolvida por Gurtin \& Pipkin [27] e Nunziato 62] para a descrição da condução de calor em materiais com memória amortecida. Na teoria de condução de calor é assumido, de maneira geral, que a energia e o fluxo de calor dependem linearmente da temperatura $u(\cdot)$ e do gradiente $\nabla u(\cdot)$. Nestas condições, a clássica equação do calor descreve de maneira satisfatória a evolução da temperatura em diferentes tipos de materiais. Esta situação é diferente em materiais com memória amortecida ( materials with fading memory ). Neste tipo de material, veja [27, 62], a energia interna do material e o fluxo de tempertura são funcionais da $u(\cdot)$ e do gradiente de $u(\cdot)$, respectivamente. Um modelo amplamente aceito para descrever o fluxo de calor em materiais com memória amortecida, veja [12, 13, 56, 62, 65], é o sistema diferencial

$$
\begin{aligned}
\frac{d}{d t}\left[c_{0} u(t, x)+\int_{-\infty}^{t} K_{1}(t-s) u(s, x) d s\right] & =c_{1} \Delta u(t, x)+\int_{-\infty}^{t} K_{2}(t-s) \Delta u(s, x) d s, \\
u(t, x) & =0, \quad t \geq 0, \quad x \in \partial \Omega .
\end{aligned}
$$

Neste sistema, $\Omega \subset \mathbb{R}^{n}$ é um aberto limitado e com fronteira regular; $(t, x) \in[0, \infty) \times \Omega ; u(\cdot)$ representa a temperatura em $x$ no tempo $t ; c_{1}, c_{2}$ são constantes positivas com significado físico e $K_{i}: \mathbb{R} \rightarrow \mathbb{R}, i=1,2$, são funções apropriadas.

Outra motivação importante para o desenvolvimento da teoria de equações funcionais do tipo neutro é o estudo de uma classe de equações parciais do tipo hiperbólico com certas condições de fronteira não lineares, que surgem no estudo de linhas de transmissão. Com relação a isto, veja os trabalhos de Abolinia \& Mishkis [1], Brayton [10], Brayton \& Moser [11, Cooke \& Krumme [15], Cruz \& Hale [17, Lopes [52, 53], Hale [54] e Wu \& Xia [73, 74] para detalhes relativos à história, referências e o estado atual do tema.

Em Wu and Xia [73], a partir de um sistema ordinário do tipo neutro, os autores deduzem a seguinte equação escalar do tipo neutro em derivadas parciais definida sobre o círculo unitário

$$
\begin{aligned}
\frac{\partial}{\partial t} D\left(u_{t}\right) & =k \frac{\partial}{\partial \xi} D\left(u_{t}\right)+g\left(t, u_{t}\right) \\
u_{\sigma} & =\varphi \in C\left([-r, 0]: C\left(S^{1}: \mathbb{R}\right)\right) .
\end{aligned}
$$

Neste sistema, $k$ é uma constante positiva e $(D \phi)(s)=\phi(0)(s)-\int_{-r}^{0}[d \eta(\theta)] \phi(\theta)(s)$ onde $\eta$ é uma função de variação limitada não atômica em zero. Esta equação neutra foi investigada por Hale em [54]. Neste trabalho, Hale estabelece uma pequena teoria para o sistema, considerando a existência e unicidade de soluções, propriedades condensantes do operador solução, bifurcação de Hopf e estabilidade de órbitas periódicas. 
Observe que o sistema neutro (6) pode ser descrito por meio do problema de Cauchy abstrato

$$
\begin{aligned}
\frac{d}{d t}\left(D\left(t, u_{t}\right)\right) & =A D\left(t, u_{t}\right)+g\left(t, u_{t}\right), \\
u_{0} & =\varphi \in \mathcal{B},
\end{aligned}
$$

onde $A$ é o gerador infinitesimal de um $C_{0}$-semigrupo e $\mathcal{B}$ é um espaço de fase apropriado. Com relação a este tipo de sistema mencionamos Dakto [19] e Addimy [2, 3, 4, 5].

Em relação a sistemas abstratos do tipo neutro de primeira ordem modelados como (3)-(4) ou (7)-(8) citamos entre outros trabalhos, [2, 3, 4, 5, 22, 33, 45, 46] para existência de soluções fracas; [5, 45] para regularidade de soluções fracas; [2] para estabilidade de soluções; [32, 35, 34] para existência de soluções periódicas; [36] para a existência de soluções quase e assintoticamente quase periódicas; [37, 38, 39] para soluções pseudo quase periódicas; [40, 41] para sistemas impulsivos e [22, 43] para sistemas com condições não locais. É interessante citar também [46, 42], onde são feitas algumas correções de recentes trabalhos tratando sobre a existência e controlabilidade exata de soluções associadas a sistemas do tipo neutro.

Embora haja extensa literatura para sistemas neutros, o estudo da existência e propriedades qualitativas de soluções de sistemas neutros do tipo integro-diferencial são tópicos até agora não desenvolvidos. Este fato e as interessantes aplicações relacionadas ao problema de condução de calor em materias com memória são as principais motivações de nosso trabalho.

Esta Tese possui quatro capítulos. No Capítulo1 são introduzidas de maneira resumida as diferentes ferramentas técnicas que nos auxiliaram na obteção de nossos resultados.

No Capítulo 2 estudamos diferentes questões relacionadas com a existência e regularidade de soluções para o sistema integro-diferencial

$$
\left\{\begin{aligned}
\frac{d}{d t} \mathcal{D}\left(t, u_{t}\right) & =A \mathcal{D}\left(t, u_{t}\right)+\int_{0}^{t} B(t-s) \mathcal{D}\left(s, u_{s}\right) d s+g\left(t, u_{t}\right), \quad t \in(0, a), \\
u_{0} & =\varphi \in \mathcal{B},
\end{aligned}\right.
$$

onde $\mathcal{D}(t, \psi)=\psi(0)+f(t, \psi)$ e $f, g:[0, a] \times X \rightarrow X$ são funções apropriadas. Usando diferentes resultados de ponto fixo, nos Teoremas 2.1.1, 2.1.2 e 2.1.3, é estabelecida a existência de soluções fracas para (9). Por outro lado, nos Teoremas 2.2.1 e 2.2.2 são estabelecidas condições às quais uma solução fraca é uma solução semi-clássica e clássica respectivamente. Como aplicação dos resultados do Capítulo 2, no Exemplo 2.3 estudamos um sistema abstrato que permite a análise do sistema integro-diferencial (5) no caso particular onde $K_{1}=K_{2}$. 
O Capítulo 3 é dedicado ao estudo do sistema integro-diferencial

$$
\left\{\begin{aligned}
\frac{d}{d t}\left(x(t)+F\left(t, x_{t}\right)\right) & =A x(t)+\int_{0}^{t} B(t-s) x(s) d s+G\left(t, x_{t}\right), \quad t \in(0, a), \\
x_{0} & =\varphi \in \mathcal{B},
\end{aligned}\right.
$$

onde $A: D(A) \subset X \rightarrow X, B(t): D(B(t)) \subset X \rightarrow X, t \geq 0$ são operadores fechados em $X$, a história $x_{t}:(-\infty, 0] \rightarrow X, x_{t}(\theta)=x(t+\theta)$ pertence a um espaço de fase abstrato $\mathcal{B}$ definido axiomaticamente e $F, G:[0, a] \times \mathcal{B} \rightarrow X$ são funções apropriadas.

Como é observado no Capítulo 3, o tratamento deste sistema é diferente e de fato mais complicado que aquele usado no estudo de (9). Para obter nossos resultados de existência usamos propriedades e conceitos relacionados com a teoria de Resolventes analíticos, veja como referência [25].

Em geral os resultados de existência do Capítulo 3 são obtidos usando Teoremas de ponto fixo. Especificamente, no Teorema 3.1.1 é provado a existência de soluções fracas usando o critério da contração. Por outro lado, os Teoremas 3.1.2 e 3.1.3 estabelecem a existência de soluções fracas por meio dos Teoremas de Schauder e Leray-Schauder, respectivamente. Neste Capítulo também consideramos a existência de soluções semi-clássicas, veja Teorema 3.2.1 e clássicas, veja Teorema 3.2.3, para 10). O Capítulo é completado com uma aplicação dos resultados abstratos.

No Capítulo 4 estudamos a existência e regularidade de soluções para uma classe de sistemas neutros integro-diferenciais descritos da forma

$$
\left\{\begin{aligned}
\frac{d}{d t}\left(x(t)+\int_{-\infty}^{t} N(t-s) x(s) d s\right) & =A x(t)+\int_{0}^{t} B(t-s) x(s) d s+G\left(t, x_{t}\right), \quad t>0 \\
x_{0} & =\varphi \in \mathcal{B}
\end{aligned}\right.
$$

onde $A: D(A) \subset X \rightarrow X, B(t): D(B(t)) \subset X \rightarrow X, t \geq 0$ são operadores lineares fechados com $D(B(t)) \supset D(A), t \geq 0$ e $(N(t))_{t \geq 0}$ é uma família de operadores lineares contínuos em $\mathrm{X}$.

Para obter nossos resultados de existência de soluções, na primeira parte do Capítulo 4 desenvolvemos uma teoria de operadores resolventes para o sistema integro-diferencial

$$
\left\{\begin{aligned}
\frac{d}{d t}\left(x(t)+\int_{0}^{t} N(t-s) x(s) d s\right) & =A x(t)+\int_{0}^{t} B(t-s) x(s) d s, \quad t \in(0, a), \\
x(0) & =x_{0} .
\end{aligned}\right.
$$

à qual denominaremos família "N-resolvente". Dizemos que uma família de operadores lineares fortemente contínua $(R(t))_{t \geq 0}$ em $X$ é uma família N-resolvente para $(12)$ se $R(0)=$ 
$I_{d}$ e as equações

$$
\begin{aligned}
\frac{d}{d t}\left(R(t) x+\int_{0}^{t} N(t-s) R(s) x d s\right) & =A R(t) x+\int_{0}^{t} B(t-s) R(s) x d s, \\
\frac{d}{d t}\left(R(t) x+\int_{0}^{t} R(t-s) N(s) x d s\right) & =R(t) A x+\int_{0}^{t} R(t-s) B(s) x d s,
\end{aligned}
$$

são satisfeitas para todo $t \geq 0$.

Os resultados básicos deste Capítulo são os Teorema 4.2.1 onde é provado a existência de uma família N-resolvente analítica para (12), e o Teorema 4.3.1 onde é introduzida uma forma da variação dos parâmetros para 12 . Finalmente, no Teorema 4.4 .3 aplicamos a teoria de operadores N-resolvente para o estudo da existência de soluções clássicas para o sistema (11).

As notações presentes nesta Tese são aquelas usadas em Análise Funcional. Em particular, para espaços normados $\left(Y,\|\cdot\|_{Y}\right)$ e $\left(Z,\|\cdot\|_{Z}\right), \mathcal{L}(Y, Z)$ representa o espaço dos operadores lineares contínuos de $Y$ em $Z$ munido da norma uniforme de operadores. A notação $\mathcal{L}(Y)$ e reservada para $\mathcal{L}(Y, Y)$. Para $x \in Z$ e $r>0, B_{r}(x, Z)$ representa a bola de centro $x$ e raio $r$ em $Z$. 


\title{
Capítulo 1
}

\section{Preliminares}

\begin{abstract}
Resumo
Neste capítulo introduzimos definições, conceitos e propriedades básicas da teoria de semigrupos de operadores lineares, operadores resolventes para equações integro-diferenciais e espaço de fase abstrato que serão usados durante o decorrer do trabalho.
\end{abstract}

\subsection{Semigrupos de operadores lineares}

Introduzimos a seguir, conceitos e propriedades básicas da teoria de semigrupos de operadores lineares limitados em espaços de Banach. No que segue, $X$ é um espaço de Banach e $\mathcal{L}(X)$ é o espaço dos operadores lineares contínuos de $X$ em $X$ munido da norma uniforme de operadores.

Definição 1.1.1 Uma família de operadores $(T(t))_{t \geq 0}$ em $\mathcal{L}(X)$ é um semigrupo de operadores se

(a) $T(0)=I_{d}$

(b) $T(t+s)=T(t) T(s), \quad t, s \geq 0$.

Definição 1.1.2 Um semigrupo de operadores lineares $(T(t))_{t \geq 0}$ é chamado de $C_{0}$-semigrupo se para todo $x \in X$, a função $t \rightarrow T(t) x$ é contínua no zero. O semigrupo é chamado uniformemente contínuo se a função $[0, \infty) \rightarrow \mathcal{L}(X) ; t \rightarrow T(t)$ é contínua no zero. 
Definição 1.1.3 Sejam $(T(t))_{t \geq 0}$ um $C_{0}$-semigrupo de operadores lineares em $X$ e $D(A) o$ conjunto

$$
D(A)=\left\{x \in X: \lim _{t \downarrow 0} \frac{T(t) x-x}{t} \text { existe }\right\} .
$$

$O$ operador $A: D(A) \subset X \rightarrow X$ definido por

$$
A x=\lim _{t \downarrow 0} \frac{T(t) x-x}{t}=\left.\frac{d^{+}}{d t} T(t) x\right|_{t=0}, \quad x \in D(A),
$$

é chamado o gerador infinitesimal do semigrupo $(T(t))_{t \geq 0}$.

No próximo resultado, resumiremos algumas propriedades básicas relativas a $C_{0}$-semigrupos.

Teorema 1.1.1 [63] Sejam $(T(t))_{t \geq 0}$ um $C_{0}$-semigrupo de operadores lineares em $X$ e A seu gerador infinitesimal. Então as seguintes propriedades são verificadas.

(a) O semigrupo $(T(t))_{t \geq 0}$ é uniformemente contínuo, se e somente se, A é um operador limitado.

(b) Existem constantes $\omega \geq 0$ e $M>1$ tais que $\|T(t)\| \leq M e^{\omega t}$, para todo $t \geq 0$.

(c) Para todo $t>0$ e todo $x \in X, \lim _{s \rightarrow t} T(s) x=T(t) x$. Mais ainda, se o semigrupo é uniformemente contínuo então $\lim _{s \rightarrow t} T(s)=T(t)$ em $\mathcal{L}(X)$.

(d) Para todo $x \in X$ e todo $t \geq 0, \lim _{h \downarrow 0} \frac{1}{h} \int_{t}^{t+h} T(s) x d s=T(t) x$.

(e) Para todo $x \in X$ e todo $t>\tau \geq 0, \quad \int_{\tau}^{t} T(s) x d s \in D(A)$ e $A \int_{\tau}^{t} T(s) x d s=T(t) x-$ $T(\tau) x$.

(f) Para todo $x \in D(A)$ e todo $t \geq 0, T(t) x \in D(A)$ e $\frac{d}{d t} T(t) x=A T(t) x=T(t) A x$.

(g) A é linear, fechado e $\overline{D(A)}=X$.

Apresentamos a seguir o famoso Teorema de Hille Yosida. Este Teorema estabelece condições necessárias e suficientes para que um operador linear fechado seja o gerador infinitesimal de um $C_{0}$-semigrupo de contrações em $X$. Previamente consideremos algumas definições.

Definição 1.1.4 Seja $(T(t))_{t \geq 0}$ um $C_{0}$-semigrupo operadores lineares em $X$. Diremos $(T(t))_{t \geq 0}$ é um $C_{0}$-semigrupo de contrações se $\|T(t)\| \leq 1$ para todo $t \geq 0$. 
Definição 1.1.5 Seja $A: D(A) \subset X \rightarrow X$ um operador linear. O conjunto resolvente de $A$, denotado por $\rho(A)$, é definido por

$$
\rho(A)=\left\{\lambda \in \mathbb{C} ;(\lambda I-A) \text { é inversível e }(\lambda I-A)^{-1} \in \mathcal{L}(X)\right\} .
$$

A função de $\rho(A)$ em $\mathcal{L}(X)$, definida por $\lambda \mapsto R(\lambda: A)=(\lambda I-A)^{-1}$, é chamada resolvente de A.

Teorema 1.1.2 (Hille-Yosida)[63, Theorem 3.1] Um operador linear $A: D(A) \subset X \rightarrow X$ é o gerador infinitesimal de um $C_{0}$-semigrupo de contrações se, e somente se,

(i) A é fechado e $\overline{D(A)}=X$;

(ii) $\rho(A) \supset(0, \infty) e\|R(\lambda: A)\| \leq \frac{1}{\lambda}$, para todo $\lambda>0$.

A seguir resumiremos e comentaremos alguns fatos básicos a respeito de semigrupos compactos e analíticos. Incluímos também de forma sucinta, algumas propriedades das potências fracionárias associadas com o gerador de um semigrupo analítico.

Definição 1.1.6 Um $C_{0}$-semigrupo, $(T(t))_{t \geq 0}$, de operadores lineares é compacto para $t>t_{0}$, se para todo $t>t_{0}$ o operador $T(t)$ é compacto. O semigrupo $(T(t))_{t \geq 0}$ é chamado compacto, se é compacto para todo $t>0$.

Notemos que se $X$ é um espaço de dimensão finita então todo $C_{0}$-semigrupo de operadores lineares é compacto. Mais ainda, se um $C_{0}$-semigrupo $(T(t))_{t \geq 0}$ é um $C_{0}$-semigrupo compacto para $t \geq 0$ então $X$ é de dimensão finita.

Teorema 1.1.3 [63, Theorem 3.2] Seja $(T(t))_{t \geq 0}$ um $C_{0}$-semigrupo de operadores lineares em $X$. Se $T(t)$ é compacto para $t>t_{0}$, então a função $\left(t_{0}, \infty\right) \rightarrow \mathcal{L}(X) ; t \rightarrow T(t)$ é contínua.

O próximo Teorema caracteriza quando um $C_{0}$-semigrupo é compacto.

Teorema 1.1.4 [63, Theorem 3.3] Seja $(T(t))_{t \geq 0}$ um $C_{0}$-semigrupo de operadores lineares limitados em $X$ e A seu gerador infinitesimal. O semigrupo $(T(t))_{t \geq 0}$ é compacto, se e somente se, a função $(0, \infty) \rightarrow \mathcal{L}(X) ; t \rightarrow T(t)$ é contínua e $R(\lambda: A)$ é compacto para todo $\lambda \in \rho(A)$.

Nossos resultados de existência, em alguns casos, são especialmente aplicados para o caso em que $A$ é o gerador de um semigrupo analítico. Por isto, introduzimos agora elementos básicos associados a este tipo de semigrupo. 
Definição 1.1.7 Sejam $0<\phi_{1}<\phi_{2}$ e $\Delta=\left\{z \in \mathbb{C}: \phi_{1} \leq \arg (z) \leq \phi_{2}\right\}$. Uma família de operadores lineares limitados $(T(z))_{z \in \Delta}$ em $X$ é um semigrupo analítico em $\Delta$, se as seguintes condições são verificadas.

(i) A função $z \rightarrow T(z)$ é analítica em $\Delta$;

(ii) $T(0)=I_{d}$ e $\lim _{z \rightarrow 0} T(z) x=x$ para todo $x \in X$;

(iii) $T\left(z_{1}+z_{2}\right)=T\left(z_{1}\right) T\left(z_{2}\right)$ para todo $z_{1}, z_{2} \in \Delta$.

O seguinte resultado é um clássico Teorema de caracterização de semigrupos analíticos.

Teorema 1.1.5 [63, Theorem 5.2] Seja $(T(t))_{t \geq 0}$ um $C_{0}$-semigrupo uniformemente limitado de operadores lineares, $A$ seu gerador infinitesimal e suponha que $0 \in \rho(A)$. Então as seguintes afirmações são equivalentes:

(a) O semigrupo $(T(t))_{t \geq 0}$ pode ser estendido a um semigrupo analítico em um setor $\Delta_{\delta}=$ $\{z:|\arg (z)|<\delta\}, \delta>0$, tal que $(T(z))_{z \in \Delta_{\delta}}$ é uniformemente limitado em cada sub-setor $\Delta_{\delta^{\prime}}$, com $\delta^{\prime}<\delta$.

(b) Existe uma constante $C>0$ tal que para cada $\sigma>0$ e todo $\tau \neq 0\|R(\sigma+i \tau: A)\| \leq \frac{C}{|\tau|}$.

(c) Existe $0<\delta<\frac{\pi}{2}$ e $M>0$ tal que

$$
\rho(A) \supset \Sigma^{+}=\{\lambda \in \mathbb{C}: 0<\omega<|\arg (\lambda)| \leq \pi\} \cup\{0\}
$$

$e$

$$
\|R(\lambda: A)\| \leq \frac{M}{|\lambda|}, \quad \lambda \in \Sigma, \quad \lambda \neq 0
$$

(d) A função $t \rightarrow T(t)$ é diferenciável em $(0, \infty)$ e existe $C>0$ tal que

$$
\|A T(t)\| \leq \frac{C}{t}, \quad t>0
$$

No que resta deste capítulo assumiremos que $(T(t))_{t \geq 0}$ é um $C_{0}$-semigrupo de operadores lineares e $A$ é seu gerador infinitesimal. Para estabelecermos o conceito de potência fracionária de $A$ introduzimos a seguinte condição. 
(H) Existem constantes $\omega$ e $M$ positivas tais que

$$
\rho(A) \supset \Sigma^{+}=\{\lambda \in \mathbb{C}: 0<\omega<|\arg (\lambda)| \leq \pi\} \cup V
$$

e

$$
\|R(\lambda: A)\| \leq \frac{M}{1+|\lambda|}, \lambda \in \Sigma^{+},
$$

onde $V$ é uma vizinhança do zero

Definição 1.1.8 Assuma que A verifica a condição $(\mathbf{H})$. Para $\alpha>0$ definimos

$$
A^{-\alpha}=\frac{1}{2 \pi i} \int_{C_{r}} z^{-\alpha}(A-z I)^{-1} d z
$$

onde $C_{r}=C_{1} \cup C_{2} \cup C_{3} \subset \Sigma^{+}$é uma curva dada por

$$
\begin{aligned}
& C_{1}=\left\{\rho e^{i v}: \rho \geq r\right\}, \\
& C_{2}=\left\{r e^{i \theta}:-v \leq \theta \leq v\right\}, \\
& C_{3}=\left\{-\rho e^{-i v}: \rho \geq r\right\},
\end{aligned}
$$

sendo $\omega<v<\pi$ e $r>0$.

Teorema 1.1.6 [63, Lemmas 6.2-6.6] As seguintes propriedades são válidas.

(a) Se $\alpha, \beta \geq 0$ então $A^{-(\alpha+\beta)}=A^{-\alpha} A^{-\beta}$.

(b) O conjunto $\left\{A^{-\alpha}: \alpha \in(0,1)\right\}$ é limitado em $\mathcal{L}(X)$.

(c) $\lim _{\alpha \downarrow 0} A^{-\alpha} x=x$ para todo $x \in X$.

(d) $A^{-\alpha}$ é injetora para cada $\alpha \geq 0$.

Definição 1.1.9 Suponha que A satisfaz a condição $(\mathbf{H})$ com $\omega<\frac{\pi}{2}$. Para cada $\alpha>0$ definimos $A^{\alpha}=\left(A^{-\alpha}\right)^{-1}$.

Teorema 1.1.7 [63, Theorem 6.8] Seja $A^{\alpha}$ como na Definição 1.1.9. As seguintes propriedades são válidas.

(a) $A^{\alpha}$ é um operador fechado com $D\left(A^{\alpha}\right)=\operatorname{Im}\left(A^{-\alpha}\right)$.

(b) Para $\alpha \geq \beta>0$ temos $D\left(A^{\alpha}\right) \subset D\left(A^{\beta}\right)$.

(c) $\overline{D\left(A^{\alpha}\right)}=X$ para cada $\alpha \geq 0$. 
(c) $A^{\alpha+\beta} x=A^{\alpha} A^{\beta} x$ para cada $x \in D\left(A^{\gamma}\right)$ onde $\gamma=\max \{\alpha, \beta, \alpha+\beta\}$.

Teorema 1.1.8 [63, Theorem 6.13] Seja $-A$ o gerador infinitesimal de um semigrupo analítico $T(t)$ e $0<\gamma \leq \eta \leq 1$. Se $0 \in \rho(A)$ e $X_{\alpha}$ é o espaço $D\left(A^{\alpha}\right)$ munido da norma do gráfico, então as seguintes propriedades são verificadas.

(a) $X_{\eta}$ é um espaço de Banach, $X_{\eta} \hookrightarrow X_{\gamma}$.

(b) Para cada $\eta>0$, a função $s \rightarrow(-A)^{\eta} T(s)$ é contínua sobre $(0, \infty)$ na topologia uniforme de operadores, e existe $C_{\eta}>0$ tal que $\left\|(-A)^{\eta} T(t)\right\| \leq \frac{C_{\eta}}{t^{\eta}}$, para todo $t>0$.

(c) Para todo $t>0$ e todo $\alpha>0, T(t)(X) \subset D\left(A^{\alpha}\right)$. Mais ainda, para $t \geq 0$ e $x \in D\left(A^{\alpha}\right)$. temos que $T(t) A^{\alpha} x=A^{\alpha} T(t) x$.

(d) Para cada $\alpha \in(0,1]$ existe $C_{\alpha}>0$ tal que $\|T(t) x-x\| \leq C_{\alpha} t^{\alpha}\left\|A^{\alpha} x\right\|$, para todo $x \in D\left(A^{\alpha}\right)$ e todo $t>0$.

\subsection{Operador resolvente para equações integro-diferenciais}

Nosso estudo de existência de soluções para sistemas neutros do tipo integro-diferencial será feito fazendo uso da teoria de operadores resolventes. Brevemente, um operador resolvente de operadores lineares em $X$ é uma família a um parâmetro de operadores lineares em $X$, que se relaciona com o sistema integro-diferencial

$$
\begin{aligned}
& x^{\prime}(t)=A x(t)+\int_{0}^{t} B(t-s) x(s) d s, \quad t>0, \\
& x(0)=x_{0} \in X
\end{aligned}
$$

de maneira similar como semigrupo de operadores lineares se relaciona com a equação linear $x^{\prime}(t)=A x(t)$.

Em relação a teoria de operadores resolventes associados a sistemas integro-diferenciais citamos, [26] para existência de operadores resolvente; [57] para o estudo de existência e regularidade das soluções para sistemas integro-diferencias semi-lineares e 25] para resolvente analíticos entre outros.

Considere o sistema integro-diferencial

$$
\begin{aligned}
& x^{\prime}(t)=A x(t)+\int_{0}^{t} B(t-s) x(s) d s, \quad t \in(0, a), \\
& x(0)=x_{0} \in X .
\end{aligned}
$$


Nesta tese sempre assumiremos que $A: D(A) \subset X \rightarrow X$ é um operador fechado densamente definido em $X$; que cada $B(t): D(B(t)) \subset X \rightarrow X$ é um operador linear fechado em $X$ com $D(B(t)) \supset D(A)$ e que a seguinte hipótese é verificada.

$\left(\mathbf{H}_{\mathbf{0}}\right) \quad A: D(A) \subset X \rightarrow X$ é um operador fechado densamente definido em $X$; que cada $B(t): D(B(t)) \subset X \rightarrow X$ é um operador linear fechado em $X \operatorname{com} D(B(t)) \supset D(A)$, para todo $x \in D(A)$ a função $t \rightarrow B(t) x$ e fortemente mensuráveis em $(0, \infty)$ e existe $b \in L_{l o c}^{1}\left(\mathbb{R}^{+}\right)$e $\beta \in \mathbb{R}^{+}$com $b(t) e^{-\beta t} \in L^{1}\left(\mathbb{R}^{+}\right)$tal que $\|B(t) x\| \leq b(t)(\|x\|+\|A x\|)$ para todo $x \in D(A)$.

Definição 1.2.1 Uma familia de operadores lineares $(R(t))_{t \geq 0}$ em $\mathcal{L}(X)$ é chamada operador resolvente associada a (1.1)-(1.2) se as seguintes propriedades são verificadas:

(a) $R(0)=I_{d}$, a função $t \rightarrow R(t) x$ é contínua sobre $[0, \infty)$ para todo $x \in X$ e $R(t) D(A) \subset$ $D(A)$ para todo $t>0$;

(b) Se $x \in D(A), t \rightarrow A R(t) x$ é contínua sobre $[0, \infty)$ e a função $t \rightarrow R(t) x$ é continuamente diferenciável sobre $[0, \infty)$ e para todo $t \geq 0$ e

$$
\begin{aligned}
& R^{\prime}(t) x=A R(t) x+\int_{0}^{t} B(t-\xi) R(\xi) x d \xi, \\
& R^{\prime}(t) x=R(t) A x+\int_{0}^{t} R(t-\xi) B(\xi) x d \xi .
\end{aligned}
$$

Apresentamos agora algumas propriedades básicas da teoria de operadores resolvente que serão usadas em nosso trabalho.

Lema 1.2.1 [26, Lemma 1] Seja $(R(t))_{t \geq 0}$ uma família resolvente associada a 1.1)-1.2). Então as seguintes propriedades são válidas.

(1) A família resolvente é $(R(t))_{t \geq 0}$ é única.

(2) Se $U(t), t \geq 0$, é o operador definido por $U(t)=\int_{0}^{t} R(s) d s$, então $U(t) X \subset D(A)$.

Definição 1.2.2 [26, Definition 2] O sistema [1.1]-11.2] é chamado de bem posto se as seguintes condições são verificadas.

(a) Para cada $x \in D(A)$ existe uma única solução $u(\cdot, x)$ de (1.1)-1.2).

(b) Se $\left(x_{n}\right)_{n \in \mathbb{N}}$ é uma seqüência em $D(A)$ tal que $x_{n} \rightarrow 0$ quando $n \rightarrow \infty$ então $u\left(t, x_{n}\right) \rightarrow 0$ quando $n \rightarrow \infty$ uniformemente em intervalos limitados de $[0, \infty)$. 
A relação entre a existência de família resolvente para (1.1)-(1.2) e do sistema ser bem posto é estabelecida no seguinte resultado.

Teorema 1.2.1 [26, Theorem 4] A equação 1.1)-11.2] admite um operador resolvente, se $e$ somente se, o sistema (1.1)-1.2) é bem posto.

Como no caso de semigrupos lineares, existem resultados de geração do tipo Hille-Yosida para operadores resolvente, veja por exemplo, Grimmer \& Prüss [26] e Sforza [66]. No próximo resultado, $\widehat{B}(\lambda)$ é a transformada de Laplace da funçaõ $t \rightarrow B(t)$.

Definição 1.2.3 Uma família resolvente $(R(t))_{t \geq 0}$ associada a (1.1)-(1.2) é dita exponencialmente limitada se existem constantes $\omega_{0}$ e $M \geq 1$ tais que $\|R(t)\| \leq M e^{\omega_{0} t}$ para todo $t \geq 0$.

Teorema 1.2.2 [26, Theorem 8] Assuma que $\left(\mathbf{H}_{\mathbf{0}}\right)$ é verificada. Então existe um operador resolvente exponencialmente limitado $(R(t))_{t \geq 0}$ associado a (1.1)-(1.2), se e somente se, as seguintes condições são verificadas.

(a) Existe $\omega>\beta$ e $M \geq 1$ tal que $(\lambda-A-\widehat{B}(\lambda))$ é fechado, com domínio $D(A)$;

(b) O operador $(\lambda-A-\widehat{B}(\lambda))$ é invertivel para $\operatorname{Re}(\lambda)>\omega$; a função $\lambda \rightarrow H(\lambda)=$ $(\lambda-A-\widehat{B}(\lambda))^{-1}$ é analítica e holomorfa sobre $\mathcal{L}(X) e$

$$
\left\|\frac{1}{n !} \frac{d^{n}}{d \lambda^{n}} H(\lambda)\right\| \leq \frac{M}{(\operatorname{Re}(\lambda)-\omega)^{n+1}}, \quad \operatorname{Re}(\lambda)>\omega, n \in \mathbb{N}_{0}=\mathbb{N} \cup\{0\} .
$$

Observação 1.2.1 Se $(R(t))_{t \geq 0}$ é uma família resolvente exponencialmente limitada associado a 1.1-1.2) e $B(t)=0$ para todo $t \in \mathbb{R}^{+}$, então $(R(t))_{t \geq 0}$ é um $C_{0}$-semigrupo em $X$.

Teorema 1.2.3 [26, Theorem 6] Seja $(R(t))_{t \geq 0}$ uma familia resolvente associada 1.1)-(1.2) exponencialmente limitada. Então

$$
D(A)=\left\{x \in X: \lim _{h \downarrow 0} \frac{R(h) x-x}{h} \text { existe }\right\}
$$

$e$

$$
A x=\lim _{h \downarrow 0} \frac{R(h) x-x}{h}, \quad x \in D(A) .
$$


Para continuar incluímos alguns resultados relacionados com o problema não homogêneo

$$
\begin{aligned}
& x^{\prime}(t)=A x(t)+\int_{0}^{t} B(t-s) x(s) d s+f(t), \quad t \in(0, a), \\
& x(0)=x_{0} \in X .
\end{aligned}
$$

No que segue desta seção assumiremos que $(R(t))_{t \geq 0}$ é uma família resolvente para (1.1)- 1.2 .

Definição 1.2.4 Uma função $u:[0, a] \rightarrow X$ é chamada solução clássica de (1.3)-1.4), se $u(\cdot)$ é continuamente diferenciável em $(0, a), u(t) \in C((0, a):[D(A)])$ e (1.3)-(1.4) é verificada em $[0, a)$.

Teorema 1.2.4 [26, Theorem 2] Seja $x_{0} \in X$ e $f \in C([0, a]: X)$. Se u(·) é uma solução clássica de (1.3)-1.4 então

$$
u(t)=R(t) x_{0}+\int_{0}^{t} R(t-s) f(s) d s, \quad \forall t \in[0, a] .
$$

Como no caso de semigrupos lineares, a função $u(\cdot)$ definida por 1.5 não é em geral solução clássica de (1.3)-1.4). Por isso introduzimos os seguintes conceitos.

Definição 1.2.5 Seja $f \in L^{1}([0, a]: X)$. Dizemos que uma função $u \in C([0, a]: X)$ é uma solução fraca de (1.3)-(1.4) se

$$
u(t)=R(t) x+\int_{0}^{t} R(t-s) f(s) d s, \quad \forall t \in[0, a] .
$$

Os próximos resultados estabelecem condições básicas nas quais uma solução fraca é clássica. No que segue $[D(A)]$ é o espaço $D(A)$ munido da norma do gráfico.

Teorema 1.2.5 [26, Theorem 8] Seja $u_{0} \in D(A)$. Se a função $f \in L^{1}([0, a]:[D(A)])$ ou $f \in W^{1,1}([0, a]: X)$ então a função $u(\cdot)$ definida por 1.5) é uma solução clássica de (1.3)- (1.4).

Corolário 1.2.1 [26, Corollary 2] Assuma que $f \in C([0, a]: X)$. A função definida por $u(t)=\int_{0}^{t} R(t-s) f(s) d s$ é solução de 1.3$)$-1.4 com $u_{0}=0$, se uma das seguintes condições são satisfeitas

(i) $u \in C^{1}([0, a]: X)$;

(ii) $u \in C([0, a]:[D(A)])$.

Se u é uma solução clássica sobre [0, a] então u satisfaz as condições (i) e (ii). 


\subsection{Espaços de fase abstratos e alguns Teoremas}

Neste trabalho, o espaço de fase $\mathcal{B}$ é definido de maneira axiomática. Especificamente, $\mathcal{B}$ será um espaço vetorial formado por funções definidas de $(-\infty, 0]$ em $X$, munido de uma seminorma $\|\cdot\|_{\mathcal{B}}$ e tal que as seguintes propriedades são verificadas.

(A) Se $x:(-\infty, \sigma+b) \rightarrow X, b>0$, é contínua sobre $[\sigma, \sigma+b)$ e $x_{\sigma} \in \mathcal{B}$, onde $x_{\sigma}(\theta):=$ $x(\sigma+\theta)$, então para cada $t \in[\sigma, \sigma+b)$, as seguintes propriedades são válidas:

(i) $x_{t} \in \mathcal{B}$,

(ii) $\|x(t)\| \leq H\left\|x_{t}\right\|_{\mathcal{B}}$,

(iii) $\left\|x_{t}\right\|_{\mathcal{B}} \leq K(t-\sigma) \sup \{\|x(s)\|: \sigma \leq s \leq t\}+M(t-\sigma)\left\|x_{\sigma}\right\|_{\mathcal{B}}$,

sendo $H$ uma constante positiva, $K, M:[0, \infty) \rightarrow[0, \infty), K(\cdot)$ contínua, $M(\cdot)$ localmente limitada e onde $H, K$ e $M$ são independentes de $x(\cdot)$.

(A1) Para a função $x(\cdot)$ em $(\mathbf{A})$, a função $t \rightarrow x_{t}$ é uma função contínua de $[\sigma, \sigma+b)$ em $\mathcal{B}$.

(B) O espaço $\mathcal{B}$ é completo.

Para $t \geq 0$ definimos $S(t), W(t): \mathcal{B} \rightarrow \mathcal{B}$ por

$$
\begin{gathered}
{[S(t) \varphi](\theta)=\left\{\begin{array}{r}
\varphi(0) \text { se }-t \leq \theta \leq 0, \\
\varphi(t+\theta) \text { se }-\infty<\theta<-t,
\end{array}\right.} \\
{[W(t) \varphi](\theta)=\left\{\begin{aligned}
R(t+\theta) \varphi(0) & \text { se }-t \leq \theta \leq 0, \\
\varphi(t+\theta) & \text { se }-\infty<\theta<-t .
\end{aligned}\right.}
\end{gathered}
$$

Dos axiomas do espaço $\mathcal{B}$ é fácil deduzir que $(S(t))_{t \geq 0}$ é um semigrupo de classe $C_{0}$ sobre $\mathcal{B}$.

Para obter alguns dos nossos resultados, precisaremos de algumas propriedades adicionais para o espaço de fase $\mathcal{B}$.

(C2) Se $\left(\varphi_{n}\right)_{n \in \mathbb{N}}$ é uma seqüência uniformemente limitada em $\left.C_{00}(X)\right)$ e assuma que $\varphi_{n} \rightarrow \varphi$ uniformemente em compactos. Então $\varphi \in \mathcal{B}$ e $\left\|\varphi_{n}-\varphi\right\|_{\mathcal{B}} \rightarrow 0$, quando $n \rightarrow \infty$.

(C3) Seja $x:(-\infty, \sigma+b] \rightarrow X$ contínua, $\operatorname{com} x_{\sigma}=0$ e tal que derivada a direita $x^{\prime}\left(\sigma^{+}\right)$existe. Seja $\psi:(-\infty, 0] \rightarrow X$ a função definida por $\psi(\theta)=0$ para $\theta<0$ e $\psi(0)=x^{\prime}\left(\sigma^{+}\right)$. Se $\psi \in \mathcal{B}$ então $\left\|\frac{1}{h} x_{\sigma+h}-\psi\right\|_{\mathcal{B}} \rightarrow 0$ quando $h \downarrow 0$. 
Para detalhes relacionados com os axiomas $(\mathbf{C 2}),(\mathbf{C 3})$ veja [33, 49]. Como complemento, apresentamos alguns exemplo de espaços de fases.

\section{Exemplo 1.3.1 O espaço $C_{g}(\cdot)$.}

Seja $g:(-\infty, 0] \rightarrow[0, \infty)$ uma função positiva, contínua, com $g(0)=1$, e tal que as seguintes condições são verificadas.

(g-1) A função $\gamma(t):=\sup _{-\infty<\theta \leq-t} \frac{g(t+\theta)}{g(\theta)}$ é localmente limitada para $t \geq 0$.

(g-2) $g(\theta) \rightarrow \infty$ quando $\theta \rightarrow-\infty$

Definimos por $C_{g}^{0}$ o espaço formado pelas funções contínuas $\varphi:(-\infty, 0] \rightarrow X$ tais que $\frac{\|\varphi(\theta)\|}{g(\theta)} \rightarrow 0$ quando $\theta \rightarrow-\infty$. Consideraremos $C_{g}^{0}$ munido com a norma

$$
\|\varphi\|_{g}:=\sup _{-\infty<\theta \leq-t} \frac{\|\varphi(\theta)\|}{g(\theta)}
$$

Nessas condições, o par $\left(C_{g}^{0},\|\cdot\|_{g}\right)$ é um espaço de fase que verifica os axiomas $\mathbf{A}, \mathbf{A}_{\mathbf{1}}$ e $\mathbf{B}$.

Exemplo 1.3.2 O espaço $C_{r} \times L^{2}(\rho, X)$.

Seja $\rho:[0, \infty) \rightarrow \mathbb{R}$ positiva, contínua, decrescente, com $\rho(0)=1$ e tal que $\rho(t) \rightarrow 0$ quando $t \rightarrow \infty$. Seja $\mathcal{B}=C_{r} \times L^{2}(\rho, X)$ o espaço formado pelas funções $\varphi:(-\infty, 0] \rightarrow X$ tais que $\rho\|\varphi\|^{2}$ é Lebesgue integrável sobre $(-\infty, r]$ e $\left.\varphi\right|_{[-r, 0]} \in C([-r, 0] ; X)$. Sobre o espaço $C_{r} \times L^{2}(\rho, X)$ consideramos a semi-norma $\|\cdot\|_{\mathcal{B}}$ definida por

$$
\|\varphi\|_{\mathcal{B}}:=\left(\int_{-\infty}^{-r} \rho(\theta)\|\varphi(\theta)\|^{2} d \theta\right)^{1 / 2}+\sup _{\theta \in[-r, 0]}\|\varphi(\theta)\| .
$$

Assumamos adicionalmente que existe uma função integrável e localmente limitada $\gamma:(-\infty,-r] \rightarrow[1, \infty)$ tal que $\rho(\xi+\theta) \leq \gamma(\xi) \rho(\theta)$, para todo $\xi \leq 0$ e todo $\theta \in(-\infty,-r] \backslash N_{\xi}$ sendo $N_{\xi} \subseteq(-\infty,-r]$ um conjunto de medida zero. Nessas condições, o par $\left(\mathcal{B},\|\cdot\|_{\mathcal{B}}\right)$ é um espaço de fase que verifica os axiomas $\mathbf{A}, \mathbf{A}_{\mathbf{1}} e \mathbf{B}$. Mais ainda, no caso $r=0, p=2$ temos que $M(t)=\gamma(-t)^{\frac{1}{2}}$ e que $K(t)=1+\left(\int_{-t}^{0} \rho(\tau) d \tau\right)^{\frac{1}{2}}$ para todo $t \geq 0$.

Nossos resultados sobre existência de soluções são obtidos usando Teoremas de ponto fixo. Assim para facilitar a leitura deste trabalho considere os seguintes resultados.

Teorema 1.3.1 [59, Corollary 4.3.2] Seja D um subconjunto convexo, limitado e fechado de um espaço de Banach $Z$. Se $B, C: D \rightarrow Z$ são funções contínuas tais que: 
(a) $B z+C z \in D$ para todo $z \in Z$;

(b) $\overline{C(D)}$ é compacto;

(c) Existe $0 \leq \gamma<1$ tal que $\|B z-B w\| \leq \gamma\|z-w\|$ para todo $z, w \in D$, então existe $u \in D$ tal que $B u+C u=u$.

Observação 1.3.1 Se um operador $L: D \rightarrow C$, pode ser escrito da forma $L=B+C$, onde $B, C$ estão nas condições do Teorema 1.3.1, então L é dito operador condensante.

Teorema 1.3.2 [23, Theorem 6.5.4] (Leray Schauder Alternative) Seja D um subconjunto convexo fechado de um espaço de Banach $Z$ e assuma que $0 \in D$. Se $F: D \rightarrow D$ uma aplicação completamente contínua, então o conjunto $\{x \in D: x=\lambda F(x), 0<\lambda<1\}$ é não limitado ou $F$ possui um ponto fixo em $D$.

Para finalizar este capítulo, consideramos o seguinte critério de valor médio para a integral de Bochner o qual será usado frequentemente neste trabalho. No que segue deste trabalho $\operatorname{co}\{A\}$ representa a envoltória convexa do conjunto $A$.

Teorema 1.3.3 [59, Lemma 1.3] Seja $Z$ um espaço de Banach e $f:[\alpha, \beta] \rightarrow Z$ uma função integrável. Então

$$
\frac{1}{\beta-\alpha} \int_{\alpha}^{\beta} f(\tau) d \tau \in \overline{c o}\{f(\tau): \tau \in[\alpha, \beta]\} .
$$




\title{
Capítulo 2
}

\section{Existência e regularidade de}

\section{soluções para uma equação}

\section{integro-diferencial do tipo neutra}

\begin{abstract}
Resumo
Neste capítulo usaremos a teoria de operadores resolventes para estudar a existência de soluções fracas, semi-clássicas e clássicas para o sistema integro-diferencial do tipo neutro

$$
\begin{aligned}
\frac{d}{d t} \mathcal{D}\left(t, x_{t}\right) & =A \mathcal{D}\left(t, x_{t}\right)+\int_{0}^{t} B(t-s) \mathcal{D}\left(s, x_{s}\right) d s+g\left(t, x_{t}\right), t \in(0, a), \\
x_{0} & =\varphi \in \Omega \subset \mathcal{B},
\end{aligned}
$$

onde $A: D(A) \subset X \rightarrow X, B(t): D(B(t)) \subset X \rightarrow X t \geq 0$ são operadores fechados em $X$ com $D(B(t)) \supset D(A)$ para todo $t \geq 0 ; \mathcal{B}$ é um espaço de fase definido de maneira axiomática; $\Omega \subset \mathcal{B}$ é aberto; $\mathcal{D}(t, \varphi)=\varphi(0)+f(t, \varphi)$ e $f, g:[0, a] \times \Omega \rightarrow X$ são funções apropriadas.
\end{abstract}

\subsection{Existência de soluções fracas}

Neste capítulo assumiremos que $(R(t))_{t \geq 0}$ é uma família resolvente para 1.1 -1.2 . Assumiremos também que $M>0$ é tal que $\|R(t)\| \leq M$ para todo $t \in[0, a]$. No que segue $\mathcal{B}$ é um espaço de fase abstrato que verifica os axiomas (A), (A1) e (B) do Capítulo 1 e $H, K(\cdot), M(\cdot)$ são como no axioma (A). Adicionalmente, para uma função limitada $\xi$ : 
$[0, b] \rightarrow[0, \infty)$ e $t \in[0, b]$, usaremos a notação $\xi_{t}$ para $\xi_{t}=\sup \{\xi(s): s \in[0, t]\}$. Para um espaço de Banach $\left(Z,\|\cdot\|_{Z}\right)$ e uma função contínua $\eta:[0, b] \rightarrow Z$ usaremos a notação $\|\eta(\theta)\|_{t}=\sup \{\|\eta(\theta)\|: \theta \in[0, t]\}$. Por $B_{r}(x, Z)$ denotaremos a bola fechada de centro em $x$ e raio $r$ no espaço $Z$ e por $B_{r}(\psi, \mathcal{B})$ denotaremos a bola de centro em $\psi$ e raio $r$ no espaço de fase $\mathcal{B}$.

Começamos introduzindo o conceito de solução fraca para o sistema (2.1)-(2.2).

Definição 2.1.1 Uma função $x:(-\infty, b] \rightarrow X$ é chamada de solução fraca de (2.1)- (2.2) sobre $[0, b], 0<b \leq a$, se $x \in C([0, b]: X) ; x_{0}=\varphi$ e

$$
x(t)=R(t)(\varphi(0)+f(0, \varphi))-f\left(t, x_{t}\right)+\int_{0}^{t} R(t-s) g\left(s, x_{s}\right) d s, \quad t \in[0, b] .
$$

Observação 2.1.1 No que segue, $y:(-\infty, a] \rightarrow X$ é a função definida por

$$
y(t)=\left\{\begin{array}{rcc}
R(t) \varphi(0) & \text { se } & 0 \leq t \leq a \\
\varphi(t) & \text { se } & -\infty<t<0 .
\end{array}\right.
$$

Agora estamos em condições de estabelecer nosso primeiro resultado de existência de soluções fracas, para isto usaremos o clássico Princípio da Contração.

Teorema 2.1.1 Suponha que as funções $f, g:[0, a] \times \Omega \rightarrow X$ são contínuas e que existem constantes positivas $L_{f}, L_{g}$ tais que

$$
\begin{aligned}
\left\|f\left(t, \psi_{1}\right)-f\left(t, \psi_{2}\right)\right\| & \leq L_{f}\left\|\psi_{1}-\psi_{2}\right\|_{\mathcal{B}} \\
\left\|g\left(t, \psi_{1}\right)-g\left(t, \psi_{2}\right)\right\| & \leq L_{g}\left\|\psi_{1}-\psi_{2}\right\|_{\mathcal{B}}
\end{aligned}
$$

para todo $t \in[0, a]$ e todo $\psi_{1}, \psi_{2} \in \Omega$.

Se $K(0) L_{f}<1$, então existe uma única solução fraca do problema (2.1)-(2.2) sobre $[0, b]$ para algum $0<b \leq a$.

Demonstração: Usando que $f$ e $g$ são contínuas, fixamos constantes $b_{\varphi}, r_{1}, C_{f}$ e $C_{g}$ tais que $B_{r_{1}}(\varphi, \mathcal{B}) \subseteq \Omega$ e $\|f(t, \psi)\| \leq C_{f},\|g(t, \psi)\| \leq C_{g}$, para todo $0 \leq t \leq b_{\varphi}$ e todo $\psi \in B_{r_{1}}(\varphi, \mathcal{B})$.

Seja $y(\cdot)$ a função introduzida em (2.4). Pelo axioma (A1), a função $t \rightarrow y_{t}$ é contínua em $\left[0, b_{\varphi}\right]$, assim podemos fixar $\rho>0$ e $0<b_{1}<b_{\varphi}$ tais que

$$
\begin{aligned}
\mu & =L_{f} K_{b_{1}}<1 \\
K_{b_{1}} \rho & +\sup _{t \in\left[0, b_{1}\right]}\left\|y_{t}-\varphi\right\|_{\mathcal{B}}<r_{1} .
\end{aligned}
$$


Fixemos agora $0<b \leq b_{1}$ tal que as seguintes desigualdades sejam verifidas.

$$
\begin{array}{r}
\|(R(\theta)-I) f(0, \varphi)\|_{b} \leq \frac{(1-\mu) \rho}{3}, \\
\left\|f\left(\theta, y_{\theta}\right)-f(0, \varphi)\right\|_{b} \leq \frac{(1-\mu) \rho}{3}, \\
M b C_{g} \leq \frac{(1-\mu) \rho}{3}, \\
K_{b}\left(L_{f}+M L_{g} b\right)<1 .
\end{array}
$$

Sobre o espaço

$$
S(b)=\left\{u:(-\infty, b] \rightarrow X:\left.u\right|_{[0, b]} \in C([0, b], X), u_{0}=0,\|u(\theta)\|_{b} \leq \rho\right\},
$$

munido com a norma da convergência uniforme, definimos o operador $\Gamma: S(b) \rightarrow C([0, b]: X)$ por

$$
\Gamma x(t)=R(t) f(0, \varphi)-f\left(t, x_{t}+y_{t}\right)+\int_{0}^{t} R(t-s) g\left(s, x_{s}+y_{s}\right) d s .
$$

No que segue, mostraremos que $\Gamma$ é uma contração de $S(b)$ em $S(b)$. É claro que para $x \in S(b), \Gamma x$ é contínua em $[0, b]$. Por outro lado, se $x \in S(b)$, de 2.6 temos que

$$
\left\|x_{t}+y_{t}-\varphi\right\|_{\mathcal{B}} \leq\left\|x_{t}\right\|_{\mathcal{B}}+\left\|y_{t}-\varphi\right\|_{\mathcal{B}} \leq K_{b_{1}} \rho+\sup _{t \in\left[0, b_{1}\right]}\left\|y_{t}-\varphi\right\|_{\mathcal{B}}<r_{1},
$$

o que mostra que $x_{t}+y_{t} \in B_{r_{1}}(\varphi, \mathcal{B})$ para todo $t \in[0, b]$. Assim temos que $\left\|f\left(t, x_{t}+y_{t}\right)\right\|<C_{f}$ e $\left\|g\left(t, x_{t}+y_{t}\right)\right\|<C_{g}$ para todo $t \in[0, b]$ e todo $x \in S(b)$. Logo para $x \in S(b)$ e $t \in[0, a]$ vemos que

$$
\begin{aligned}
\|\Gamma x(t)\| \leq & \|R(t) f(0, \varphi)-f(0, \varphi)\|+\left\|f\left(t, x_{t}+y_{t}\right)-f\left(t, y_{t}\right)\right\| \\
& +\left\|f\left(t, y_{t}\right)-f(0, \varphi)\right\|+\left\|\int_{0}^{t} R(t-s) g\left(s, x_{s}+y_{s}\right) d s\right\| \\
\leq & \frac{(1-\mu) \rho}{3}+L_{f}\left\|x_{t}\right\|_{\mathcal{B}}+\frac{(1-\mu) \rho}{3}+M b C_{g} \\
\leq & \frac{(1-\mu) \rho}{3}+L_{f} K_{b}\|x(\theta)\|_{b}+\frac{(1-\mu) \rho}{3}+\frac{(1-\mu) \rho}{3} \\
= & (1-\mu) \rho+\mu \rho=\rho,
\end{aligned}
$$

o que prova que $\Gamma x \in S(b)$ e então que $\Gamma(S(b)) \subset S(b)$. Mais ainda se $u, v \in S(b)$, tem-se que

$$
\begin{aligned}
\|\Gamma u(t)-\Gamma v(t)\| \leq & \left\|f\left(t, u_{t}+y_{t}\right)-f\left(t, v_{t}+y_{t}\right)\right\| \\
& +\int_{0}^{t} M\left\|g\left(s, u_{s}+y_{s}\right)-g\left(s, v_{s}+y_{s}\right)\right\| d s \\
\leq & L_{f}\left\|u_{t}-v_{t}\right\|_{\mathcal{B}}+L_{g} M \int_{0}^{t}\left\|u_{s}-v_{s}\right\|_{\mathcal{B}} d s \\
\leq & K_{b}\left(L_{f}+L_{g} M b\right)\|u(\theta)-v(\theta)\|_{b},
\end{aligned}
$$


o que prova que $\|\Gamma u(\theta)-\Gamma v(\theta)\|_{b}<K_{b}\left(L_{f}+L_{g} M b\right)\|u(\theta)-v(\theta)\|_{b}$. Portanto, $\Gamma$ é uma contração de $S(b)$ em $S(b)$ por 2.10). Do princípio da Contração, existe um único ponto fixo $x \in X$ de $\Gamma$. É claro que $u=x+y$ é a única solução fraca de 2.1 - 2.2. Assim a prova do Teorema está completa.

Para mostrar nossos próximos resultados de existência de soluções fracas, é necessário considerar o seguinte lema que estabelece condições nas quais um certo operador do tipo convolução é completamente contínuo.

Lema 2.1.1 Sejam $\left(Z_{i},\|\cdot\|_{Z_{i}}\right), i=1,2,3$, espaços de Banach, $\beta>0 ;(C(t))_{t \in[0, \beta]}$ uma familia de operadores em $\mathcal{L}\left(Z_{2}, Z_{3}\right)$ e $f:[0, \beta] \times Z_{1} \rightarrow Z_{2}$. Assuma que as seguintes condições são verificadas

(a) Para cada $x \in Z_{2}$, a função $t \rightarrow C(t) x$ é contínua sobre $(0, \beta]$ e existe $J \in L^{1}\left([0, \beta]: \mathbb{R}^{+}\right)$ tal que $\|C(t)\|_{\mathcal{L}\left(Z_{2}, Z_{3}\right)} \leq J(t)$ para todo $t \in[0, \beta]$

(b) A função $f(\cdot, x)$ é fortemente mensurável sobre $[0, \beta]$ para cada $x \in Z_{1}$ e a função $f(s, \cdot): Z_{1} \rightarrow Z_{2}$ é contínua para todo $s \in[0, \beta] ;$

(c) Existe uma função contínua $m:[0, \infty) \rightarrow[0, \infty)$ e uma função contínua e não decrescente $\Omega:[0, \infty) \rightarrow(0, \infty)$ tal que $\|f(t, x)\|_{Z_{2}} \leq m(t) \Omega\left(\|x\|_{Z_{1}}\right)$ para todo $(t, x) \in[0, \beta] \times Z_{1}$.

Seja $\Gamma: C\left([0, \beta]: Z_{1}\right) \rightarrow C\left([0, \beta], Z_{3}\right)$ o operador definido por

$$
\Gamma x(t)=\int_{0}^{t} C(t-s) f(s, x(s)) d s .
$$

Então $\Gamma$ é contínua. Mais ainda, se alguma das seguintes condições é verificada,

(i) A função $f(\cdot)$ é completamente contínua;

(ii) A função $t \rightarrow C(t)$ é contínua em $\mathcal{L}\left(Z_{2}, Z_{3}\right)$ sobre $(0, \beta]$ e para cada $t \in(0, \beta]$ e $r>0$ o conjunto $\left\{C(t) f(s, x(s)): s \in[0, \beta], x \in B_{r}\left(0, Z_{1}\right)\right\}$ é relativamente compacto em $Z_{3}$,

então $\Gamma$ é um operador completamente contínuo.

Demonstração: Usando (a), (b) e (c) é fácil ver que $\Gamma x \in C\left([0, \beta]: Z_{3}\right)$ se $x \in C([0, \beta]$ : $\left.Z_{1}\right)$. Para mostrar que $\Gamma$ é contínua, fixemos uma seqüência $\left(u_{n}\right)_{n \in \mathbb{N}}$ em $C\left([0, \beta]: Z_{1}\right)$ e 
$u \in C\left([0, \beta]: Z_{1}\right)$ tal que $u_{n} \rightarrow u$ quando $n \rightarrow \infty$. É obvio que $f\left(s, u_{n}(s)\right) \rightarrow f(s, u(s))$ q.t.p. sobre $[0, \beta]$. Isto junto a estimativa

$$
\left\|C(t-s) f\left(s, u_{n}(s)\right)\right\|_{Z_{3}} \leq J(t-s) m(s) W\left(\left\|u_{n}(s)\right\|_{Z_{1}}\right) \leq J(t-s) m(s) W(L),
$$

onde $L \geq \sup _{s \in[0, \beta], n \in \mathbb{N}}\left\|u_{n}(s)\right\|_{Z_{1}}$, e o Teorema da convergência dominada, nos permite concluir que $\Gamma$ é contínua.

Para mostrar que $\Gamma$ é um operador compacto usaremos o clássico Teorema de AscoliArzela. Para isto, fixamos $B_{r}=B_{r}\left(0, C\left([0, \beta]: Z_{1}\right)\right), r>0$. No que segue estudamos separadamente a equicontinuidade de $\Gamma B_{r}$ e a pre-compacidade do conjunto $\Gamma B_{r}(t)=\{\Gamma x(t)$ : $\left.x \in B_{r}\right\}$.

Suponha inicialmente que (i) é verificada. Mostraremos que $\Gamma\left(B_{r}\right)(t)$ é relativamente compacto para todo $t \in[0, \beta]$. Sejam $0<\epsilon<t \leq \beta$. Como a função $s \rightarrow C(s)$ é fortemente contínua sobre $[0, \beta]$, segue que o cojunto $W=\left\{C(s) y: \quad s \in[\epsilon, \beta], \quad y \in f\left([0, \beta] \times B_{r}\right)\right\}$ é relativamente compacto em $Z_{3}$. Usando agora o Teorema 1.3.3, vemos que para $x \in B_{r}$

$$
\begin{aligned}
\Gamma x(t) & =\int_{0}^{\epsilon} C(s) f(t-s, x(t-s)) d s+\int_{\epsilon}^{t} C(s) f(t-s, x(t-s)) d s \\
& \in C_{\epsilon}+(t-\epsilon) \overline{c o(W)},
\end{aligned}
$$

onde $\operatorname{diam}\left(C_{\epsilon}\right) \leq W(r) \int_{0}^{\epsilon} J(s) m(t-s) d s$. Isto prova que $\left\{\Gamma x(t): x \in B_{r}\right\}$ é totalmente limitado em $Z_{3}$ e portanto relativamente compacto. Como $\Gamma B_{r}(0)=\{0\}$, deduzimos que a propriedade é válida para todo $t \in[0, \beta]$.

Mostraremos agora que $\Gamma B_{r}$ é equicontínuo sobre $[0, \beta]$. Seja $t \in(0, \beta]$ e fixemos $0<$ $\epsilon<t$. Usando que $U=\left\{f(s, y):(s, y) \in[0, \beta] \times B_{r}\right\}$ é relativamente compacto em $Z_{2}$, e que $s \rightarrow C(s) x$ é contínua sobre $[\epsilon, \beta]$ para todo $x \in Z_{2}$, segue que o conjunto de funções $\{s \rightarrow$ $C(s) x: x \in U\}$ é equicontínuo sobre $[\epsilon, \beta]$. Assim, existe $\delta>0$ tal que $\left\|C(s) x-C\left(s^{\prime}\right) x\right\|_{z_{3}}<$ $\epsilon$, para todo $s, s^{\prime} \in[\epsilon, \beta]$ e todo $x \in U$ tal que $\left|s-s^{\prime}\right|<\delta$. Nestas condições, para $x \in B_{r}$ e $0<h<\delta$ obtemos que

$$
\begin{aligned}
& \|\Gamma x(t+h)-\Gamma x(t)\|_{Z_{3}} \leq \int_{0}^{t-\epsilon}\|C(t+h-s)-C(t-s) f(s, x(s))\|_{Z_{2}} d s \\
& +\int_{t-\epsilon}^{t}\|C(t+h-s)-C(t-s)\|_{\mathcal{L}\left(Z_{2}, Z_{3}\right)}\|f(s, x(s))\|_{Z_{2}} d s \\
& +\int_{t}^{t+h}\|C(t+h-s)\|_{\mathcal{L}\left(Z_{2}, Z_{3}\right)}\|f(s, x(s))\|_{Z_{2}} d s \\
& \leq W(r)\left(\epsilon \int_{0}^{t-\epsilon} m(s) d s+\int_{t-\epsilon}^{t}(J(t+h-s)+J(t-s)) m(s) d s\right) \\
& +W(r) \int_{t}^{t+h} J(t+h-s) m(s) d s,
\end{aligned}
$$


o que nos permite concluir que $\Gamma B_{r}$ é equicontínuo a direita em $t \in(0, \beta]$. Procedendo de forma similar podemos mostrar a equicontinuidade em $t=0$ e a equicontinuidade a esquerda em $t \in(0, \beta]$. Portanto, $\Gamma B_{r}$ é equicontínuo sobre $[0, \beta]$. Segue dos passos anteriores que $\Gamma$ é completamente contínuo quando (i) é válido.

Suponha válida a condição (ii). Mostraremos para começar que o conjunto $\Gamma B_{r}(t)$ é relativamente compacto. Sejam $t \in(0, \beta]$ e $0<\epsilon<t$. Como $t \rightarrow C(t)$ é contínua sobre $[\epsilon, \beta]$, existem pontos $\epsilon=t_{1}<t_{2}<\ldots<t_{n+1}=t$, tais que $\left\|C(s)-C\left(s^{\prime}\right)\right\|_{\mathcal{L}\left(Z_{2}, Z_{3}\right)} \leq \epsilon$ se $s, s^{\prime} \in\left[t_{i}, t_{i+1}\right]$ para algum $i=1,2, \ldots, n$. Nessas condições, do Teorema do valor medio para a integral de Bochner, veja Teorema 1.3 .3 , para $x \in B_{r}$ vemos que

$$
\begin{aligned}
\Gamma x(t)= & \int_{\epsilon}^{t} C(s) f(t-s, x(t-s)) d s+\int_{0}^{\epsilon} C(s) f(t-s, x(t-s)) d s \\
= & \sum_{i=1}^{n} \int_{t_{i}}^{t_{i+1}}\left(C(s)-C\left(t_{i}\right)\right) f(t-s, x(t-s)) d s+\sum_{i=1}^{n} \int_{t_{i}}^{t_{i+1}} C\left(t_{i}\right) f(t-s, x(t-s)) d s \\
& +\int_{0}^{\epsilon} C(s) f(t-s, x(t-s)) d s \\
\in & \sum_{i=1}^{n} C_{\epsilon}^{i}+\sum_{i=1}^{n}\left(t_{i+1}-t_{i}\right) \overline{c o\left(\left\{C\left(t_{i}\right) f(\xi, y): \xi \in[0, \beta], y \in B_{r}\left(0, Z_{1}\right)\right\}\right)}+B_{\epsilon}
\end{aligned}
$$

onde $\operatorname{diam}\left(C_{\epsilon}^{i}\right) \leq \epsilon W(r) \int_{0}^{\beta} m(t-s) d s$ e $\operatorname{diam}\left(B_{\epsilon}\right) \leq W(r) \int_{0}^{\epsilon} J(s) m(t-s) d s$. Isto nos permite concluir que $\Gamma\left(B_{r}\right)(t)$ é totalmente limitado em $Z_{3}$ e como conseqüência que $\Gamma B_{r}(t)$ é relativamente compacto em $Z_{3}$ para todo $t \in[0, \beta]$, pois $\Gamma B_{r}(0)=\{0\}$.

Vejamos agora que $\Gamma B_{r}$ é equicontinuo sobre $[0, \beta]$. Seja $t \in(0, \beta)$. Como a função $s \rightarrow C(s)$ é contínua sobre $[\epsilon, \beta]$, existe $\delta>0$ tal que $\left\|C(s)-C\left(s^{\prime}\right)\right\|_{\mathcal{L}\left(Z_{2}, Z_{3}\right)}<\epsilon$, para todo $s, s^{\prime} \in[\epsilon, \beta]$ quando $\left|s-s^{\prime}\right|<\delta$. Usando isto, para $x \in B_{r}$ e $0<h<\delta$ com $t+h \in(0, \beta)$ vemos que

$$
\begin{aligned}
\|\Gamma x(t+h)-\Gamma x(t)\|_{Z_{3} \leq} \leq & \int_{0}^{t-\epsilon}\|C(t+h-s)-C(t-s)\|_{\mathcal{L}\left(Z_{2}, Z_{3}\right)}\|f(s, x(s))\|_{Z_{2}} d s \\
& +\int_{t-\epsilon}^{t}\|C(t+h-s)-C(t-s)\|_{\mathcal{L}\left(Z_{2}, Z_{3}\right)}\|f(s, x(s))\|_{Z_{2}} d s \\
& +\int_{t}^{t+h}\|C(t+h-s)\|_{\mathcal{L}\left(Z_{2}, Z_{3}\right)}\|f(s, x(s))\|_{Z_{2}} d s \\
\leq & W(r)\left(\epsilon \int_{0}^{t-\epsilon} m(s) d s+\int_{t-\epsilon}^{t}(J(t+h-s)+J(t-s)) m(s) d s\right) \\
& +W(r) \int_{t}^{t+h} J(t+h-s) m(s) d s,
\end{aligned}
$$

o que permite concluir que $\Gamma B_{r}$ é equicontínuo a direita em $t \in(0, \beta)$. Usando um argumento similar podemos mostrar a equicontinuidade em $t=0$ e a equicontinuidade a esquerda em 
$t \in(0, \beta]$. Portanto, $\Gamma B_{r}$ é equicontínuo. Segue do anterior que se (ii) é válido, então $\Gamma$ é completamente contínuo. Agora a prova está completa.

Para mostrar nossos próximos Teoremas introduzimos a seguinte hipótese sobre a função $g(\cdot)$.

$\left(\mathbf{H}_{\mathbf{g}}\right)$ A função $g:[0, a] \times \mathcal{B} \rightarrow X$ satisfaz as seguintes condições.

(i) A função $g(t, \cdot): \mathcal{B} \rightarrow X$ é contínua para cada $t \in[0, a]$;

(ii) Para cada $x \in X$, a função $g(\cdot, x):[0, a] \rightarrow X$ é fortemente mensurável;

(iii) Existe uma função contínua $m_{g}:[0, a] \rightarrow[0, \infty)$ e uma função contínua não decrescente $\Omega_{g}:[0, \infty) \rightarrow(0, \infty)$ tal que

$$
\|g(t, \psi)\| \leq m_{g}(t) \Omega_{g}\left(\|\psi\|_{\mathcal{B}}\right), \quad(t, \psi) \in[0, a] \times \mathcal{B} .
$$

Para a prova do nosso próximo resultado usaremos o Teorema 1.3.1.

Teorema 2.1.2 Suponha que a hipótese $\left(\mathbf{H}_{\mathbf{g}}\right)$ é válida e que as seguintes condições são verificadas

(a) A função $f:[0, a] \times \Omega \rightarrow X$ é contínua e existe $L_{f}>0$ tal que

$$
\left\|f\left(t, \psi_{1}\right)-f\left(t, \psi_{2}\right)\right\| \leq L_{f}\left\|\psi_{1}-\psi_{2}\right\|_{\mathcal{B}}
$$

para todo $t \in[0, a]$ e todo $\psi_{1}, \psi_{2} \in \Omega$;

(b) Existe uma constante $r_{\varphi}>0$, tal que para cada $t \in[0, a]$ existe um compacto $W_{t} \subseteq X$ tal que $R(t) g(s, \psi) \in W_{t}$ para todo $\psi \in B_{r(\varphi)}(\varphi, \mathcal{B})$ e todo $s \in[0, a]$.

Se $K(0) L_{f}<1$, então existe uma solução fraca do problema 2.1-2.2 sobre $[0, b]$ para algum $0<b \leq a$.

Demonstração: Sejam $S(b), \rho, r$ e $\Gamma(\cdot)$ como na prova do Teorema 2.1.1. Sem perda de generalidade assumiremos que $r<r_{\varphi}$. Considere a decomposição $\Gamma=\Gamma_{1}+\Gamma_{2}$, onde

$$
\begin{aligned}
& \Gamma_{1} x(t)=R(t) f(0, \varphi)-f\left(t, x_{t}+y_{t}\right), \\
& \Gamma_{2} x(t)=\int_{0}^{t} R(t-s) g\left(s, x_{s}+y_{s}\right) d s .
\end{aligned}
$$

Da prova do Teorema 2.1.1 sabemos que $\Gamma_{1}$ é uma contração de $S(b)$ em $S(b)$. Pelo Lema 2.1.1, deduzimos que $\Gamma_{2}$ é completamente contínuo de $S(b)$ em $S(b)$. Assim, $\Gamma$ é um 
operador condensante o que pelo Teorema 1.3.1 permite concluir que $\Gamma$ possui um ponto fixo $x \in S(b)$. É claro que $u=x+y$ é solução fraca de 2.1)-2.2. A prova está completa.

Para a demostração do nosso próximo Teorema introduzimos a seguinte hipótese sobre a função $f(\cdot)$.

$\left(\mathbf{H}_{\mathbf{f}}\right)$ A função $f:[0, a] \times \mathcal{B} \rightarrow X$ é completamente contínua e existem constantes $c_{1}, c_{2}>0$ tais que

$$
\|f(t, \psi)\| \leq c_{1}\|\psi\|_{\mathcal{B}}+c_{2}, \quad(t, \psi) \in[0, a] \times \mathcal{B}
$$

Seja $y(\cdot)$ a função definida em 2.4 e $S(a)=\left\{x:(-\infty, a] \rightarrow X: x_{0}=0,\left.x\right|_{[0, a]} \in\right.$ $C([0, a]: X)\}$ munido com a norma da convergência uniforme. Para todo $Q \subset S(a)$ limitado, o conjunto de funções $\left\{t \rightarrow f\left(t, x_{t}+y_{t}\right): x \in Q\right\}$ é equicontínuo sobre $[0, a]$.

Teorema 2.1.3 Suponha que as hipótese $\left(\mathbf{H}_{\mathbf{f}}\right),\left(\mathbf{H}_{\mathbf{g}}\right)$ e a condição $(\mathbf{b})$ do Teorema 2.1.2 são verificadas. Assuma também que

$$
\frac{M K_{a}}{\mu} \int_{0}^{t} m_{g}(s) d s<\int_{\frac{C}{\mu}}^{\infty} \frac{d s}{\Omega_{g}(s)}
$$

onde $C=\left(\left(M_{a}+K_{a} M H\right)+M c_{1} K_{a}\right)\|\varphi\|_{\mathcal{B}}+(M+1) c_{2} K_{a}$ e $\mu=1-K_{a} c_{1}>0$. Então existe uma solução fraca do problema (2.1)- (2.2) sobre $[0, a]$.

Demonstração: Sobre o espaço $S(a)$ definimos o operador $\Gamma: S(a) \rightarrow S(a)$ por

$$
\Gamma x(t)=\left\{\begin{array}{cl}
0, & \text { se } \quad t<0, \\
R(t) f(0, \varphi)-f\left(t, x_{t}+y_{t}\right)+\int_{0}^{t} R(t-s) g\left(s, x_{s}+y_{s}\right) d s, & \text { se } \quad 0 \leq t \leq a .
\end{array}\right.
$$

Para mostrar nosso resultado, usaremos o Teorema 1.3.2, Inicialmente, obtemos estimativas a priori para as soluções da equação integral $x=\lambda \Gamma x, \lambda \in(0,1)$. Seja $x^{\lambda}$ uma solução de $x=\lambda \Gamma x$. Usando a notação $v^{\lambda}(s)=\left(M_{a}+K_{a} M H\right)\|\varphi\|_{\mathcal{B}}+K_{a}\left\|x^{\lambda}(\theta)\right\|_{s}$, dos axiomas do espaço de fase segue que

$$
\begin{aligned}
\left\|x^{\lambda}(t)\right\| & \leq\|R(t) f(0, \varphi)\|+\left\|f\left(t, x_{t}^{\lambda}+y_{t}^{\lambda}\right)\right\|+\left\|\int_{0}^{t} R(t-s) g\left(s, x_{s}^{\lambda}+y_{s}^{\lambda}\right) d s\right\| \\
& \leq M\left(c_{1}\|\varphi\|_{\mathcal{B}}+c_{2}\right)+\left(c_{1}\left\|x_{t}^{\lambda}+y_{t}^{\lambda}\right\|_{\mathcal{B}}+c_{2}\right)+\int_{0}^{t} M m_{g}(s) \Omega_{g}\left(\left\|x_{s}^{\lambda}+y_{s}^{\lambda}\right\|_{\mathcal{B}}\right) d s \\
& \leq\left(M c_{1}\|\varphi\|_{\mathcal{B}}+(M+1) c_{2}\right)+c_{1}\left\|x_{t}^{\lambda}+y_{t}^{\lambda}\right\|_{\mathcal{B}}+\int_{0}^{t} M m_{g}(s) \Omega_{g}\left(\left\|x_{s}^{\lambda}+y_{s}^{\lambda}\right\|_{\mathcal{B}}\right) d s \\
& \leq\left(M c_{1}\|\varphi\|_{\mathcal{B}}+(M+1) c_{2}\right)+c_{1} v^{\lambda}(t)+\int_{0}^{t} M m_{g}(s) \Omega_{g}\left(v^{\lambda}(s)\right) d s .
\end{aligned}
$$


Da definição de $v^{\lambda}$ temos agora que

$$
\begin{aligned}
v^{\lambda}(t) \leq & \left(M_{a}+K_{a} M H\right)\|\varphi\|_{\mathcal{B}}+K_{a}\left\|x^{\lambda}(\theta)\right\|_{s} \\
\leq & \left(\left(M_{a}+K_{a} M H\right)+M c_{1} K_{a}\right)\|\varphi\|_{\mathcal{B}}+(M+1) c_{2} K_{a}+K_{a} c_{1} v^{\lambda}(t) \\
& +M K_{a} \int_{0}^{t} m_{g}(s) \Omega_{g}\left(v^{\lambda}(s)\right) d s,
\end{aligned}
$$

e portanto

$$
v^{\lambda}(t) \leq \frac{C}{\mu}+\frac{M K_{a}}{\mu} \int_{0}^{t} m_{g}(s) \Omega_{g}\left(v^{\lambda}(s)\right) d s .
$$

Seja $\beta_{\lambda}(t)$ o lado direito da desigualdade anterior. Derivando $\beta_{\lambda}$ temos que

$$
\beta_{\lambda}^{\prime}(t) \leq \frac{M K_{a}}{\mu} m_{g}(t) \Omega_{g}\left(\beta_{\lambda}(t)\right)
$$

e assim que

$$
\frac{\beta_{\lambda}^{\prime}(t)}{\Omega_{g}\left(\beta_{\lambda}(t)\right)} \leq \frac{M K_{a}}{\mu} m_{g}(t)
$$

Portanto,

$$
\int_{\beta_{\lambda}(0)}^{\beta_{\lambda}(t)} \frac{d s}{\Omega_{g}(s)} \leq \frac{M K_{a}}{\mu} \int_{0}^{t} m_{g}(s) d s<\int_{\frac{C}{\mu}}^{\infty} \frac{d s}{\Omega_{g}(s)}, \quad t \in[0, a] .
$$

Da última desigualdade e de 2.11, podemos concluir que o conjunto $\left\{\beta_{\lambda}(t): t \in[0, a] ; \lambda \in\right.$ $(0,1)\}$ é limitado o que mostra que o conjunto $\left\{x^{\lambda}(\cdot): \lambda \in(0,1)\right\}$ é limitado em $S(a)$.

Para mostrar que $\Gamma$ é um operador completamente contínuo, introduzimos a decomposição $\Gamma=\Gamma_{1}+\Gamma_{2}$, onde $\left(\Gamma_{i} x\right)_{0}=0, i=1,2$, e

$$
\begin{array}{ll}
\Gamma_{1} x(t)=R(t) f(0, \varphi)-f\left(t, x_{t}+y_{t}\right), & t \in[0, a], \\
\Gamma_{2} x(t)=\int_{0}^{t} R(t-s) g\left(s, x_{s}+y_{s}\right) d s, & t \in[0, a] .
\end{array}
$$

Devido as propriedades do operador resolvente e as hipóteses $\left(\mathbf{H}_{\mathbf{f}}\right)$ e $\left(\mathbf{H}_{\mathbf{g}}\right)$ temos que $\Gamma_{1}$ e $\Gamma_{2}$ são bem definidas e assumem valores em $S(a)$. Mais adiante, das propriedades de $f$ e do Lema 2.1.1 segue que os operadores $\Gamma_{i}, i=1,2$, são compactos. Assim, para completar a prova, é suficiente mostrar que cada $\Gamma_{i}, i=1,2$, é contínuo. Sejam $\left(u^{n}\right)_{n \in \mathbb{N}}$ uma seqüência em $S(a)$ e $u \in S(a)$ tais que $u^{n} \rightarrow u$ em $S(a)$. Dos axiomas do espaço de fase, deduzimos que o conjunto $U=\left\{u_{s}^{n}, u_{s}: s \in[0, a], n \in \mathbb{N}\right\}$ é relativamente compacto em $\mathcal{B}$. Como $f$ é uniformemente contínua sobre $U$, segue que $f\left(s, u_{s}^{n}\right) \rightarrow f\left(s, u_{s}\right)$ uniformemente sobre $[0, a]$ quando $n \rightarrow \infty$, o que mostra que $\Gamma_{1}$ é contínua. 
Para provar a continuidade de $\Gamma_{2}$, fixamos $r>0$ tal que $\left\|u_{s}^{n}\right\|_{\mathcal{B}} \leq r$ para todo $n \in \mathbb{N}$ e todo $s \in[0, a]$. Como $\left\|g\left(s, x_{s}\right)\right\| \leq m_{g}(s) \Omega_{g}\left(r+\sup _{s \in[0, a]}\left\|y_{s}\right\|_{\mathcal{B}}\right)$, podemos deduzir a continuidade de $\Gamma_{2}$ a partir do Teorema da convergência dominada de Lebesgue.

Como conseqüência do anterior, obtemos que $\Gamma$ é um operador completamente contínuo sobre $S(a)$ e do Teorema 1.3.2, que $\Gamma$ possui um ponto fixo $x \in S(a)$. Agora a prova do resultado está completa pois $u=x+y$ é uma solução fraca de (2.1)-2.2.

\subsection{Resultados de regularidade}

Nesta seção estudaremos a regularidade das soluções fracas de (2.1)-(2.2) obtidas na seção anteior. Para isto assumiremos que $(R(t))_{t \geq 0}$ é um operador resolvente exponencialmente limitado para $1.1-1.2$. Por simplicidade, no que segue assumiremos que $\Omega=\mathcal{B}$.

\subsubsection{Existência de solução semi-clássica}

Para começar, introduzimos o seguinte conceito se solução.

Definição 2.2.1 Uma função $u:(-\infty, b) \rightarrow X$ é uma solução semi-clássica de 2.1)-2.2) se $u_{0}=\varphi ;$ as funções $u(\cdot)$ e $t \rightarrow f\left(t, u_{t}\right)$ são funções em $W^{1,1}([0, b]: X) ;$ a função $t \rightarrow$ $u(t)+f\left(t, u_{t}\right) \in C([0, b): D(A)) \cap C^{1}((0, b): X)$ e 2.1) é satisfeita em $[0, b)$.

Para provar nosso primeiro resultado de regularidade precisamos de algumas preliminares. No próximo Lema, $W(\cdot)$ e $S(\cdot)$ são as famílias de operadores definidas em (1.6) e 1.7 respectivamente. Como antes, $y:(-\infty, a] \rightarrow X$ é a função introduzida em (2.4).

Lema 2.2.1 Se as funções $R(\cdot) \varphi(0)$ e $S(\cdot) \varphi$ são Lipschitz em $[0, a]$, então a função $s \rightarrow y_{s}$ também é Lipshitz em $[0, a]$.

Demonstração: É obvio que $y_{t}=W(t) \varphi$ para $t \geq 0$. Se $s \in[0, a]$ e $h>0$ são tais que $s+h \in[0, a]$, então

$$
\begin{aligned}
\left\|y_{s+h}-y_{s}\right\|_{\mathcal{B}} & \leq M_{a}\left\|y_{h}-\varphi\right\|_{\mathcal{B}}+\|y(\theta+h)-y(\theta)\|_{a} \\
& \leq M_{a}\|W(h) \varphi-S(h) \varphi\|_{\mathcal{B}}+M_{a}\|S(h) \varphi-\varphi\|_{\mathcal{B}}+C_{1} h \\
& \leq M_{a} K_{a}\|R(\theta) \varphi(0)-\varphi(0)\|_{h}+C_{2} h \\
& \leq C_{3} h,
\end{aligned}
$$


onde as constantes $C_{i}, i=1,2,3$ são independentes de $t$ e $h$. Isto prova que $s \rightarrow y_{s}$ é Lipschitz sobre $[0, a]$.

Lema 2.2.2 Assuma que as condições do Lema 2.2.1 são verificadas. Suponha também que a função $t \rightarrow R(t) f(0, \varphi)$ é Lipschitz em $[0, a]$ e que existem constantes $L_{f}, L_{g}>0$ tais que

$$
\begin{aligned}
\left\|f\left(t, \psi_{1}\right)-f\left(s, \psi_{2}\right)\right\| & \leq L_{f}\left(|t-s|+\left\|\psi_{1}-\psi_{2}\right\|_{\mathcal{B}}\right), \\
\left\|g\left(t, \psi_{1}\right)-g\left(s, \psi_{2}\right)\right\| & \leq L_{g}\left(|t-s|+\left\|\psi_{1}-\psi_{2}\right\|_{\mathcal{B}}\right),
\end{aligned}
$$

para todo $0 \leq s, t \leq$ a e todo $\psi_{1}, \psi_{2} \in \mathcal{B}$. Se $x(\cdot)$ é uma solução fraca de (2.1)-(2.2) sobre $[0, b], 0<b \leq a$, e $K_{b} L_{f}<1$, então as funções $x(\cdot)$ e $s \rightarrow x_{s}$ são Lipschitz sobre $[0, b]$.

Demonstração: Considere a desomposição $x(t)=y(t)+z(t)$ onde $y$ é a função introduzida em (2.4). Para começar vejamos que $z(\cdot)$ é Lipschitz sobre $[0, b]$. Usando que as funções $y(\cdot)$ e $t \rightarrow y_{t}$ são Lipschitz, veja Lema 2.2.1, para $t \in[0, b]$ e $h>0$ tais que $t+h \in[0, b]$ temos que

$$
\begin{aligned}
\|z(t+h)-z(t)\| \leq & \|R(t+h) f(0, \varphi)-R(t) f(0, \varphi)\| \\
& +\left\|f\left(t+h, y_{t+h}+z_{t+h}\right)-f\left(t, y_{t}+z_{t}\right)\right\| \\
& +\int_{0}^{t}\|R(t-s)\|\left\|g\left(s+h, y_{s+h}+z_{s+h}\right)-g\left(s, y_{s}+z_{s}\right)\right\| d s \\
& +\int_{0}^{h}\|R(t+h-s)\|\left\|g\left(s, x_{s}\right)\right\| d s \\
\leq & C_{1} h+L_{f}\left(h+\left\|y_{t+h}+z_{t+h}-y_{t}-z_{t}\right\|_{\mathcal{B}}\right) \\
& +M \int_{0}^{t} L_{g}\left(h+\left\|y_{s+h}+z_{s+h}-y_{s}-z_{s}\right\|_{\mathcal{B}}\right) d s+M\left\|g\left(\theta, x_{\theta}\right)\right\|_{b} h \\
\leq & C_{2} h+L_{f}\left\|z_{t+h}-z_{t}\right\|_{\mathcal{B}}+M L_{g} \int_{0}^{t}\left\|z_{s+h}-z_{s}\right\|_{\mathcal{B}} d s \\
\leq & C_{2} h+M_{b} L_{f}\left\|z_{h}\right\|_{\mathcal{B}}+K_{b} L_{f}\|z(\theta+h)-z(\theta)\|_{t} \\
& +M L_{g} M_{b}\left\|z_{h}\right\|_{\mathcal{B}}+M L_{g} K_{b} \int_{0}^{t}\|z(\theta+h)-z(\theta)\|_{s} d s,
\end{aligned}
$$

onde $C_{i}, i=1,2$ são constantes independentes de $t$ e $h$. Tomando supremo em $[0, t]$ e usando que $K_{b} L_{f}<1$, vemos que existem constantes $C_{i}, i=3,4,5$, independentes de $t$ e $h$ tais que

$$
\|z(\theta+h)-z(\theta)\|_{t} \leq C_{3} h+C_{4}\|z(\theta)\|_{h}+C_{5} \int_{0}^{t}\|z(\theta+h)-z(\theta)\|_{s} d s .
$$


Estudaremos agora o termo $\|z(\theta)\|_{h}$. Veja que para $s \in[0, h]$,

$$
\begin{aligned}
\|z(s)\| \leq & \|R(s) f(0, \varphi)-f(0, \varphi)\|+\left\|f(0, \varphi)-f\left(s, y_{s}+z_{s}\right)\right\| \\
& +\int_{0}^{s}\left\|R(s-\tau) g\left(\tau, y_{\tau}+z_{\tau}\right)\right\| d \tau \\
\leq & C_{6} h+L_{f}\left\|y_{s}-\varphi\right\|_{\mathcal{B}}+L_{f}\left\|z_{s}\right\|_{\mathcal{B}}+M\left\|g\left(\theta, y_{\theta}+z_{\theta}\right)\right\|_{b} h \\
\leq & C_{7} h+L_{f}\left\|z_{s}\right\|_{\mathcal{B}} \\
\leq & C_{7} h+L_{f} K_{b}\|z(\theta)\|_{s},
\end{aligned}
$$

de onde concluímos a existência de $C_{8}>0$ independente de $t$ e $h$ tal que

$$
\|z(\theta)\|_{h} \leq C_{8} h
$$

pois $L_{f} K_{b}<1$. O que da desigualdade de Gronwall nos permite concluir que $z(\cdot)$ é Lipschitz sobre $[0, b]$ e como consequência que $x(\cdot)$ é Lipschitz sobre $[0, b]$. Finalmente da desigualdade

$$
\left\|x_{t+h}-x_{t}\right\|_{\mathcal{B}} \leq\left\|y_{t+h}-y_{t}\right\|_{\mathcal{B}}+K_{a}\|z(\theta+h)-z(\theta)\|_{t}+M_{a} K_{a}\|z(\theta)\|_{h},
$$

e de 2.16) obtemos que $t \rightarrow x_{t}$ é Lipschitz. A prova está completa.

Nosso primeiro resultado de regularidade será obtido assumindo que $X$ possui a propriedade de Radon-Nikodým. Para isto, dado um intervalo $J \in \mathbb{R}$ e um espaço de Banach $Z$, definimos por $W^{1,1}(J: Z)$ o seguinte espaço de funções.

$W^{1,1}(J: Z)=\left\{f \in L^{1}(J, Z)\right.$ e $f(s)=f\left(s_{0}\right)+\int_{s_{0}}^{s} g(s) d s$, para algum $s_{0} \in J$ e $\left.g \in L^{1}(J, Z)\right\}$.

Definição 2.2.2 Um espaço de Banach $Z$ tem a propriedade de Radon-Nikodým com respeito a um espaço de medida finita $(\Omega, \Sigma, \mu)$, se para cada medida vetorial $\mu$-contínua de variação limitada $G: \Sigma \rightarrow Z$ existe $g \in L^{1}(\mu: Z)$ tal que $G(E)=\int_{E} g d \mu$ para todo $E \in \Sigma$. Dizemos que $Z$ possui a propriedade de Radon-Nikodým, no que segue (RNP) se $Z$ tem a propriedade de Radon-Nikodým com respeito a todo espaço de medida finita.

Lema 2.2.3 [20] Se $Z$ e um espaço Banach que possui a (RNP) e $w:[0, a] \rightarrow X$ é uma função Lipchitz, então $w \in W^{1,1}([0, a]: X)$.

Teorema 2.2.1 Suponha que $X$ possui a (RNP). Assuma também as hipóteses do Lema 2.2.2 são satisfeitas e que $\varphi(0)+f(0, \varphi) \in D(A)$. Se $x(\cdot)$ é uma solução fraca de 2.1)-2.2) sobre $[0, b]$, então $x(\cdot)$ é uma solução semi-clássica de 2.1)-(2.2) em $[0, b]$. 
Demonstração: Do Lema 2.2.2 sabemos que as funções $x(\cdot)$ e $t \rightarrow x_{t}$ são Lipschitz sobre $[0, b]$, o que por sua vez implica que $t \rightarrow g\left(t, x_{t}\right)$ e $t \rightarrow f\left(t, x_{t}\right)$ são Lipschitz sobre $[0, b]$. Pelo Lema 2.2 .3 segue que $x(\cdot), t \rightarrow g\left(t, x_{t}\right)$ e $t \rightarrow f\left(t, x_{t}\right) \in W^{1,1}([0, a]: X)$. Seja $w(\cdot)$ a solução fraca de

$$
\begin{aligned}
\frac{d w(t)}{d t} & =A w(t)+\int_{0}^{t} B(t-s) w(s) d s+g\left(t, x_{t}\right), \quad t \in(0, b), \\
w(0) & =\varphi(0)+f(0, \varphi) .
\end{aligned}
$$

Pelo Teorema 1.2 .5 segue que $w \in C([0, b): D(A)) \cap C^{1}((0, b): X)$ e que $w(\cdot)$ verifica a equação sobre $[0, b)$. Agora, da unicidade das soluções fracas de (2.18)-(2.19) podemos concluir que $w(t)=x(t)+f\left(t, x_{t}\right)$ para $t \in[0, b)$. Isto, junto ao fato que $t \rightarrow f\left(t, x_{t}\right) \in$ $W^{1,1}([0, b]: X)$ permite concluir a prova de que $x(\cdot)$ é uma solução semi-clássica.

\subsubsection{Existência de solução clássica}

Nesta seção estabelecemos condições suficientes para que uma solução fraca de (2.1)(2.2) seja uma solução clássica.

Definição 2.2.3 Uma função $x:(-\infty, b) \rightarrow X$ é uma solução clássica de (2.1)-(2.2) sobre $[0, b)$ se $x_{0}=\varphi ; x \in C([0, b): X) \cap C^{1}((0, b): X) ; x(t) \in D(A)$ para todo $t \in[0, b)$ e 2.1) é satisfeita em $(0, b)$.

Para continuar introduzimos algumas notações que serão usadas durante o restante deste trabalho. Sejam $\left(Y,\|\cdot\|_{Y}\right),\left(Z,\|\cdot\|_{Z}\right)$ espaços de Banach. Para uma função Frechet diferenciável $\xi:[\alpha, \beta] \times Y \rightarrow Z$ denotaremos por $D_{1} \xi(t, x)$ a derivada em relação a primeira variável $t$ e por $D_{2} \xi(t, x)$ a derivada em relação a segunda variável $x$. Alêm disso, usaremos a decomposição.

$$
\xi\left(t_{0}+t, x_{0}+x\right)-\xi\left(t_{0}, x_{0}\right)=D_{1} \xi\left(t_{0}, x_{0}\right) t+D_{2} \xi\left(t_{0}, x_{0}\right) x+\|(t, x)\| r\left(\xi, t_{0}, x_{0}, t, x\right)
$$

onde $r\left(\xi, t_{0}, x_{0}, 0,0\right)=0$ e $\left\|r\left(\xi, t_{0}, x_{0}, t, x\right)\right\|_{Z} \rightarrow 0$ quando $\|(t, x)\|=|t|+\|x\|_{Y} \rightarrow 0$.

No que segue, para uma função $j:[0, a] \rightarrow Z$ denotaremos por $\partial_{h} j:[0, a] \rightarrow Z$ a função definida por

$$
\partial_{h} j(t)=\frac{j(t+h)-j(t)}{h}, \quad h \in \mathbb{R} .
$$


Adicionalmente, para $x \in X$ usaremos a notação $\chi_{x}$ para a função $\chi_{x}:(-\infty, 0] \rightarrow X$ definida por

$$
\chi_{x}(\theta)= \begin{cases}x & \text { se } \quad \theta=0, \\ 0 & \text { se } \quad \theta<0 .\end{cases}
$$

O seguinte Lema será importante na prova do Teorema 2.2.2. Para não estender o texto omitiremos a demonstração.

Lema 2.2.4 Seja $K \subset J \times \Lambda$ compacto e $\Lambda \subset Y$ aberto. Se $\xi: J \times \Lambda \rightarrow Z$ é uma função conti- nuamente Frechet diferenciável, então para todo $\epsilon>0$, existe $\delta>0$ tal que $\left\|r\left(\xi, t_{0}, x_{0}, t, x\right)\right\|_{Z}<\epsilon$ para todo $\left(t_{0}, x_{0}\right) \in K$ quando $\|(t, x)\|<\delta$.

No próximo resultado estabelecemos a existência de solução clássica para 2.1 - 2.2 usando a usual hipótese de $f$ e $g$ serem funções diferenciaveis.

Teorema 2.2.2 Assuma que a função $S(\cdot) \varphi$ é Lipschitz sobre $[0, a]$ e que $\varphi(0) \in D(A)$. Suponha também que funções $f$ e $g$ são de classe $C^{1}$ sobre $[0, a] \times \mathcal{B} ; f([0, a] \times \mathcal{B}) \subset D(A)$; $D f(0, \varphi) \equiv 0$ e que $W(\cdot) \varphi$ possui derivada a direita em $t=0$. Se $g(0, \varphi) \equiv A f(0, \varphi) \equiv 0$ ou se $\chi_{g(0, \varphi)}$ e $\chi_{A f(0, \varphi)} \in \mathcal{B}$ e $\mathcal{B}$ verifica o axioma (C3), então existe uma solução clássica de (2.1)-2.2) sobre $[0, b]$ para algum $0<b \leq a$.

Demonstração: Usando que $f, g \in C^{1}([0, a] \times \mathcal{B}: X)$ e que $D(f(0, \varphi)) \equiv 0$, do Teorema 2.1.1 deduzimos a existência de uma única solução fraca $x \in C([0, b]: X)$ de 2.1)-2.2 para algum $0<b \leq a$. Mais ainda, usando novamente o fato que $D f(0, \varphi) \equiv 0$, podemos assumir que f é Lipschitz em uma vizinhança de $(0, \varphi)$ com constante $L_{f}$ tal que $L_{f} K_{b}<1$. Agora como conseqüência do Lema 2.2 .2 podemos também assumir que as funções $x(\cdot)$ e $s \rightarrow x_{s}$ são Lipschitz sobre $[0, b]$.

No que segue tomaremos $b>0$ de forma que

$$
\mu=K_{b}\left(\left\|D_{2} f\left(\theta, x_{\theta}\right)\right\|_{b}+M b\left\|D_{2} g\left(\theta, x_{\theta}\right)\right\|_{b}\right)<1 .
$$

Considere agora a equação integral,

$$
\begin{aligned}
z(t)= & R(t)[A(\varphi(0)+f(0, \varphi))+g(0, \varphi)]+\int_{0}^{t} R(t-s) B(s)(\varphi(0)+f(0, \varphi)) d s \\
& -D_{1} f\left(t, x_{t}\right)-D_{2} f\left(t, x_{t}\right) z_{t}+\int_{0}^{t} R(t-s)\left(D_{1} g\left(s, x_{s}\right)+D_{2} g\left(s, x_{s}\right) z_{s}\right) d s, \quad t \in[0, b],
\end{aligned}
$$


$\operatorname{com} z_{0}=\psi+\chi_{A f(0, \varphi)}+\chi_{g(0, \varphi)}$, onde $\psi=\left.\frac{d}{d t} W(t) \varphi\right|_{t=0}$.

Fazendo uso de (2.21) e do princípio da contração, é possível mostrar a existência de uma única solução $z(\cdot) \in C([0, b]: X)$ de $(2.22)$. Como a prova é usual será omitida.

Agora mostraremos que $x^{\prime}(\cdot)=z(\cdot)$ em $[0, b]$. Para $t \in[0, b)$ e $0<h<1$ suficientemente pequeno de modo que $t+h \in[0, b]$ temos que

$$
\begin{aligned}
& \left\|\partial_{h} x(t)-z(t)\right\| \\
& \leq \quad\left\|\partial_{h} R(t)(\varphi(0)+f(0, \varphi))-R(t)(A(\varphi(0)+f(0, \varphi)))-\int_{0}^{t} R(t-s) B(s)(\varphi(0)+f(0, \varphi)) d s\right\| \\
& \quad+\left\|\partial_{h} f\left(t, x_{t}\right)-D_{1} f\left(t, x_{t}\right)-D_{2} f\left(t, x_{t}\right) z_{t}\right\| \\
& \quad+\left\|\int_{0}^{t} R(t-s)\left(\partial_{h} g\left(s, x_{s}\right)-D_{1} g\left(s, x_{s}\right)-D_{2} g\left(s, x_{s}\right) z_{s}\right) d s\right\| \\
& \quad+\left\|\frac{1}{h} \int_{0}^{h} R(t+h-s) g\left(s, x_{s}\right) d s-R(t) g(0, \varphi)\right\|=\sum_{1}^{4} I_{i}(t, h) .
\end{aligned}
$$

No que segue, mostraremos que cada termo $I_{i}(t, h)$ converge a zero uniformemente sobre $[0, b]$ quando $h \rightarrow 0$.

Como $\varphi(0)+f(0, \varphi) \in D(A)$, é fácil ver que das propriedades do operador resolvente que $I_{1}(t, h) \rightarrow 0$ uniformemente sobre $[0, b]$ quando $h \rightarrow 0$.

Usando que a família $(R(t))_{t \geq 0}$ é fortemente contínua e que a função $t \rightarrow g\left(t, x_{t}\right)$ é contínua, obtemos que $I_{4}(t, h) \rightarrow 0$ uniformemente sobre $[0, b]$ quando $h \rightarrow 0$.

Estudaremos agora o termo $I_{2}(t, h)$. Usando a decomposição introduzida em 2.20 vemos que

$I_{2}(t, h) \leq\left\|D_{2} f\left(t, x_{t}\right)\right\|\left\|\frac{x_{t+h}-x_{t}}{h}-z_{t}\right\|_{\mathcal{B}}+\frac{\left\|\left(h, x_{t+h}-x_{t}\right)\right\|}{h}\left\|r\left(f, t, x_{t}, h, x_{t+h}-x_{t}\right)\right\|$.

Como a aplicação $t \rightarrow x_{t}$ é Lipschitz, temos do Lema 2.2.4 que

$$
\frac{\left\|\left(h, x_{t+h}-x_{t}\right)\right\|}{h}\left\|r\left(f, t, x_{t}, h, x_{t+h}-x_{t}\right)\right\| \rightarrow 0,
$$

uniformemente para $t \in[0, b]$ quando $h \rightarrow 0$. Assim podemos reescrever a desigualdade anterior na forma

$$
I_{2}(t, h) \leq \Lambda_{2}(t, h)+\left\|D_{2} f\left(t, x_{t}\right)\right\|\left\|\frac{x_{t+h}-x_{t}}{h}-z_{t}\right\|_{\mathcal{B}},
$$

onde $\Lambda_{2}(t, h) \rightarrow 0$ uniformemente para $t \in[0, b]$ quando $h \rightarrow 0$.

Usando um argumento similar ao anterior é possível mostrar que

$$
I_{3}(t, h) \leq \Lambda_{3}(t, h)+M \int_{0}^{t}\left\|D_{2} g\left(s, x_{s}\right)\right\|\left\|\frac{x_{s+h}-x_{s}}{h}-z_{s}\right\|_{\mathcal{B}} d s,
$$


onde $\Lambda_{3}(t, h) \rightarrow 0$ uniformemente para $t \in[0, b]$ quando $h \rightarrow 0$.

Dos passos anteriores, podemos reescrever $(2.23)$ na forma

$$
\begin{aligned}
\left\|\partial_{h} x(t)-z(t)\right\| \leq H(t, h) & +\left\|D_{2} f\left(t, x_{t}\right)\right\|\left\|\frac{x_{t+h}-x_{t}}{h}-z_{t}\right\|_{\mathcal{B}} \\
& +M \int_{0}^{t}\left\|D_{2} g\left(s, x_{s}\right)\right\|\left\|\frac{x_{s+h}-x_{s}}{h}-z_{s}\right\|_{\mathcal{B}} d s
\end{aligned}
$$

onde $H(t, h) \rightarrow 0$ uniformemente para $t \in[0, b]$ quando $h \rightarrow 0$. Mais ainda, dos axiomas do espaço de fase segue que

$$
\begin{aligned}
\| & \partial_{h} x(t)-z(t) \| \\
\leq \quad & H(t, h)+\left\|D_{2} f\left(t, x_{t}\right)\right\|\left(K_{b}\left\|\partial_{h} x(\theta)-z(\theta)\right\|_{t}+M_{b}\left\|\frac{x_{h}-\varphi}{h}-z_{0}\right\|_{\mathcal{B}}\right) \\
\quad & \quad \int_{0}^{t} M\left\|D_{2} g\left(s, x_{s}\right)\right\|\left(K_{b}\left\|\partial_{h} x(\theta)-z(\theta)\right\|_{s}+M_{b}\left\|\frac{x_{h}-\varphi}{h}-z_{0}\right\|_{\mathcal{B}}\right) d s \\
\leq & H(t, h)+\mu\left\|\partial_{h} x(\theta)-z(\theta)\right\|_{t}+\frac{M_{b} \mu}{K_{b}}\left\|\frac{x_{h}-\varphi}{h}-z_{0}\right\|_{\mathcal{B}} .
\end{aligned}
$$

Tomando supremo em $[0, t]$ e usando o fato de que $\mu<1$, obtemos

$$
\left\|\partial_{h} x(\theta)-z(\theta)\right\|_{t} \leq \frac{1}{1-\mu} \sup _{s \in[0, t]} H(s, h)+\frac{M_{b}}{K_{b}(1-\mu)}\left\|\frac{x_{h}-\varphi}{h}-z_{0}\right\|_{\mathcal{B}} .
$$

Conseqüentemente, para mostrar que $x^{\prime}(\cdot)=z(\cdot)$ em $[0, b]$, é suficiente provar que $\frac{x_{h}-\varphi}{h} \rightarrow z_{0}$ quando $h \rightarrow 0^{+}$. Para isto, considere a decomposição $x=z^{1}+z^{2}$ onde $\left(z^{i}\right)_{0}=0$ e

$$
\begin{aligned}
& z^{1}(\theta)=R(\theta) f(0, \varphi)-f\left(\theta, x_{\theta}\right), \quad \theta \in[0, b], \\
& z^{2}(\theta)=\int_{0}^{\theta} R(\theta-s) g\left(s, x_{s}\right) d s, \quad \theta \in[0, b] .
\end{aligned}
$$

Com estas notações temos que $x_{s}=W(s) \varphi+z_{s}^{1}+z_{s}^{2}$ e assim que

$$
\begin{aligned}
\left\|\frac{x_{h}-\varphi}{h}-z_{0}\right\|_{\mathcal{B}} & \leq\left\|\frac{W(h) \varphi+z_{h}^{1}+z_{h}^{2}-\varphi}{h}-\left(\psi+\chi_{A f(0, \varphi)}+\chi_{g(0, \varphi)}\right)\right\|_{\mathcal{B}} \\
& \leq\left\|\frac{W(h) \varphi-\varphi}{h}-\psi\right\|_{\mathcal{B}}+\left\|\frac{z_{h}^{1}}{h}-\chi_{A f(0, \varphi)}\right\|_{\mathcal{B}}+\left\|\frac{z_{h}^{2}}{h}-\chi_{g(0, \varphi)}\right\|_{\mathcal{B}} \\
& =\sum_{i=1}^{3} I_{i}(h, \mathcal{B}) .
\end{aligned}
$$

É claro que $I_{1}(h, \mathcal{B}) \rightarrow 0$ quando $h \rightarrow 0$. Suponha agora que $A f(0, \varphi) \equiv g(0, \varphi) \equiv 0$. Usando o axioma (A), vemos que

$$
\left\|\frac{z_{h}^{1}}{h}\right\|_{\mathcal{B}}+\left\|\frac{z_{h}^{2}}{h}\right\|_{\mathcal{B}} \leq K_{b}\left\|\frac{z^{1}(\theta)}{h}\right\|_{h}+K_{b}\left\|\frac{z^{2}(\theta)}{h}\right\|_{h} .
$$


Se $[x, y]$ representa o segmento de reta ligando os vetores $x, y$. Para $z^{1}$, das propriedades do operador resolvente e o fato de que $A f(0, \varphi)=0$ vemos para $\theta \in(0, h)$ que

$$
\begin{aligned}
\frac{1}{h} & \left\|z^{1}(\theta)\right\| \\
& \leq \frac{1}{h}\|R(\theta) f(0, \varphi)-f(0, \varphi)\|+\frac{1}{h}\left\|f(0, \varphi)-f\left(\theta, x_{\theta}\right)\right\| \\
& \leq \frac{1}{h}\left\|\int_{0}^{\theta} R^{\prime}(\xi) f(0, \varphi) d \xi\right\|+\frac{1}{h}\left\|f(0, \varphi)-f\left(\theta, x_{\theta}\right)\right\| \\
& \leq \frac{1}{h}\left\|\int_{0}^{\theta} \int_{0}^{\xi} R(\xi-\tau) B(\tau) f(0, \varphi) d \tau d \xi\right\|+\frac{1}{h} \sup _{(s, \zeta) \in\left[(0, \varphi),\left(\theta, x_{\theta}\right)\right]}\|D f(s, \zeta)\|\left\|\left(\theta, \varphi-x_{\theta}\right)\right\| \\
& \leq \frac{M \theta}{h} \int_{0}^{\theta}\|B(\tau) f(0, \varphi)\| d \tau+\frac{\left\|\left(\theta, \varphi-x_{\theta}\right)\right\|}{\theta} \frac{\theta}{h} \sup _{(s, \zeta) \in\left[(0, \varphi),\left(\theta, x_{\theta}\right)\right]}\|D f(s, \zeta)\|,
\end{aligned}
$$

o que mostra que $\frac{1}{h}\left\|z^{1}(\theta)\right\|_{h} \rightarrow 0$ se $h \rightarrow 0$ pois $s \rightarrow x_{s}$ é Lipschitz em $[0, b]$ e

$$
\sup _{(s, \zeta) \in\left[(0, \varphi),\left(\theta, x_{\theta}\right)\right]}\|D f(s, \zeta)\| \rightarrow 0,
$$

quando $h \rightarrow 0$. Por outro lado, para $z^{2}$ obtemos que

$$
\frac{1}{h}\left\|z^{2}(\theta)\right\|_{h} \leq \frac{1}{h} \int_{0}^{\theta}\left\|R(\theta-s) g\left(s, x_{s}\right)\right\| d s \leq \frac{M \theta}{h}\left\|g\left(\theta, x_{\theta}\right)\right\|_{h},
$$

o que prova que $\frac{1}{h}\left\|z^{2}(\theta)\right\|_{h} \rightarrow 0$ se $h \rightarrow 0$, uma vez que $\left\|g\left(\theta, x_{\theta}\right)\right\|_{h} \rightarrow 0$ quando $h \rightarrow 0$.

Assuma agora que $g(0, \varphi) \neq 0, A f(0, \varphi) \neq 0$ e que $\mathcal{B}$ verifica o axioma (C3). É fácil ver que $\left.\frac{d}{d t} z^{2}(t)\right|_{t=0^{+}}=g(0, \varphi)$, e como por hipótese $\chi_{g(0, \varphi)} \in \mathcal{B}$, temos pelo axioma (C3) que $I_{3}(h, \mathcal{B}) \rightarrow 0$ quando $h \rightarrow 0$. Em relação a $z^{1}$, veja que

$$
\begin{aligned}
\frac{z^{1}(\theta)-z^{1}(0)}{\theta}= & \frac{R(\theta) f(0, \varphi)-f(0, \varphi)}{\theta}+\frac{f(0, \varphi)-f\left(\theta, x_{\theta}\right)}{\theta} \\
= & \frac{R(\theta) f(0, \varphi)-f(0, \varphi)}{\theta}+\frac{D f(0, \varphi)\left(\theta, \varphi-x_{\theta}\right)}{\theta} \\
& +r\left(f, 0, \varphi, \theta, \varphi-x_{\theta}\right) \frac{\left\|\left(\theta, \varphi-x_{\theta}\right)\right\|}{\theta} .
\end{aligned}
$$

Como $\theta \rightarrow x_{\theta}$ é Lipschitz vemos que do Teorema 1.2 .3 que o termo da direita na última igualdade converge para $A f(0, \varphi)$, como por hipótese $\chi_{A f(0, \varphi)} \in \mathcal{B}$ usando o axioma (C3) concluímos que $I_{2}(h, \mathcal{B}) \rightarrow 0$ se $h \rightarrow 0$.

Portanto, em qualquer dos casos considerados, $A f(0, \varphi)=g(0, \varphi)=0$ ou quando $A f(0, \varphi) \neq 0$ e $g(0, \varphi) \neq 0$ e $\mathcal{B}$ satisfaz o axioma (C3), temos que $I_{i}(h, \mathcal{B}) \rightarrow 0, i=2,3$, quando $h \rightarrow 0$.

Mais ainda, é necessário observar que das estimativas anteriores obtemos que $\partial_{h} x(t) \rightarrow$ $x^{\prime}(t)$ uniformemente para $t \in[0, b]$ quando $h \rightarrow 0$. 
Agora mostraremos que a função $t \rightarrow x_{t}$ é continuamente diferenciável em $\mathcal{B}$. Para isto definimos a função $w:(-\infty, b] \rightarrow X$ por

$$
w(\theta)=\left\{\begin{aligned}
z(\theta), & \text { se } \theta \in(-\infty, 0], \\
x^{\prime}(\theta), & \text { se } \theta \in[0, b] .
\end{aligned}\right.
$$

Das etapas anteriores obtemos que $\left.\frac{d}{d t} x(t)\right|_{t=0}=\psi(0)+A f(0, \varphi)+g(0, \varphi)$, o que implica que $x^{\prime}\left(0^{+}\right)=z(0)$, assim concluímos que $w_{0}=z_{0} \in \mathcal{B}$ e $w$ é contínua em $[0, b]$, temos pelo axioma do espaço de fase (A1) que $w_{t} \in \mathcal{B}$ para todo $t \in[0, b]$ e que a aplicação $t \rightarrow w_{t}$ e contínua em $[0, b]$. Isto junto com a desigualdade

$$
\left\|\partial_{h} x_{t}-w_{t}\right\|_{\mathcal{B}} \leq M_{b}\left\|\frac{x_{h}-\varphi}{h}-z_{0}\right\|_{\mathcal{B}}+K_{b}\left\|\partial_{h} x(\theta)-x^{\prime}(\theta)\right\|_{t}
$$

mostra que $t \rightarrow x_{t}$ é continuamente diferenciável em [0, b].

Seja $v(\cdot)$ a solução fraca de

$$
\begin{aligned}
\frac{d w(t)}{d t} & =A w(t)+\int_{0}^{t} B(t-s) w(s) d s+g\left(t, x_{t}\right), \quad t \in[0, b] \\
w(0) & =\varphi(0)+f(0, \varphi) .
\end{aligned}
$$

Como a função $s \rightarrow g\left(s, x_{s}\right)$ é de classe $C^{1}$ sobre $[0, b]$ e $\varphi(0)+f(0, \varphi) \in D(A)$, do Corolário 1.2.1 obtemos que $v(\cdot)$ é uma solução clássica de 2.26)-(2.27). Da unicidade da solução fraca de 2.26)-2.27) concluímos que $v(t)=x(t)+f\left(t, x_{t}\right)$ para todo $t \in[0, b]$. Como consequência, $x(\cdot)$ verifica (2.1)-(2.2) sobre $[0, b]$ e $x(t)+f\left(t, x_{t}\right) \in D(A)$ para todo $t \in[0, b]$. Mais ainda como $f\left(t, x_{t}\right) \in D(A)$ para todo $t \in[0, b]$, segue que $x(t) \in D(A)$ para cada $t \in[0, b]$ o que finalmente prova que $x(\cdot)$ é uma solução clássica de 2.1)-2.2. A prova está completa.

\section{$2.3 \quad$ Exemplo}

Para finalizar este capítulo, nesta seção aplicamos alguns de nossos resultados abstratos. Previamente, introduzimos os elementos técnicos nessesarios. Seja $X=L^{2}([0, \pi])$. Considere o operador $A: D(A) \subset X \rightarrow X$ definido por $A f(\xi)=f^{\prime \prime}(\xi)$, onde

$$
D(A)=\left\{f \in X: f^{\prime \prime} \in X, \quad f(0)=f(\pi)=0\right\} .
$$

É conhecido que $A$ é o gerador infinitesimal de um semigrupo analítico e compacto $(T(t))_{t \geq 0}$ sobre $X$. Além disso, $A$ possui espectro discreto, com autovalores $-n^{2}, n \in \mathbb{N}$, e autovetores normalizados $z_{n}(\xi)=\left(\frac{2}{\pi}\right)^{1 / 2} \sin (n \xi)$. Além disso, as seguintes propriedades são verificadas: 
(a) $\left\{z_{n}: n \in \mathbb{N}\right\}$ é uma base ortonormal de $X$;

(b) Se $f \in D(A)$ então $A f=-\sum_{n=1}^{\infty} n^{2}<f, z_{n}>z_{n}$;

(c) Para $f \in X, T(t) f=\sum_{n=1}^{\infty} e^{-n^{2} t}<f, z_{n}>z_{n}$. Em particular, vemos que $(T(t))_{t \geq 0}$ é um semigrupo uniformemente estável com $\|T(t)\| \leq e^{-t}$ para $t \geq 0$.

No que segue usaremos como espaço de fase o espaço $\mathcal{B}=C_{r} \times L^{2}(\rho, X) \operatorname{com} r=0$, o qual foi introduzido em 1.3 .

Considere o sistema integro-diferencial

$$
\begin{aligned}
\frac{d}{d t}\left(u(t)+a_{0} * u(t)\right) & =A\left(u(t)+a_{0} * u(t)\right)+A\left(b *\left(u+a_{0} * u\right)\right)(t)+a_{1} * u(t), \\
u(t, 0) & =u(t, \pi)=0, \quad t \in[0, a] \\
u(\theta, \xi) & =\phi(\theta, \xi), \quad \theta \leq 0, \xi \in[0, \pi]
\end{aligned}
$$

No sistema anterior, $a_{0}, a_{1}: \mathbb{R} \rightarrow \mathbb{R}$ são funções contínuas, $b(t)=t^{\alpha-1} e^{-\omega t} \operatorname{com} \alpha \in(0,1) \mathrm{e}$ $\omega>0$ e

$$
\begin{aligned}
a_{0} * u(t) & =\int_{-\infty}^{t} a_{0}(t-s) u(s, \xi) d s \\
a_{1} * u(t) & =\int_{-\infty}^{t} a_{1}(t-s) u(s, \xi) d s \\
b * u(t) & =\int_{0}^{t}(t-s)^{\alpha-1} e^{-\omega(t-s)} u(s, \xi) d s
\end{aligned}
$$

Para estudar este sistema assumiremos que

$$
L_{f}:=\left(\int_{-\infty}^{0} \frac{a_{0}(-s)^{2}}{\rho(s)} d s\right)^{\frac{1}{2}}<1, \quad L_{g}:=\left(\int_{-\infty}^{0} \frac{a_{1}(-s)^{2}}{\rho(s)} d s\right)^{\frac{1}{2}}<\infty,
$$

e que a função $\varphi$ definida por $\varphi(\theta)(\xi)=\phi(\theta, \xi)$ pertence a $\mathcal{B}$.

Lema 2.3.1 Existe uma família Resolvente $(R(t))_{t \geq 0}$ associada ao sistema integro-diferencial

$$
\begin{aligned}
& x^{\prime}(t)=A x(t)+\int_{0}^{t} A b(t-s) x(s) d s, \\
& x(0)=x_{0} \in X .
\end{aligned}
$$

Demonstração: Basta observar que o operador $A$ e a família de operadores $(A b(t))_{t \geq 0}$ satisfazem as hipóteses de [24, Theorem 3.1].

Proposição 2.3.1 Nas condições anteriores, existe uma única solução fraca para o sistema 2.28)-2.29) sobre $[0, b]$ para algum $0<b \leq a$. 
Demonstração: Definamos $f, g:[0, a] \times \mathcal{B} \rightarrow X$ por

$$
\begin{aligned}
& f(t, \psi)(\xi)=\int_{-\infty}^{0} a_{0}(-s) \psi(s)(\xi) d s \\
& g(t, \psi)(\xi)=\int_{-\infty}^{0} a_{1}(-s) \psi(s)(\xi) d s
\end{aligned}
$$

Das propriedades das funções $a_{0}$ e $a_{1}$ é fácil ver que as funções $f$ e $g$ estão bem definidas. Mais ainda, $f(t, \cdot)$ e $g(t, \cdot)$ são operadores lineares contínuos com $\|f(t, \cdot)\|_{\mathcal{L}(\mathcal{B}, X)} \leq L_{f}$ e $\|g(t, \cdot)\|_{\mathcal{L}(\mathcal{B}, X)} \leq L_{g}$ para todo $t \in[0, a]$. Nestas condições, o Teorema 2.1.1 garante a existência de uma única solução fraca para o sistema (2.28)-(2.29) sobre $[0, b]$, para algum $0<b \leq a$, isto conclui a prova.

O próximo resultado é consequência direta do Teorema 2.2.1.

Corolário 2.3.1 Suponha que $R(\cdot) \varphi(0), S(\cdot) \varphi$ e $R(\cdot) f(0, \varphi)$ são Lipschitz sobre [0,a]. Se $\varphi(0)+f(0, \varphi) \in D(A)$, então existe uma solução semi-clássica de 2.28)-2.29) em algum intervalo $[0, b]$ com $0<b \leq a$. 


\title{
Capítulo 3
}

\section{Existência e regularidade de}

\author{
soluções para um sistema
}

\section{integro-diferencial do tipo neutro}

\begin{abstract}
Resumo
Neste capítulo estudaremos a existência de soluções fracas, semi-clássicas e clássicas para uma equação integro-diferencial do tipo neutro com retardamento não limitado modelada na forma

$$
\begin{aligned}
\frac{d}{d t}\left(x(t)+F\left(t, x_{t}\right)\right) & =A x(t)+\int_{0}^{t} B(t-s) x(s) d s+G\left(t, x_{t}\right), \quad t \in(0, a), \\
x_{0} & =\varphi \in \Omega \subset \mathcal{B}
\end{aligned}
$$

onde $A: D(A) \subset X \rightarrow X, B(t): D(B(t)) \subset X \rightarrow X, t \geq 0$ são operadores fechados em $X ; \mathcal{B}$ é um espaço de fase definido de maneira axiomática; $\Omega \subset \mathcal{B}$ é aberto e $F, G: I \times \mathcal{B} \rightarrow$ $Y_{i}, i=1,2$ são funções apropriadas.
\end{abstract}

\subsection{Existência de soluções fracas}

Nesta seção usaremos a teoria de operadores resolventes e vários resultados de ponto fixo no estudo da existência de soluções fracas, semi-clássicas e clássicas (conceitos a serem introduzidos), para o sistema neutro (3.1)-(3.2). Neste capítulo, assumiremos que $(R(t))_{t \geq 0}$ é uma família resolvente associada ao sistema (1.1)-(1.2); que os operadores $A, B(t), t \geq 0$, e 
$(R(t))_{t \geq 0}$ comutam entre si quando $x \in D(A)$ e que $M>0$ é tal que $\|R(t)\| \leq M$ para todo $t \in[0, a]$. Como no capítulo anterior, $y:(-\infty, a] \rightarrow X$ é a função definida por

$$
y(t)=\left\{\begin{array}{rll}
R(t) \varphi(0) & \text { se } & 0 \leq t \leq a, \\
\varphi(t) & \text { se } & -\infty<t<0 .
\end{array}\right.
$$

Lembrando que para uma função limitada $\xi:[0, b] \rightarrow[0, \infty)$ e $t \in[0, b]$, usaremos a notação $\xi_{t}$ para $\xi_{t}=\sup \{\xi(s): s \in[0, t]\}$. E para uma função contínua $\eta:[0, b] \rightarrow Z$ usaremos a notação $\|\eta(\theta)\|_{t}=\sup \{\|\eta(\theta)\|: \theta \in[0, t]\}$.

Para começarmos introduzimos o conceito de solução fraca para o sistema (3.1)-(3.2).

Definição 3.1.1 Uma função $x:(-\infty, b] \rightarrow X, 0<b \leq a$, é uma solução fraca de 3.1)(3.2) sobre $[0, b]$ se $x_{0}=\varphi ; x \in C([0, b]: X)$; as funções $s \rightarrow A R(t-s) F\left(s, x_{s}\right)$ e $s \rightarrow$ $\int_{0}^{s} B(s-\xi) R(t-s) F\left(\xi, x_{\xi}\right) d \xi$ são integráveis sobre $[0, t)$ para todo $t \in[0, b] e$

$$
\begin{aligned}
x(t) & =R(t)(\varphi(0)+F(0, \varphi))-F\left(t, x_{t}\right)-\int_{0}^{t} A R(t-s) F\left(s, x_{s}\right) d s \\
& -\int_{0}^{t} \int_{0}^{s} B(s-\xi) R(t-s) F\left(\xi, x_{\xi}\right) d \xi d s+\int_{0}^{t} R(t-s) G\left(s, x_{s}\right) d s, \quad t \in[0, b] .
\end{aligned}
$$

Antes de continuar, é necessário observar que o sistema (3.1)-3.2 apresenta uma dificuldade maior que o sistema 2.1)-2.2 tratado no capítulo anterior. A diferença entre os dois sistemas tem origem no conceito de solução fraca. Observe que de modo geral, a menos do caso trivial em que $A$ é um operador limitado, a função $s \rightarrow A R(t-s)$ não é integrável em $[0, t), t>0$, na topologia uniforme de operadores, e que uma situação similar acontece com a função $s \rightarrow B(t-s)$. Assim, no estudo do sistema (3.1)-3.2 é necessário introduzir alguma hipótese (sobre F, por exemplo) de modo de garantir a integrabilidade das funções $s \rightarrow A R(t-s) F\left(s, u_{s}\right)$ e $\tau \rightarrow B(s-\tau) R(t-s) F\left(\tau, u_{\tau}\right)$ que aparecem na definição de solução fraca.

Motivados pelo comentário anterior, introduzimos o seguinte esquema técnico. Nesta seção $\left(Y_{i} ;\|\cdot\|_{Y_{i}}\right), i=1,2$ serão espaços de Banach continuamente incluídos em $X$ e assumiremos que a seguinte hipótese é verificada.

(H) Para cada $t>0$, e todo $s \in[0, a], R(t) \in \mathcal{L}\left(X, Y_{i}\right), A R(t) \in \mathcal{L}\left(Y_{1}, X\right)$ e $B(s) R(t) \in$ $\mathcal{L}\left(Y_{2}, X\right)$. Mais ainda, as seguintes propriedades são válidas.

(i) Para todo $x \in Y_{1}$ e todo $t>0, A R(\cdot) x \in C((0, a]: X)$. Para todo $x \in Y_{2}$ e todo $t>0, B(\cdot) R(t) x \in L^{1}([0, a]: X)$. 
(ii) Existem funções $\mathcal{H}_{i}(\cdot), a(\cdot) \in L^{1}([0, a])$ tais que

$$
\begin{aligned}
\|A R(t)\|_{\mathcal{L}\left(Y_{1}, X\right)} & \leq \mathcal{H}_{1}(t), \quad t \in(0, a], \\
\|B(s) R(t)\|_{\mathcal{L}\left(Y_{2}, X\right)} & \leq a(s) \mathcal{H}_{2}(t), \quad s \in[0, a], \quad t>0 .
\end{aligned}
$$

Observação 3.1.1 É interessante justificar a condição $\left(\mathbf{H}_{\mathbf{1}}\right)$. Como mencionamos previamente, as funções $s \rightarrow A R(s), s \rightarrow B(s)$ não são integraveis de $[0, a]$ em $\mathcal{L}(X)$. Se $\left(\mathbf{H}_{\mathbf{1}}\right)$ é válida, $u \in C\left([0, a]: Y_{1}\right)$ e $v \in C\left([0, a]: Y_{2}\right)$, então do criterio de Bochner para funções integráveis e das estimativas

$$
\begin{aligned}
\|A R(t-s) u(s)\| & \leq\|A R(t-s)\|_{\mathcal{L}\left(Y_{1}, X\right)}\|u(s)\|_{Y_{1}} \leq \mathcal{H}_{1}(t-s)\|u(s)\|_{Y_{1}}, \\
\|B(s-\tau) R(\mu) y(\tau)\| & \leq\|B(s-\tau) R(\mu)\|_{\mathcal{L}\left(Y_{2}, X\right)}\|y(\tau)\|_{Y_{2}} \\
& \leq a(s-\tau) \mathcal{H}_{2}(\mu)\|y(\tau)\|_{Y_{2}}, \quad \mu>0,
\end{aligned}
$$

segue que $s \rightarrow A R(t-s) x(s) e \tau \rightarrow B(s-\tau) R(\mu) y(\tau)$ são integráveis sobre $[0, t) 0<t<a$, o que é essencial em nosso objetivo de estudar a existência de soluções fracas por meio da técnica de ponto fixo. Este tipo de condições e verificada em vários casos. Se $(R(t))_{t \geq 0}$ é um semigrupo de operadores, veja Rankin [64, Theorem 2], Lunardi [55] e Pazy [63, Theorem 6.13]. Para o caso geral, considere Grimmer [25, Theorem 3.4].

Estamos agora em condições de estabelecer nosso primeiro resultado de existência de solução fraca. Para isto usaremos o Princípio da Contração.

Teorema 3.1.1 Seja $\varphi \in \Omega$ e suponha que a condição $\left(\mathbf{H}_{\mathbf{1}}\right)$ é verificada. Assuma também que as seguintes condições são válidas.

(a) A função $F$ assume valores em $Y_{i}, i=1,2 ; F: \mathbb{R} \times \Omega \rightarrow Y_{i}, i=1,2$ é contínua e existe uma constante $L_{F}>0$ tal que

$$
\left\|F\left(t, \psi_{1}\right)-F\left(t, \psi_{2}\right)\right\|_{Y_{i}} \leq L_{F}\left\|\psi_{1}-\psi_{2}\right\|_{\mathcal{B}}, \quad \psi_{j} \in \Omega, \quad i, j=1,2, \quad t \in[0, a]
$$

(b) A função $G: \mathbb{R} \times \Omega \rightarrow X$ é contínua e existe uma constante $L_{G}>0$ tal que

$$
\left\|G\left(t, \psi_{1}\right)-G\left(t, \psi_{2}\right)\right\| \leq L_{G}\left\|\psi_{1}-\psi_{2}\right\|_{\mathcal{B}}, \quad \psi_{j} \in \Omega, \quad j=1,2, \quad t \in[0, a] .
$$

Se $L_{F}\|I\|_{\mathcal{L}\left(Y_{1}, X\right)} K(0)<1$, então existe uma única solução fraca de (3.1)-(3.2) sobre $[0, b]$ para algum $0<b \leq a$. 
Demonstração: Fixemos constantes $b_{\varphi}, r_{1}, C_{F}$ e $C_{G}$ tais que $B_{r_{1}}(\varphi, \mathcal{B}) \subseteq \Omega,\|F(t, \psi)\|_{Y_{i}} \leq$ $C_{F}, i=1,2$, e $\|G(t, \psi)\| \leq C_{G}$, para todo $t \in\left[0, b_{\varphi}\right]$ e $\psi \in B_{r_{1}}(\varphi, \mathcal{B})$. Usando a continuidade das funções $t \rightarrow y_{t}$ e $K(\cdot)$, escolhemos $\rho>0$ e $0<b_{1}<b_{\varphi}$ de forma que

$$
\begin{aligned}
\mu=L_{F}\|I\|_{\mathcal{L}\left(Y_{1}, X\right)} K_{b_{1}} & <1 \\
K_{b_{1}} \rho+\sup _{t \in\left[0, b_{1}\right]}\left\|y_{t}-\varphi\right\|_{\mathcal{B}} & <r_{1} .
\end{aligned}
$$

Seja $0<b \leq b_{1}$ tal que as seguintes desigualdades sejam validas.

$$
\begin{aligned}
\left\|(R(\theta)-I) F\left(\theta, y_{\theta}\right)\right\|_{b} & \leq \frac{(1-\mu) \rho}{3}, \\
M\left\|F(0, \varphi)-F\left(\theta, y_{\theta}\right)\right\|_{b} & \leq \frac{(1-\mu) \rho}{3}, \\
C_{F} \int_{0}^{b} \mathcal{H}_{1}(s) d s+C_{F} \int_{0}^{b} \mathcal{H}_{2}(s) d s \int_{0}^{b} a(\tau) d \tau+b M C_{G} & \leq \frac{(1-\mu) \rho}{3}, \\
K_{b}\left(L_{F} \int_{0}^{b} \mathcal{H}_{1}(s) d s+L_{F} \int_{0}^{b} \mathcal{H}_{2}(s) d s \int_{0}^{b} a(\tau) d \tau+b M L_{G}\right) & <\frac{1-\mu}{2} .
\end{aligned}
$$

Sobre o espaço $S(b)=\left\{u:(-\infty, b] \rightarrow X:\left.u\right|_{[0, b]} \in C([0, b], X), u_{0}=0,\|u(\theta)\|_{b} \leq \rho\right\}$, munido da norma da convergência uniforme, definimos $\Gamma: S(b) \rightarrow C((-\infty, b]: X)$ por $(\Gamma x)_{0}=0 \mathrm{e}$

$$
\begin{aligned}
\Gamma x(t) & =R(t) F(0, \varphi)-F\left(t, x_{t}+y_{t}\right)-\int_{0}^{t} A R(t-s) F\left(s, x_{s}+y_{s}\right) d s \\
& -\int_{0}^{t} \int_{0}^{s} B(s-\xi) R(t-s) F\left(\xi, x_{\xi}+y_{\xi}\right) d \xi d s+\int_{0}^{t} R(t-s) G\left(s, x_{s}+y_{s}\right) d s, t \in[0, b] .
\end{aligned}
$$

Nas próximas linhas mostraremos que $\Gamma$ é uma contração de $S(b)$ em $S(b)$. Vejamos inicialmente que $\Gamma$ está corretamente definida. Se $x \in S(b)$, por 3.6 temos que

$$
\left\|x_{t}+y_{t}-\varphi\right\|_{\mathcal{B}} \leq\left\|x_{t}\right\|_{\mathcal{B}}+\left\|y_{t}-\varphi\right\|_{\mathcal{B}} \leq K_{b_{1}} \rho+\sup _{t \in\left[0, b_{1}\right]}\left\|y_{t}-\varphi\right\|_{\mathcal{B}}<r_{1}
$$

Assim, $x_{t}+y_{t} \in B_{r_{1}}(\varphi, \mathcal{B})$ para todo $t \in[0, b]$ e conseqüentemente $\left\|F\left(s, x_{s}+y_{s}\right)\right\|_{Y_{i}} \leq C_{F}$, $i=1,2$ e $\left\|G\left(s, x_{s}+y_{s}\right)\right\| \leq C_{G}$ para todo $s \in[0, b]$. Logo,

$$
\begin{aligned}
\left\|A R(t-s) F\left(s, x_{s}+y_{s}\right)\right\| & \leq\|A R(t-s)\|_{\mathcal{L}\left(Y_{1}, X\right)}\left\|F\left(s, x_{s}+y_{s}\right)\right\|_{Y_{1}} \\
& \leq \mathcal{H}_{1}(t-s) C_{F}, \\
\left\|B(s-\tau) R(t-s) F\left(\tau, x_{\tau}+y_{\tau}\right)\right\| & \leq\|B(s-\tau) R(t-s)\|_{\mathcal{L}\left(Y_{2}, X\right)}\left\|F\left(\tau, x_{\tau}+y_{\tau}\right)\right\|_{Y_{2}} \\
& \leq a(s-\tau) \mathcal{H}_{2}(t-s) C_{F}, \\
\left\|R(t-s) G\left(s, x_{s}+y_{s}\right)\right\| & \leq\|R(t-s)\|_{\mathcal{L}(X, X)}\left\|G\left(s, x_{s}+y_{s}\right)\right\| \leq M C_{G},
\end{aligned}
$$


o que do Teorema de integração de Bochner, veja [20, Theorem 2], nos permite concluir que as funções $s \rightarrow A R(t-s) F\left(s, x_{s}+y_{s}\right), s \rightarrow R(t-s) G\left(s, x_{s}+y_{s}\right)$ e $\tau \rightarrow B(s-\tau) R(t-s) F\left(\tau, x_{\tau}+y_{\tau}\right)$ são integráveis sobre $[0, t)$ e $[0, s)$ respectivamente. Isto motra que $\Gamma x$ está corretamente definida e que $\Gamma x \in C((-\infty, b]: X)$.

Mostramos agora que $\Gamma S(b) \subset S(b)$. Se $x \in S(b)$ e $t \in[0, b]$, vemos que

$$
\begin{aligned}
\| & \Gamma x(t) \| \\
\leq & \left\|R(t)\left(F(0, \varphi)-F\left(t, y_{t}\right)\right)\right\|+\left\|R(t) F\left(t, y_{t}\right)-F\left(t, y_{t}\right)\right\| \\
& +\left\|F\left(t, y_{t}\right)-F\left(t, x_{t}+y_{t}\right)\right\|+\int_{0}^{t}\left\|A R(t-s) F\left(s, x_{s}+y_{s}\right)\right\| d s \\
& \quad+\int_{0}^{t} \int_{0}^{s}\left\|B(s-\xi) R(t-s) F\left(\xi, x_{\xi}+y_{\xi}\right)\right\| d \xi d s+\int_{0}^{t}\left\|R(t-s) G\left(s, x_{s}+y_{s}\right)\right\| d s \\
\leq & M\left\|F(0, \varphi)-F\left(\theta, y_{\theta}\right)\right\|_{b}+\left\|R(t) F\left(\theta, y_{\theta}\right)-F\left(\theta, y_{\theta}\right)\right\|_{b} \\
& +\|I\|_{\mathcal{L}\left(Y_{1}, X\right)} L_{F}\left\|x_{t}\right\|_{\mathcal{B}}+C_{F} \int_{0}^{b} \mathcal{H}_{1}(s) d s+C_{F} \int_{0}^{b} \mathcal{H}_{2}(s) d s \int_{0}^{b} a(s) d s+M C_{G} b \\
\leq & \frac{(1-\mu) \rho}{3}+\frac{(1-\mu) \rho}{3}+\|I\|_{\mathcal{L}\left(Y_{1}, X\right)} L_{F} K_{b}\|x(\theta)\|_{b}+\frac{(1-\mu) \rho}{3} \\
\leq & (1-\mu) \rho+\mu \rho=\rho .
\end{aligned}
$$

Como consequencia $\|\Gamma x(t)\| \leq \rho$ para todo $t \in[0, b]$ o que implica que $\Gamma S(b) \subset S(b)$.

Vejamos finalmente que $\Gamma$ é uma contração. Se $u, v \in S(b)$, então

$$
\begin{aligned}
\| \Gamma u(t) & -\Gamma v(t) \| \\
\leq \quad & F\left(t, u_{t}+y_{t}\right)-F\left(t, v_{t}+y_{t}\right) \| \\
& +\int_{0}^{t}\|A R(t-s)\|_{\mathcal{L}\left(Y_{1}, X\right)}\left\|F\left(s, u_{s}+y_{s}\right)-F\left(s, v_{s}+y_{s}\right)\right\|_{Y_{1}} d s \\
& +\int_{0}^{t} \int_{0}^{s}\|B(s-\xi) R(t-s)\|_{\mathcal{L}\left(Y_{2}, X\right)}\left\|F\left(\xi, u_{\xi}+y_{\xi}\right)-F\left(\xi, v_{\xi}+y_{\xi}\right)\right\|_{Y_{2}} d \xi d s \\
& +\int_{0}^{t} M\left\|G\left(s, u_{s}+y_{s}\right)-G\left(s, v_{s}+y_{s}\right)\right\| d s \\
\leq \quad & I\left\|_{\mathcal{L}\left(Y_{1}, X\right)} L_{F} K_{b}\right\| u(\theta)-v(\theta) \|_{b}+\left(L_{F} K_{b} \int_{0}^{b} \mathcal{H}_{1}(t-s)\|u(\theta)-v(\theta)\|_{b} d s\right. \\
& +L_{F} K_{b} \int_{0}^{b} \mathcal{H}_{2}(s) d s \int_{0}^{b} a(s) d s\|u(\theta)-v(\theta)\|_{b} d s \\
& \left.+M K_{b} \int_{0}^{b} L_{G}\|u(\theta)-v(\theta)\|_{b} d s\right),
\end{aligned}
$$

de onde obtemos que

$$
\|\Gamma u(\theta)-\Gamma v(\theta)\|_{b} \leq \mu\|u(\theta)-v(\theta)\|_{b}+\frac{1-\mu}{2}\|u(\theta)-v(\theta)\|_{b} \leq\left(\frac{1}{2}+\frac{\mu}{2}\right)\|u(\theta)-v(\theta)\|_{b},
$$


o que mostra $\Gamma$ é uma contração.

Finalmente, pelo princípio da contração, $\Gamma$ possui um ponto fixo $x(\cdot) \in S(b)$. É claro que $u(\cdot)=y(\cdot)+x(\cdot)$ é a única solução fraca de (3.1)- 3.2 , o que completa a demostração.

Nos próximos resultados, estudamos a existência de soluções fracas sem assumir que $F$ ou $G$ sejam Lipschitz.

Teorema 3.1.2 Seja $\varphi \in \Omega$ e suponha que a condição $\left(\mathbf{H}_{\mathbf{1}}\right)$ é válida. Assuma também, $R(\cdot) \in C((0, b], \mathcal{L}(X))$ e que as seguintes condições são válidas.

(a) A função $F$ é tal que $F: \mathbb{R} \times \Omega \rightarrow Y_{i}, i=1,2$, é contínua e existe $L_{F}>0$ tal que

$$
\left\|F\left(t, \psi_{1}\right)-F\left(t, \psi_{2}\right)\right\|_{Y_{i}} \leq L_{F}\left\|\psi_{1}-\psi_{2}\right\|_{\mathcal{B}}, \quad \psi_{j} \in \Omega, \quad i, j=1,2, \quad t \in[0, a]
$$

(b) A função $G: \mathbb{R} \times \Omega \rightarrow X$ é contínua e existe uma constante $r_{\varphi}>0$, tal que para cada $0<t<a$ existe um compacto $W_{t} \subseteq X$ tal que $R(t) G(s, \psi) \in W_{t}$ para todo $\psi \in B_{r_{\varphi}}(\varphi, \mathcal{B})$ e todo $s \in[0, a]$.

Se $L_{F}\|I\|_{\mathcal{L}\left(Y_{1}, X\right)} K(0)<1$, então existe uma solução fraca de (3.1)-(3.2) sobre $[0, b]$ para algum $0<b \leq a$.

Demonstração: Sejam $b, S(b), \rho, r_{1}, \Gamma(\cdot)$ como na prova do Teorema 3.1.1, e assuma que $r_{1} \leq r_{\varphi}$. Considere a decomposição $\Gamma=\Gamma_{1}+\Gamma_{2}$, onde $\left(\Gamma_{i} x\right)_{0}=0, i=1,2$, e

$$
\begin{aligned}
\Gamma_{1} x(t)= & R(t) F(0, \varphi)-F\left(t, x_{t}+y_{t}\right)-\int_{0}^{t} A R(t-s) F\left(s, x_{s}+y_{s}\right) d s \\
& +\int_{0}^{t} \int_{0}^{s} B(s-\xi) R(t-s) F\left(\xi, x_{\xi}+y_{\xi}\right) d \xi d s, \quad t \in[0, a], \\
\Gamma_{2} x(t)= & \int_{0}^{t} R(t-s) G\left(s, x_{s}+y_{s}\right) d s, \quad t \in[0, a] .
\end{aligned}
$$

Da prova do Teorema 3.1.1, sabemos que $\Gamma_{1}$ é uma contração de $S(b)$ em $S(b)$. Mais ainda, uma aplicação do Lema 2.1.1 mostra que $\Gamma_{2}$ é completamente contínua.

$\mathrm{O}$ anterior, junto com Teorema 1.3.1, mostra que $\Gamma$ possui um ponto fixo $x \in S(b)$ o que completa a prova, pois $u=x+y$ é uma solução fraca de (3.1)-(3.2).

$\left(\mathbf{H}_{\mathbf{G}}\right)$ A função $G: I \times \mathcal{B} \rightarrow X$ satisfaz as seguintes condições

(i) A função $G(t, \cdot): \mathcal{B} \rightarrow X$ é contínua para quase todo $t \in[0, a]$ e $G(\cdot, x):[0, a] \rightarrow X$ é fortemente mensurável para todo $x \in X$; 
(ii) Existe uma função contínua $m_{G}:[0, a] \rightarrow[0, \infty)$ e uma função contínua e não decrescente $\Omega_{G}:[0, \infty) \rightarrow(0, \infty)$ tal que

$$
\|G(t, \psi)\| \leq m_{G}(t) \Omega_{G}\left(\|\psi\|_{\mathcal{B}}\right), \quad(t, \psi) \in[0, a] \times \mathcal{B} .
$$

$\left(\mathbf{H}_{\mathbf{F}}\right)$ A função $F:[0, a] \times \mathcal{B} \rightarrow X$ é tal que $F([0, a] \times \mathcal{B}) \subset Y_{i}, i=1,2, F:[0, a] \times \mathcal{B} \rightarrow Y_{1}$ é completamente contínua e existem constantes positivas $c_{1}, c_{2}$ tais que

$$
\|F(t, \psi)\|_{Y_{i}} \leq c_{1}\|\psi\|_{\mathcal{B}}+c_{2}, \quad i=1,2, \quad(t, \psi) \in[0, a] \times \mathcal{B}
$$

Sejam $y(\cdot)$ a função em $(3.3)$ e $S(a)=\left\{x:(-\infty, a] \rightarrow X: x_{0}=0,\left.x\right|_{[0, a]} \in C([0, a]:\right.$ $X)\}$, munido com a norma da convergência uniforme. Para todo $Q \subset S(a)$ limitado, o conjunto de funções $\left\{t \rightarrow F\left(t, x_{t}+y_{t}\right): x \in Q\right\}$ é equicontínuo sobre $[0, a]$.

Teorema 3.1.3 Seja $\varphi \in \mathcal{B}$ e assuma que as condições $\left(\mathbf{H}_{\mathbf{1}}\right),\left(\mathbf{H}_{\mathbf{G}}\right)$ e $\left(\mathbf{H}_{\mathbf{F}}\right)$ são verificadas. Suponha também que $A R(\cdot) \in C\left((0, a]: \mathcal{L}\left(Y_{1}, X\right)\right), R(\cdot) \in C((0, a]: \mathcal{L}(X))$, a constante $0<\mu=K(0)\|I\|_{\mathcal{L}\left(Y_{1}, X\right)} c_{1}<1$ e que as seguintes propriedades são válidas.

(a) Para todo $t \in(0, a]$ e todo $r>0$, existem conjuntos compactos $W_{r}^{i}(t) \subset X, i=1,2$, tais que $B(s) R(t) F(s, \psi) \in W_{r}^{1}(t)$ e $R(t) G(s, \psi) \in W_{r}^{2}(t)$ para todo $s \in[0, a]$ e todo $\psi \in B_{r}(0, \mathcal{B})$

(b) Existe uma função positiva $\beta \in L^{1}([0, a])$ tal que para todo $c \in(0, a]$ existe uma função positiva $W_{c} \in C([0, a])$ com $W_{c}(0)=0$ tal que

$$
\left\|B(\tau) R(s)-B(\tau) R\left(s^{\prime}\right)\right\|_{\mathcal{L}\left(Y_{2}, X\right)} \leq \beta(\tau) W_{c}\left(\left|s-s^{\prime}\right|\right), \quad \tau \in[0, a], s, s^{\prime} \in[c, a] .
$$

Então existe uma solução fraca de (3.1)-(3.2) sobre $[0, b]$ para algum $0<b \leq a$.

Demonstração: Seja $0<b \leq a$ tal que

$$
\begin{array}{r}
\frac{K_{a} M}{\left(1-\mu_{1}\right)} \int_{0}^{b} m_{G}(s) d s<\int_{C}^{\infty} \frac{d s}{\Omega_{G}(s)}, \\
c_{1} K_{b}\left(\|I\|_{\mathcal{L}\left(Y_{1}, X\right)}+\int_{0}^{b} \mathcal{H}_{1}(s) d s+\int_{0}^{b} \mathcal{H}_{2}(s) d s \int_{0}^{b} a(\tau) d \tau\right)=\mu_{1}<\mu+\frac{(1-\mu)}{2},
\end{array}
$$

onde

$$
\begin{aligned}
& C=\left(M_{a}+K_{a} M H\right)\|\varphi\|_{\mathcal{B}}+\frac{K_{a} M}{1-\mu_{1}}\|F(0, \varphi)\| \\
& +\frac{K_{a} c_{2}+K_{a}\left(M_{a}+K_{a} M H\right) c_{1}\|\varphi\|_{\mathcal{B}}}{1-\mu_{1}}\left(\|I\|_{\mathcal{L}\left(Y_{1}, X\right)}+\int_{0}^{a} \mathcal{H}_{1}(s) d s+\int_{0}^{a} \mathcal{H}_{2}(s) d s \int_{0}^{a} a(s) d s\right) .
\end{aligned}
$$


Sobre o espaço $S(b)=\left\{u:(-\infty, b] \rightarrow X:\left.u\right|_{[0, b]} \in C([0, b], X), u_{0}=0\right\}$, munido da norma da convergência uniforme, definimos o operador $\Gamma: S(b) \rightarrow S(b)$ como na prova do Teorema 3.1.1. Usando os mesmos argumentos da prova do Teorema 3.1.1 segue que $\Gamma x \in S(b)$.

Mostraremos agora que $\Gamma$ verifica as condições do Teorema 1.3.2. Vejamos inicialmente que $\Gamma$ é contínua. Seja $\left(x^{n}\right)_{n \in \mathbb{N}}$ uma seqüência em $S(b)$ covergente a $x(\cdot) \in S(b)$. Dos axiomas do espaço de fase, vemos que o conjunto $U=\left\{x_{s}^{n}+y_{s}, x_{s}+y_{s}: s \in[0, b], n \in \mathbb{N}\right\}$ é relativamente compacto em $\mathcal{B}$ e que existe $r>0$ tal que $\left\|x_{s}^{n}+y_{s}\right\|_{\mathcal{B}}<r$ para todo $s \in[0, b]$ e todo $n \in \mathbb{N}$. Como $F:[0, b] \times \mathcal{B} \rightarrow X$ é contínua e $[0, b] \times U$ é relativamente compacto em $[0, b] \times \mathcal{B}$, segue que $F(\cdot)$ é uniformemente contínua sobre $[0, b] \times U$ e como conseqüência, que $F\left(s, x_{s}^{n}+y_{s}\right) \rightarrow F\left(s, x_{s}+y_{s}\right)$ uniformemente sobre [0,b] quando $n \rightarrow \infty$. Por outro lado, das propriedades de $F(\cdot)$ e $G(\cdot)$ temos que

$$
\begin{aligned}
A R(t-s) F\left(s, x_{s}^{n}+y_{s}\right) & \rightarrow A R(t-s) F\left(s, x_{s}+y_{s}\right), \\
B(s-\xi) R(t-s) F\left(\xi, x_{\xi}^{n}+y_{\xi}\right) & \rightarrow B(s-\xi) R(t-s) F\left(\xi, x_{\xi}+y_{\xi}\right), \\
G\left(s, x_{s}^{n}+y_{s}\right) & \rightarrow G\left(s, x_{s}+y_{s}\right),
\end{aligned}
$$

para quase todo $0 \leq \xi<s<t$ quando $n \rightarrow \infty$. Dos fatos anteriores e do Teorema da convergência dominada de Lebesgue concluímos que $\Gamma x^{n} \rightarrow \Gamma x$ em $S(b)$, o que prova que $\Gamma$ é contínua.

Para continuar, obteremos estimativas a priori para as soluções da equação integral $z=\lambda \Gamma z, \lambda \in(0,1)$. Seja $x^{\lambda} \in S(b)$ uma solução de $z=\lambda \Gamma z, \lambda \in(0,1)$. Se $\alpha^{\lambda}$ é a função definida por $\alpha^{\lambda}(t)=\left\|x^{\lambda}(\theta)\right\|_{t}$, então para $t \in[0, b]$ vemos que

$$
\begin{aligned}
\left\|x^{\lambda}(t)\right\| \leq & \|R(t) F(0, \varphi)\|+\left\|F\left(t, x_{t}^{\lambda}+y_{t}\right)\right\|+\int_{0}^{t}\left\|A R(t-s) F\left(s, x_{s}^{\lambda}+y_{s}\right)\right\| d s \\
& +\int_{0}^{t} \int_{0}^{s}\left\|B(s-\xi) R(t-s) F\left(\xi, x_{\xi}^{\lambda}+y_{\xi}\right)\right\| d \xi d s+\int_{0}^{t}\left\|R(t-s) G\left(s, x_{s}^{\lambda}+y_{s}\right)\right\| d s \\
\leq & M\|F(0, \varphi)\|+\|I\|_{\mathcal{L}\left(Y_{1}, X\right)}\left(c_{1}\left\|x_{t}^{\lambda}+y_{t}\right\|_{\mathcal{B}}+c_{2}\right) \\
& +\int_{0}^{t} \mathcal{H}_{1}(t-s)\left(c_{1}\left\|x_{s}^{\lambda}+y_{s}\right\|_{\mathcal{B}}+c_{2}\right) d s \\
& +\int_{0}^{t} \int_{0}^{s} a(s-\xi) \mathcal{H}_{2}(t-s)\left(c_{1}\left\|x_{\xi}^{\lambda}+y_{\xi}\right\|_{\mathcal{B}}+c_{2}\right) d \xi d s \\
& +\int_{0}^{t} M m_{G}(s) \Omega_{G}\left(\left\|x_{s}^{\lambda}+y_{s}\right\|_{\mathcal{B}}\right) d s \\
\leq & M\|F(0, \varphi)\|+\|I\|_{\mathcal{L}\left(Y_{1}, X\right)} c_{1} K_{b} \alpha^{\lambda}(t)+\|I\|_{\mathcal{L}\left(Y_{1}, X\right)} c_{1}\left(M_{b}+K_{b} M H\right)\|\varphi\|_{\mathcal{B}}
\end{aligned}
$$




$$
\begin{aligned}
& +\|I\|_{\mathcal{L}\left(Y_{1}, X\right)} c_{2}+c_{1} K_{b} \alpha^{\lambda}(t) \int_{0}^{b} \mathcal{H}_{1}(s) d s+c_{1}\left(M_{b}+K_{b} M H\right)\|\varphi\|_{\mathcal{B}} \int_{0}^{b} \mathcal{H}_{1}(s) d s \\
& +c_{2} \int_{0}^{b} \mathcal{H}_{1}(s) d s+c_{1} K_{b} \alpha^{\lambda}(t) \int_{0}^{b} \mathcal{H}_{2}(s) d s \int_{0}^{b} a(s) d s \\
& +c_{1}\left(M_{b}+K_{b} M H\right)\|\varphi\|_{\mathcal{B}} \int_{0}^{b} \mathcal{H}_{2}(s) d s \int_{0}^{b} a(s) d s+c_{2} \int_{0}^{b} \mathcal{H}_{2}(s) d s \int_{0}^{b} a(s) d s \\
& +\int_{0}^{t} M m_{G}(s) \Omega_{G}\left(K_{a} \alpha^{\lambda}(s)+\left(M_{a}+K_{a} M H\right)\|\varphi\|_{\mathcal{B}}\right) d s \\
& \leq \quad M F(0, \varphi) \|+c_{2}\left(\|I\|_{\mathcal{L}\left(Y_{1}, X\right)}+\int_{0}^{b} \mathcal{H}_{1}(s) d s+\int_{0}^{b} \mathcal{H}_{2}(s) d s \int_{0}^{b} a(s) d s\right) \\
& +c_{1}\left(M_{b}+K_{b} M H\right)\|\varphi\|_{\mathcal{B}}\left(\|I\|_{\mathcal{L}\left(Y_{1}, X\right)}+\int_{0}^{b} \mathcal{H}_{1}(s) d s+\int_{0}^{b} \mathcal{H}_{2}(s) d s \int_{0}^{b} a(s) d s\right) \\
& +\mu_{1} \alpha^{\lambda}(t)+M \int_{0}^{t} m_{G}(s) \Omega_{G}\left(K_{a} \alpha^{\lambda}(s)+\left(M_{a}+K_{a} M H\right)\|\varphi\|_{\mathcal{B}) d s,}\right.
\end{aligned}
$$

e assim obtemos que

$$
\begin{aligned}
\alpha^{\lambda}(t) \leq & \frac{1}{1-\mu_{1}}\left(M\|F(0, \varphi)\|+c_{2}\left(\|I\|_{\mathcal{L}\left(Y_{1}, X\right)}+\int_{0}^{b} \mathcal{H}_{1}(s) d s+\int_{0}^{b} \mathcal{H}_{2}(s) d s \int_{0}^{b} a(s) d s\right)\right) \\
& +\frac{\left(M_{b}+K_{b} M H\right) c_{1}\|\varphi\|_{\mathcal{B}}}{1-\mu_{1}}\left(\|I\|_{\mathcal{L}\left(Y_{1}, X\right)}+\int_{0}^{b} \mathcal{H}_{1}(s) d s+\int_{0}^{b} \mathcal{H}_{2}(s) d s \int_{0}^{b} a(s) d s\right) \\
& \left.+\frac{M}{1-\mu_{1}} \int_{0}^{t} m_{G}(s) \Omega_{G}\left(K_{a} \alpha^{\lambda}(s)+\left(M_{a}+K_{a} M H\right)\|\varphi\|_{\mathcal{B}}\right) d s\right) .
\end{aligned}
$$

Definindo $\beta^{\lambda}(t)=K_{a} \alpha^{\lambda}(t)+\left(M_{a}+K_{a} M H\right)\|\varphi\|_{\mathcal{B}}$, vemos que

$$
\begin{aligned}
\beta^{\lambda}(t) \leq & \left(M_{a}+K_{a} M H\right)\|\varphi\|_{\mathcal{B}}+\frac{K_{a}}{1-\mu_{1}}\left(M\|F(0, \varphi)\|+c_{2}\left(\|I\|_{\mathcal{L}\left(Y_{1}, X\right)}+\int_{0}^{a} \mathcal{H}_{1}(s) d s\right.\right. \\
& \left.\left.+\int_{0}^{a} \mathcal{H}_{2}(s) d s \int_{0}^{a} a(s) d s\right)\right) \\
& +\frac{K_{a}\left(M_{a}+K_{a} M H\right) c_{1}\|\varphi\|_{\mathcal{B}}}{1-\mu_{1}}\left(\|I\|_{\mathcal{L}\left(Y_{1}, X\right)}+\int_{0}^{a} \mathcal{H}_{1}(s) d s+\int_{0}^{a} \mathcal{H}_{2}(s) d s \int_{0}^{a} a(s) d s\right) \\
& \left.+\frac{K_{a} M}{1-\mu_{1}} \int_{0}^{t} m_{G}(s) \Omega_{G}\left(\beta^{\lambda}(s)\right) d s\right) .
\end{aligned}
$$

Denotando por $\gamma_{\lambda}(t)$ o lado direiro da desigualdade anterior, segue que

$$
\gamma_{\lambda}^{\prime}(t) \leq \frac{K_{a} M}{1-\mu_{1}} m_{G}(t) \Omega_{G}\left(\gamma_{\lambda}(t)\right)
$$

e então,

$$
\int_{C}^{\gamma_{\lambda}(t)} \frac{d s}{\Omega_{G}(s)}<\frac{K_{a} M}{\left(1-\mu_{1}\right)} \int_{0}^{b} m_{G}(s) d s<\int_{C}^{\infty} \frac{d s}{\Omega_{G}(s)},
$$

de onde concluímos que o conjunto $\left\{\gamma_{\lambda}(\cdot): \lambda \in(0,1)\right\}$ é limitado em $C([0, b]: X)$ e assim que $\left\{x^{\lambda}(\cdot): \lambda \in(0,1)\right\}$ é limitado em $C([0, b]: X)$. 
Para completar a prova, mostraremos que $\Gamma$ é um operador compacto. Para isto introduzimos a decomposição $\Gamma=\Gamma_{1}+\Gamma_{2}$ onde $\Gamma_{1}, \Gamma_{2}: S(b) \rightarrow S(b)$ são tais que $\left(\Gamma_{i} x\right)_{0}=$ $0, i=1,2 \mathrm{e}$

$$
\begin{array}{rlrl}
\Gamma_{1} x(t)= & R(t) F(0, \varphi)-F\left(t, x_{t}+y_{t}\right)-\int_{0}^{t} A R(t-s) F\left(s, x_{s}+y_{s}\right) d s \\
& +\int_{0}^{t} R(t-s) G\left(s, x_{s}+y_{s}\right) d s, & t \in[0, b], \\
\Gamma_{2} x(t)= & \int_{0}^{t} \int_{0}^{s} B(s-\xi) R(t-s) F\left(\xi, x_{\xi}+y_{\xi}\right) d \xi d s, & t \in[0, b] .
\end{array}
$$

Usando o Lema 2.1.1 podemos deduzir facilmente que o operador $\Gamma_{1}$ é compacto. Mais ainda, usando o mesmo Lema vemos que $\Gamma_{2}$ é também compacto. Embora mostraremos com detalhes a compacidade de $\Gamma_{2}$. No que segue $B_{r}=B_{r}(0, S(b))$.

Passo 1: $\mathrm{O}$ conjunto $\Gamma_{2} B_{r}$ e equicontínuo sobre $[0, b]$.

Seja $0<\epsilon<t<b$ e $h>0$ tal que $t+h \in[0, b]$. Das hipóteses sabemos que existe $0<\delta<\epsilon$ tal que $W_{\frac{\epsilon}{2}}(s) \leq \epsilon$ para todo $0<s \leq \delta$. Nestas condições, para $0<h<\delta$ vemos que

$$
\begin{aligned}
&\left\|\Gamma_{2} u(t+h)-\Gamma_{2} u(t)\right\| \\
& \leq \int_{0}^{t-\epsilon} \int_{0}^{s}\left\|B(s-\xi)(R(t+h-s)-R(t-s)) F\left(\xi, u_{\xi}+y_{\xi}\right)\right\| d \xi d s \\
&+\int_{t-\epsilon}^{t} \int_{0}^{s}\left\|B(s-\xi)(R(t+h-s)-R(t-s)) F\left(\xi, u_{\xi}+y_{\xi}\right)\right\| d \xi d s \\
&+\int_{t}^{t+h} \int_{0}^{s}\left\|B(s-\xi) R(t+h-s) F\left(\xi, u_{\xi}+y_{\xi}\right)\right\| d \xi d s \\
& \leq \epsilon b\left\|F\left(\theta, u_{\theta}+y_{\theta}\right)\right\|_{Y_{2}, b} \int_{0}^{s} \beta(\theta) d \theta \\
&+\left\|F\left(\theta, u_{\theta}+y_{\theta}\right)\right\|_{Y_{2}, b} \int_{t-\epsilon}^{t}\left(\mathcal{H}_{2}(t+h-s)+\mathcal{H}_{2}(t-s)\right) d s \int_{0}^{b} a(\theta) d \theta \\
&+\left\|F\left(\theta, u_{\theta}+y_{\theta}\right)\right\|_{Y_{2}, b} \int_{t}^{t+h} \mathcal{H}_{2}(t+h-s) d s \int_{0}^{b} a(\theta) d \theta
\end{aligned}
$$

de onde podemos concluir que $\Gamma_{2} B_{r}$ é equicontínuo em $t$ pela direita. Estimativas similares permitem mostrar que $\Gamma_{2} B_{r}$ é equicontínuo em zero e a equicontinuidade a esquerda em $t \in(0, b]$.

Passo 2: $\mathrm{O}$ conjunto $\left\{\Gamma_{2} u(t): u \in B_{r}\right\}$ é relativamente compacto.

Seja $0<\epsilon<t<a$. Usando as hipóteses, existe $\delta>0$ tal que

$$
\left\|B(\tau) R(s)-B(\tau) R\left(s^{\prime}\right)\right\|_{\mathcal{L}\left(Y_{2}, X\right)}<\beta(\tau) W_{\epsilon}\left(\left|s-s^{\prime}\right|\right)<\beta(\tau) \epsilon
$$


para todo $\tau \in[0, b]$, e todo $s, s^{\prime} \in[\epsilon, a] \operatorname{com}\left|s-s^{\prime}\right|<\delta$. Fixemos agora pontos $0=t_{1}<$ $t_{2}, \ldots<t_{n}=t-\epsilon$, tais que $\left|t_{i}-t_{i+1}\right|<\delta$ para cada $i=1, \ldots, n$. Nestas condições, para $u \in B_{r}$ temos que

$$
\begin{aligned}
\Gamma_{2} u(t) & \\
= & \sum_{i=1}^{n-1} \int_{t_{i}}^{t_{i+1}} \int_{0}^{s} B(s-\tau)\left(R(t-s)-R\left(t-t_{i}\right)\right) F\left(\tau, u_{\tau}+y_{\tau}\right) d \tau d s \\
& +\sum_{i=1}^{n-1} \int_{t_{i}}^{t_{i+1}} \int_{0}^{s} B(s-\tau) R\left(t-t_{i}\right) F\left(\tau, u_{\tau}+y_{\tau}\right) d \tau d s \\
& +\int_{t-\epsilon}^{t} \int_{0}^{s} B(s-\tau) R(t-s) F\left(\tau, u_{\tau}+y_{\tau}\right) d \tau d s \\
\in & \sum_{i=1}^{n-1} \epsilon^{\star} B_{1}(0: X) \\
& +\sum_{i=1}^{n-1}\left(t_{i+1}-t_{i}\right) \overline{c o\left(\left\{\int_{0}^{s} B(s-\tau) R\left(t-t_{i}\right) F\left(\tau, u_{\tau}+y_{\tau}\right) d \tau: u_{\tau}+y_{\tau} \in B_{r^{\star}}(0, \mathcal{B}), s \in[0, t]\right\}\right)} \\
& +\lambda B_{1}(0: X) \\
\subset & \sum_{i=1}^{n-1} \epsilon^{\star} B_{1}(0: X)+\sum_{i=1}^{n-1}\left(t_{i+1}-t_{i}\right) \overline{c o\left([0, t] \times W_{r^{\star}}^{1}\left(t-t_{i}\right)\right)}+\lambda B_{1}(0: X),
\end{aligned}
$$

onde $\lambda=\left\|F\left(\theta, u_{\theta}+y_{\theta}\right)\right\|_{Y_{2}, b} \int_{0}^{\epsilon} \mathcal{H}_{2}(s) d s \int_{0}^{b} a(\theta) d \theta, \epsilon^{\star}=\epsilon\left(\delta\left\|F\left(\theta, u_{\theta}+y_{\theta}\right)\right\|_{Y_{2}, b} \int_{0}^{b} \beta(\tau) d \tau\right)$ e $r^{\star}=K_{b} r+\left(M_{b}+K_{b} M H\right)\|\varphi\|_{\mathcal{B}}$. Como para cada $i$ o conjunto $\overline{c o\left([0, t] \times W_{r^{\star}}^{1}\left(t-t_{i}\right)\right)}$ é relativamente compacto e tando $\lambda$ como $\epsilon^{\star}$ convergem a zero quando $\epsilon \rightarrow 0$, podemos concluir que $\Gamma_{2} B(t)$ é relativamente compacto. Isto completa a prova que $\Gamma_{2}$ é completamente contínuo.

Dos passos anteriores, segue que $\Gamma$ verifica as hipóteses do Teorema 1.3.2, o que nos permite concluir que $\Gamma(\cdot)$ possui um ponto fixo $x \in S(b)$. Obviamente $u=x+y$ é solução fraca de (3.1)-3.2), o que permite concluir a prova.

\subsection{Resultados de regularidade}

Nesta seção estudaremos a regularidade das soluções fracas estudada na seção anterior. Especificamente, estabelecemos condições de modo de obter a existência de soluções clássicas e semi-clássicas. 


\subsubsection{Existência de soluções semi-clássicas}

No que segue desta seção assumiremos que as hipóteses do Teorema 3.1.1 são satisfeitas e que $x:(-\infty, b] \rightarrow X$ é uma solução fraca de (3.1)- 3.2 . Como conseqüência da prova do Teorema 3.1.1. também suporemos que $K_{b} L_{F}\|I\|_{\mathcal{L}\left(Y_{1}, X\right)}<1$.

Além do anterior, assumiremos que a seguinte propriedade de tipo Gronwall é verificada.

$\left(\mathbf{H}_{2}\right)$ Sejam $z, \alpha \in C([0, b]: \mathbb{R}), \alpha$ crescente, tais que

$$
0 \leq z(t) \leq \alpha(t)+c_{1} \int_{0}^{t}\left(\mathcal{H}_{1}(t-s)+\mathcal{H}_{2}(t-s)\right) z(s) d s+c_{2} \int_{0}^{t} z(s) d s,
$$

para todo $t \in[0, a]$. Então existe $c_{3}>0$ independente de $t$ e $\Theta \in L^{1}([0, a])$ tal que

$$
z(t) \leq \alpha(t) c_{3} \int_{0}^{t} \Theta(t-s) d s
$$

Observação 3.2.1 A motivação para a hipótese $\left(\mathbf{H}_{2}\right)$ é o fato da seguinte condição do tipo Gronwall ser verdadeira " $S e \phi:[0, a] \rightarrow[0, \infty)$ contínua e existe uma função não decrescente $\alpha:[0, a] \rightarrow \mathbb{R}^{+}$e constantes positivas $\mu_{1}, \mu_{2}$ e $\beta \in(0,1)$ tais que

$$
\phi(t) \leq \alpha(t)+\mu_{1} \int_{0}^{t} \phi(\sigma) d \sigma+\mu_{2} \int_{0}^{t} \frac{\phi(\sigma)}{(t-\sigma)^{1-\beta}} d \sigma
$$

então

$$
\phi(t) \leq \alpha(t)\left(\mu_{3}+\mu_{1} \mu_{3}^{2} \int_{0}^{t} e^{\mu_{1} \mu_{3}(t-s)} d s\right), \text { onde } \mu_{3}=\sum_{j=0}^{n-1}\left(\frac{\mu_{2} a^{\beta}}{\beta}\right)^{j} e^{\frac{\mu_{2}^{n} \Gamma(\beta)^{n} a^{n \beta}}{\Gamma(n \beta)}},
$$

e $n$ e o primeiro inteiro positivo tal que $n \beta>1$. "

A prova do fato anteior pode ser deduzida com facilidade da prova do [63, Lemma 6.7], omitiremos detalhes adicionais. Observamos que este tipo de desigualdade apareceram freqüêntemente nesta seção.

Para nosso primeiro resultado de regularidade, precisamos do seguinte Lema.

Lema 3.2.1 Assuma que a hipótese $\left(\mathbf{H}_{\mathbf{2}}\right)$ ocorra; que as funções $R(\cdot) \varphi(0)$ e $S(\cdot) \varphi$ são Lipschitz sobre $[0, b]$; que $F\left(t, x_{t}\right) \in D(A)$ para todo $t \in[0, b]$ e que o conjunto

$$
U=\left\{A R(s) F\left(t, x_{t}\right), \int_{0}^{s} B(\theta) R(t) F\left(s, x_{s}\right) d \theta: t, \theta, s \in[0, b]\right\}
$$

é limitado. Assuma também que existem constantes positivas $L_{F}$ e $L_{G}$ tais que

$$
\begin{aligned}
\left\|F\left(t, \psi_{1}\right)-F\left(s, \psi_{2}\right)\right\|_{Y_{i}} & \leq L_{F}\left(|t-s|+\left\|\psi_{1}-\psi_{2}\right\|_{\mathcal{B}}\right), i=1,2, \\
\left\|G\left(t, \psi_{1}\right)-G\left(s, \psi_{2}\right)\right\| & \leq L_{G}\left(|t-s|+\left\|\psi_{1}-\psi_{2}\right\|_{\mathcal{B}}\right),
\end{aligned}
$$


para todo $t, s \in[0, b]$ e todo $\psi_{1}, \psi_{2} \in \mathcal{B}$. Então as funções $x(\cdot)$ e $t \rightarrow x_{t}$ são Lipschitz sobre $[0, b]$.

Demonstração: Sejam $t \in[0, b)$ e $h>0$ tais que $t+h \in[0, b]$. Usando que $R(t)(\varphi(0)+$ $F(0, \varphi))$ é Lipschitz sobre $[0, b]$ e a limitação do conjunto $U$ vemos que

$$
\begin{aligned}
\| x(t & +h)-x(t) \| \\
\leq & c_{1} h+L_{F}\|I\|_{\mathcal{L}\left(Y_{1}, X\right)} M_{b}\left\|x_{h}-\varphi\right\|_{\mathcal{B}}+L_{F}\|I\|_{\mathcal{L}\left(Y_{1}, X\right)} K_{b}\|x(\theta+h)-x(\theta)\|_{t} \\
& +\int_{0}^{t}\left\|A R(t-s)\left(F\left(s+h, x_{s+h}\right)-F\left(s, x_{s}\right)\right)\right\| d s+\int_{0}^{h}\left\|A R(t+h-s) F\left(s, x_{s}\right)\right\| d s \\
& +\int_{0}^{t} \int_{0}^{s}\left\|B(s-\xi) R(t-s)\left(F\left(\xi+h, x_{\xi+h}\right)-F\left(\xi+h, x_{\xi+h}\right)\right)\right\| d \xi d s \\
& +\int_{0}^{t} \int_{0}^{h}\left\|B(s+h-\xi) R(t-s) F\left(\xi, x_{\xi}\right)\right\| d \xi d s \\
& +\int_{0}^{h} \int_{0}^{s}\left\|B(s-\xi) R(t+h-s) F\left(\xi, x_{\xi}\right)\right\| d \xi d s \\
& +\int_{0}^{t}\left\|R(t-s)\left(G\left(s+h, x_{s+h}\right)-G\left(s, x_{s}\right)\right)\right\| d s+\int_{0}^{h}\left\|R(t+h-s) G\left(s, x_{s}\right)\right\| d s \\
\leq & c_{2} h+L_{F}\|I\|_{\mathcal{L}\left(Y_{1}, X\right)} M_{b}\left\|x_{h}-\varphi\right\|+L_{F}\|I\|_{\mathcal{L}\left(Y_{1}, X\right)} K_{b}\|x(\theta+h)-x(\theta)\|_{t} \\
& +L_{F} M_{b}\left\|x_{h}-\varphi\right\|_{\mathcal{B}} \int_{0}^{t} \mathcal{H}_{1}(s) d s+L_{F} K_{b} \int_{0}^{t} \mathcal{H}_{1}(t-s)\|x(\theta+h)-x(\theta)\|_{s} d s \\
& +L_{F} M_{b} \int_{0}^{t} \int_{0}^{s} a(s-\xi) \mathcal{H}_{2}(t-s)\left\|x_{h}-\varphi\right\|_{\mathcal{B}} d \xi d s \\
& +L_{F} K_{b} \int_{0}^{t} \int_{0}^{s} a(s-\xi) \mathcal{H}_{2}(t-s)\|x(\theta+h)-x(\theta)\|_{\xi} d \xi d s \\
& +L_{G} M_{b} M b\left\|x_{h}-\varphi\right\|_{\mathcal{B}}+L_{G} M K_{b} \int_{0}^{t}\|x(\theta+h)-x(\theta)\|_{s} d s,
\end{aligned}
$$

onde $c_{1}$ e $c_{2}$ são constantes positivas independentes de $t$ e $h$. Da desigualdade anterior, deduzimos a existência de constantes positivas $c_{i}, i=3, \cdots, 6$ tais que

$$
\begin{aligned}
&\|x(t+h)-x(t)\| \\
& \leq \quad c_{3} h+c_{4}\left\|x_{h}-\varphi\right\|_{\mathcal{B}}+L_{F}\|I\|_{\mathcal{L}\left(Y_{1}, X\right)} K_{b}\|x(\theta+h)-x(\theta)\|_{t} \\
& \quad+c_{5} \int_{0}^{t}\left(\mathcal{H}_{1}(t-s)+\mathcal{H}_{2}(t-s)\right)\|x(\theta+h)-x(\theta)\|_{s} d s+c_{6} \int_{0}^{t}\|x(\theta+h)-x(\theta)\|_{s} d s .
\end{aligned}
$$

Como $K_{b} L_{F}\|I\|_{\mathcal{L}\left(Y_{1}, X\right)}<1$ e a função $\xi(t)=\|x(\theta+h)-x(\theta)\|_{t}$ é crescente, podemos reescrever a última desigualdade na forma

$$
\begin{aligned}
& \|x(\theta+h)-x(\theta)\|_{t} \\
& \quad \leq c_{7} h+c_{8}\left\|x_{h}-\varphi\right\|_{\mathcal{B}}+c_{9} \int_{0}^{t}\left(\mathcal{H}_{1}(t-s)+\mathcal{H}_{2}(t-s)\right)\|x(\theta+h)-x(\theta)\|_{s} d s
\end{aligned}
$$




$$
+c_{10} \int_{0}^{t}\|x(\theta+h)-x(\theta)\|_{s} d s
$$

e assim concluir por $\left(\mathbf{H}_{2}\right)$ que

$$
\|x(\theta+h)-x(\theta)\|_{t} \leq\left(c_{7} h+c_{8}\left\|x_{h}-\varphi\right\|_{\mathcal{B}}\right) C \int_{0}^{t} \Theta(t-s) d s .
$$

onde $C>0$ é independente de $t$. Agora é fácil ver que o Lema estará provado se $\left\|x_{h}-\varphi\right\|_{\mathcal{B}}=$ $O(h)$. Para mostrar isto considere a decomposição $x=y+u$ onde $y(\cdot)$ é a função definida em 3.3. Pelo Lema 2.2.1, sabemos que existe $L_{y}>0$ tal que $\|y(s+\mu)-y(s)\| \leq L_{y} \mu$ para todo $s \in[0, b]$, de onde obtemos que

$$
\left\|x_{h}-\varphi\right\|_{\mathcal{B}} \leq\left\|y_{h}-\varphi\right\|_{\mathcal{B}}+\left\|u_{h}\right\|_{\mathcal{B}} \leq L_{y} h+K_{b}\|u(\theta)\|_{h} .
$$

Por outro lado, para $s \in[0, h]$,

$$
\begin{aligned}
\| u(s) & \| \\
\leq & \|R(s) F(0, \varphi)-F(0, \varphi)\|+\left\|F\left(s, y_{s}+u_{s}\right)-F(0, \varphi)\right\| \\
& +\int_{0}^{s}\left\|A R(s-\xi) F\left(\xi, y_{\xi}+u_{\xi}\right)\right\| d \xi+\int_{0}^{s} \int_{0}^{\xi}\left\|B(\xi-\tau) R(s-\xi) F\left(\tau, y_{\tau}+u_{\tau}\right) d \tau\right\| d \xi \\
& +\int_{0}^{s}\left\|R(s-\xi) G\left(\xi, y_{\xi}+u_{\xi}\right)\right\| d \xi \\
\leq & c_{11} h+K_{b}\|I\|_{\mathcal{L}\left(Y_{1}, X\right)} L_{F}\|u(\theta)\|_{s}+\sup _{\xi, \theta \in[0, b]}\left\|A R(\xi) F\left(\theta, x_{\theta}\right)\right\|_{h} h \\
& +\sup _{\theta, s, t \in[0, b]}\left\|\int_{0}^{s} B(\theta) R(t) F\left(s, x_{s}\right) d \theta\right\| h+M\left\|G\left(\theta, x_{\theta}\right)\right\| h .
\end{aligned}
$$

Usando novamente que $K_{b} L_{F}\|I\|_{\mathcal{L}\left(Y_{1}, X\right)}<1$, obtemos que $\|u(\theta)\|_{h} \leq C_{1} h$ para $h>0$. Isto junto a (3.13), e 3.14 permite concluir que $x(\cdot)$ é Lipschitz.

Finalmente, da desigualdade

$$
\left\|x_{t+h}-x_{t}\right\|_{\mathcal{B}} \leq M_{a}\left\|y_{h}-\varphi\right\|_{\mathcal{B}}+M_{a} K_{a}\|u(\theta)\|_{h}+K_{a}\|x(\theta+h)-x(\theta)\|_{t}
$$

deduzimos que $t \rightarrow x_{t}$ é também Lipschitz sobre $[0, b]$. A prova está agora completa.

Introduzimos agora os conceito de solução semi-clássica para (3.1)- (3.2)

Definição 3.2.1 Uma função $u:(-\infty, b) \rightarrow X$ é uma solução semi-clássica de (3.1)-(3.2) se $u \in C([0, b]: X) ; u_{0}=\varphi ; t \rightarrow u(t)+F\left(t, u_{t}\right) \in C([0, b): D(A)) \cap C^{1}((0, b): X)$; $u \in W^{1,1}([0, b]: X)$ e a função $t \rightarrow F\left(t, u_{t}\right) \in W^{1,1}([0, b]: X)$ e 3.1) é satisfeita em $[0, b]$.

No próximo resultado estabeleceremos a existência de solução semi-clássica para (3.1)(3.2). 
Teorema 3.2.1 Assuma que as hipóteses do Lema 3.2.1 são satisfeitas. Suponha que $\varphi(0) \in$ $D(A)) ; F([0, b] \times \mathcal{B}) \subset D(A) ;$ que a função $B(\cdot) \in L^{\infty}([0, a]: \mathcal{L}([D(A)]: X))$ e que existe $L_{F}>0$ tal que

$$
\left\|A F\left(t, \psi_{1}\right)-A F\left(s, \psi_{2}\right)\right\| \leq L_{F}\left(|t-s|+\left\|\psi_{1}-\psi_{2}\right\|_{\mathcal{B}}\right),
$$

para todo $0 \leq s, t \leq b$ e $\psi_{1}, \psi_{2} \in \Omega$.

Se X satisfaz a (RNP), então $x(\cdot)$ é uma solução semi-clássica de (3.1)-(3.2) sobre $[0, b]$.

Demonstração: Dos Lemas 2.2.1 e 3.2.1 segue que as funções $v_{i}:[0, b] \rightarrow X, i=1,2$ definidas por

$$
\begin{aligned}
& v_{1}(t)=A F\left(t, x_{t}\right), \\
& v_{2}(t)=G\left(t, x_{t}\right),
\end{aligned}
$$

são Lipschitz sobre $[0, b]$ e do Lema 2.2 .3 deduzimos que $v_{i}(\cdot) \in W^{1,1}([0, b]: X)$ para $i=$ 1,2. Pelo fato de que $B(\cdot) \in L^{\infty}([0, a]: \mathcal{L}([D(A)]: X))$ é fácil ver que a função $v_{3}(t)=$ $\int_{0}^{t} B(t-s) F\left(s, x_{s}\right) d s \in W^{1,1}([0, b]: X)$. Logo do Teorema 1.2 .5 segue que o problema de Cauchy

$$
\begin{aligned}
\frac{d z(t)}{d t} & =A z(t)+\int_{0}^{t} B(t-s) z(s) d s+v_{1}(t)+v_{2}(t)+v_{3}(t), \quad t \in(0, b), \\
z(0) & =\varphi(0)+F(0, \varphi) .
\end{aligned}
$$

tem uma única solução clássica $w(\cdot) \in C([0, b): D(A)) \cap C^{1}((0, b): X)$ que é dada por

$$
w(t)=R(t)(\varphi(0)+F(0, \varphi))+\int_{0}^{t} R(t-s)\left(v_{1}(s)+v_{2}(s)+v_{3}(s)\right) d s .
$$

Agora da unicidade de soluções fracas de (3.15)-3.16), podemos concluir que $w(t)=x(t)+$ $F\left(t, x_{t}\right)$ para todo $t \in[0, b]$. Assim

$$
\begin{aligned}
\frac{d}{d t}\left(x(t)+F\left(t, x_{t}\right)\right)= & A\left(x(t)+F\left(t, x_{t}\right)\right)+\int_{0}^{t} B(t-s)\left(x(s)+F\left(s, x_{s}\right)\right)-A F\left(t, x_{t}\right) \\
& -\int_{0}^{t} B(t-s) F\left(s, x_{s}\right) d s+G\left(t, x_{t}\right) \\
= & A x(t)+\int_{0}^{t} B(t-s) x(s) d s+G\left(t, x_{t}\right),
\end{aligned}
$$

pois $F\left(s, x_{s}\right) \in D(A)$ para todo $s \in[0, b]$. Portanto $x(\cdot)$ verifica 3.1$)$ - 3.2 , o que finaliza a prova. 


\subsubsection{Existência de solução clássica}

Nesta seção estabelecemos condições suficientes para que uma solução fraca de (3.1)(3.2) seja uma solução clássica. Para começar, uma função $f: J \rightarrow Y$ é dita $\eta$-Hölder contínua, para $\eta \in(0,1)$, se existe uma constante $L>0$ tal que

$$
\|f(s)-f(t)\| \leq L|t-s|^{\eta}, \quad \forall s, t \in J .
$$

No que segue $C^{\eta}(J: Y)$ representa o espaço das funções $\eta$-Hölder contínuas.

Para nossos próximos resultados assumiremos válida a seguinte hipótese.

$\left(\mathbf{H}_{\mathbf{R}}\right)$ Existe $K>0$ e $\beta \in(0,1)$ tal que

$$
\left\|(-A)^{2} R(t)\right\|_{\mathcal{L}\left(Y_{2}, X\right)} \leq \frac{K}{t^{2-\beta}}, \quad t>0 .
$$

Para mostrar alguns de nossos resultados, é necessário fazer o estudo prévio do seguinte sistema.

$$
\begin{aligned}
& \frac{d x(t)}{d t}=A x(t)+\int_{0}^{t} B(t-s) x(s) d s+(-A) g(t), \quad 0<t<a, \\
& x(0)=0,
\end{aligned}
$$

onde $g(\cdot) \in C^{\vartheta}\left([0, a]: Y_{2}\right) \cap C([0, a]:[D(A)]), \vartheta \in(0,1)$.

Teorema 3.2.2 Sejam $\beta, \vartheta \in(0,1)$ tal que $\beta+\vartheta>1$ e a função $g(\cdot) \in C^{\vartheta}\left([0, a]: Y_{2}\right) \cap$ $C([0, a]:[D(A)])$. Se u(.) é uma solução fraca de (3.17)-(3.18), então u(.) é uma solução clássica para (3.17)-3.18.

Demonstração: Para começar, consideremos a seguinte decomposição

$$
u(t)=\int_{0}^{t}(-A) R(t-s)(g(s)-g(t)) d s+\int_{0}^{t}(-A) R(t-s) g(t) d s=w_{1}(t)+w_{2}(t) .
$$

Do Lema 1.2.1 temos que $A w_{2}(t) \in C([0, a]: X)$. Em relação a $w_{1}(\cdot)$, defina a seqüência de funções $w_{1, \epsilon} \in C([0, a]: X)$ por

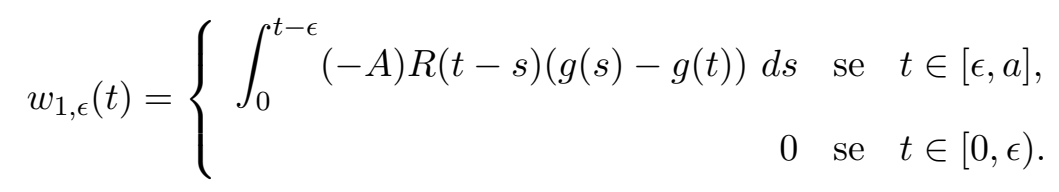

Segue da desigualdade

$$
\begin{aligned}
\left\|(-A)^{2} R(t-s)(g(s)-g(t))\right\| & \leq\left\|(-A)^{2} R(t-s)\right\|_{\mathcal{L}\left(Y_{2}, X\right)}\|g(s)-g(t)\|_{Y_{2}} \\
& \leq K L(t-s)^{\beta+\vartheta-2},
\end{aligned}
$$


que a função $s \rightarrow(-A)^{2} R(t-s)(g(s)-g(t))$ é integrável em $[0, t]$ para cada $t \in[0, a]$, segue deste fato que $w_{1, \epsilon}(\cdot) \in D(A)$. Assim podemos definir a seqüência de funções

$$
A w_{1, \epsilon}(t)=\left\{\begin{array}{rll}
\int_{0}^{t-\epsilon}(-A)^{2} R(t-s)(g(s)-g(t)) d s & \text { se } & t \in[\epsilon, a] \\
0 & \text { se } & t \in[0, \epsilon) .
\end{array}\right.
$$

É fácil ver que a sequência $w_{1, \epsilon}(t) \rightarrow \int_{0}^{t}(-A) R(t-s)(g(s)-g(t)) d s$ e que $A w_{1, \epsilon}(t) \rightarrow$ $\int_{0}^{t}(-A)^{2} R(t-s)(g(s)-g(t)) d s$ em $X$, usando que o operador $A$ é fechado concluímos que $w_{1}(t) \in D(A)$ e que $A w_{1}(t)=\int_{0}^{t}(-A)^{2} R(t-s)(g(s)-g(t)) d s$, para todo $t \in[0, a]$, portanto a função $s \rightarrow(-A) R(t-s)(g(s)-g(t)) \in L^{1}([0, a]: D(A))$. Segue do fato anterior que $w_{1}(\cdot) \in C([0, a]:[D(A)])$, e assim que $w(t) \in C([0, a]:[D(A)])$. Do Corolário 1.2.1 podemos concluir que $w(t)$ é uma solução clássica para (3.17)-(3.18). O que finaliza a prova.

Introduzimos agora o seguinte conceito de solução clássica para (3.1)-(3.2).

Definição 3.2.2 Uma função $u:(-\infty, b] \rightarrow X$ é uma solução clássica de (3.1)-(3.2) sobre $[0, b]$ se $u_{0}=\varphi ; \quad u \in C([0, b]: X) \cap C^{1}((0, b): X), u(t) \in D(A)$ para todo $t \in[0, b)$, a função $s \rightarrow B(t-s) u(s)$ é integrável em $[0, t)$ para todo $t \in[0, b)$ e 3.1] é satisfeita.

No próximo resultado estudamos a existência de solução clássica para (3.1)-(3.2).

Teorema 3.2.3 Assuma que as hipóteses $\left(\mathbf{H}_{\mathbf{1}}\right),\left(\mathbf{H}_{\mathbf{2}}\right)$ e $\left(\mathbf{H}_{\mathbf{R}}\right)$ ocorram, que $S(\cdot) \varphi$ é Lipschitz sobre $[0, a]$ e que $\varphi(0) \in D(A)$. Suponha também que

(a) $F \in C^{1}\left([0, a] \times \mathcal{B}: Y_{i}\right) \cap C([0, a] \times \mathcal{B}:[D(A)]), i=1,2$ e $D F(0, \varphi) \equiv 0$;

(b) O operador $W(\cdot) \varphi$ possui derivada a direita em $t=0$ dada por $\psi \in \mathcal{B}$;

(c) $G \in C^{1}([0, a] \times \mathcal{B}: X)$ e $G(0, \varphi) \equiv 0$ ou $\chi_{G(0, \varphi)} \in \mathcal{B}$ onde $\mathcal{B}$ satisfaz o axioma (C3);

(d) Existe $\eta \in L^{1}([0, a])$ tal que $\frac{1}{h} \int_{s}^{s+h} b(\theta) d \theta \leq \eta(s)$, para todo $h \in[0,1]$.

Então existe uma única solução clássica de (3.1)-(3.2) para algum $0<b \leq a$.

Demonstração: Usando que $F \in C^{1}\left([0, a] \times \mathcal{B}: Y_{i}\right), i=1,2, G \in C^{1}([0, a] \times \mathcal{B}: X)$ e o fato que $D(F(0, \varphi)) \equiv 0$, do Teorema 3.1.1 deduzimos a existência de uma única solução fraca $x \in C([0, b]: X)$ de 3.1 - 3.2 para algum $0<b \leq a$. Além disso, usando novamente o fato que $\operatorname{DF}(0, \varphi) \equiv 0$, podemos assumir que $F$ é Lipschitz como função de $\Omega$ em $Y_{i}, i=1,2$, 
sendo $\Omega$ aberto de $(0, \varphi)$. No que segue, $L_{F}$ será a constante de Lipschitz de $F$ em $\Omega$ e assumiremos que $L_{F} K_{b}<1$. Agora como conseqüência do Lema 3.2.1 podemos supor que as funções $x(\cdot)$ e $s \rightarrow x_{s}$ são Lipschitz sobre $[0, b]$. Mais ainda, assumiremos sem perda de generalidade que $b$ é de tal forma que

$$
\begin{aligned}
\mu= & K_{b}\left\|D_{2} F\left(s, x_{s}\right)\right\|_{Y_{1}, b}\left(\|I\|_{\mathcal{L}\left(Y_{1}, X\right)}+\int_{0}^{b} \mathcal{H}_{1}(s) d s+\int_{0}^{b} a(s) d s \int_{0}^{b} \mathcal{H}_{2}(s) d s\right) \\
& +K_{b} M b\left\|D_{2} G\left(s, x_{s}\right)\right\|_{b}<1 .
\end{aligned}
$$

Seja $z \in C([0, b]: X)$ uma solução da equação integral

$$
\begin{aligned}
z(t)= & R(t)(A \varphi(0)+G(0, \varphi))+\int_{0}^{t} R(t-s) B(s) \varphi(0) d s-D_{1} F\left(t, x_{t}\right)-D_{2} F\left(t, x_{t}\right) z_{t} \\
& +\int_{0}^{t} A R(t-s)\left(D_{1} F\left(s, x_{s}\right)+D_{2} F\left(s, x_{s}\right) z_{s}\right) d s \\
& +\int_{0}^{t} \int_{0}^{s} B(s-\xi) R(t-s)\left(D_{1} F\left(\xi, x_{\xi}\right)-D_{2} F\left(\xi, x_{\xi}\right) z_{\xi}\right) d \xi d s \\
& +\int_{0}^{t} R(t-s)\left(D_{1} G\left(s, x_{s}\right)-D_{2} G\left(s, x_{s}\right) z_{s}\right) d s
\end{aligned}
$$

com $z_{0}=\psi+\chi_{G(0, \varphi)}$. A existência de $z(\cdot)$ é conseqüência de 3.19$]$ e do princípio da contração. Omitiremos detalhes adicionais.

No que segue mostraremos que $x^{\prime}(\cdot)=z(\cdot)$ em $[0, b]$. Para $t \in[0, b]$ e $0<h<1$ suficientemente pequeno de forma que $t+h \in[0, b]$ temos que

$$
\begin{aligned}
\| \partial_{h} x( & t)-z(t) \| \\
\leq \quad & \left\|\partial_{h} R(t) \varphi(0)-R(t) A \varphi(0)-\int_{0}^{t} R(t-s) B(s) \varphi(0) d s\right\| \\
& +\left\|\partial_{h} F\left(t, x_{t}\right)-D_{1} F\left(t, x_{t}\right)-D_{2} F\left(t, x_{t}\right) z_{t}\right\| \\
& +\left\|\partial_{h} R(t) F(0, \varphi)-\frac{1}{h} \int_{0}^{h} A R(t+h-s) F\left(s, x_{s}\right) d s-\frac{1}{h} \int_{0}^{t} \int_{0}^{h} R(t-s) B(s-\xi+h) F\left(\xi, x_{\xi}\right) d \xi d s\right\| \\
& +\left\|\frac{1}{h} \int_{0}^{h} R(t+h-s) \int_{0}^{s} B(s-\xi) F\left(\xi, x_{\xi}\right) d \xi d s\right\| \\
& +\left\|\int_{0}^{t} A R(t-s)\left(\partial_{h} F\left(s, x_{s}\right)-D_{1} F\left(s, x_{s}\right)-D_{2} F\left(s, x_{s}\right) z_{s}\right) d s\right\| \\
& +\left\|\int_{0}^{t} \int_{0}^{s} B(s-\xi) R(t-s)\left(\partial_{h} F\left(\xi, x_{\xi}\right)-D_{1} F\left(\xi, x_{\xi}\right)-D_{2} F\left(\xi, x_{\xi}\right) z_{\xi}\right) d \xi d s\right\| \\
& +\left\|\int_{0}^{t} R(t-s)\left(\partial_{h} G\left(s, x_{s}\right)-D_{1} G\left(s, x_{s}\right)-D_{2} G\left(s, x_{s}\right) z_{s}\right) d s\right\| \\
& +\left\|\frac{1}{h} \int_{0}^{h} R(t+h-s) G\left(s, x_{s}\right) d s-R(t) G(0, \varphi)\right\| . \\
= & \sum_{1}^{8} I_{i}(t, h) .
\end{aligned}
$$


Analisaremos agora os termos $I_{i}(t, h), i=1, \ldots, 8$. Como $\varphi(0) \in D(A)$, das propriedades do operador resolvente, é fácil ver que $I_{1}(t, h) \rightarrow 0$ uniformemente para $t \in[0, b]$ quando $h \rightarrow 0$.

Para estudar $I_{3}(t, h)$ considere a desigualdade

$$
\begin{aligned}
I_{3}(t, h) \leq & \left\|\frac{R(t+h)-R(t)}{h} F(0, \varphi)-R(t) A F(0, \varphi)-\int_{0}^{t} R(t-s) B(s) F(0, \varphi) d s\right\| \\
& +\left\|-\frac{1}{h} \int_{0}^{t} R(t-s) \int_{0}^{h} B(s-\xi+h) F\left(\xi, x_{\xi}\right) d \xi d s+\int_{0}^{t} R(t-s) B(s) F(0, \varphi) d s\right\| \\
& +\left\|-\frac{1}{h} \int_{0}^{h} A R(t+h-s) F\left(s, x_{s}\right) d s+R(t) A F(0, \varphi)\right\| \\
= & \sum_{1}^{3} \widehat{I}_{i}(t, h) .
\end{aligned}
$$

Como $F(0, \varphi) \in D(A)$, das propriedades do operador resolvente temos que $\widehat{I}_{1}(t, h) \rightarrow 0$ uniformemente para $t \in[0, b]$ quando $h \rightarrow 0$. Pelo fato de $F \in C([0, b] \times \mathcal{B},[D(A)])$ segue que $\frac{1}{h} \int_{0}^{h} B(s-\xi+h) F\left(\xi, x_{\xi}\right) d \xi \rightarrow B(s) F(0, \varphi)$ q.t.p. sobre $[0, b]$. Além disso, como \| $\frac{1}{h} \int_{0}^{h} B(s-\xi+h) F\left(\xi, x_{\xi}\right) d \xi\left\|\leq \int_{s}^{s+h} b(\xi) d \xi\right\| F\left(\theta, x_{\theta}\right)\left\|_{[D(A)], b} \leq \eta(s)\right\| F\left(\theta, x_{\theta}\right) \|_{[D(A)], b}$ em $[0, b]$, deduzimos do Teorema da convergência dominada que $\widehat{I}_{2}(t, h) \rightarrow 0$ uniformemente para $t \in[0, b]$ quando $h \rightarrow 0$. Finalmente, $\widehat{I}_{3}(t, h) \rightarrow 0$ uniformemente quando $h \rightarrow 0$, desde que $F \in C([0, b] \times \mathcal{B},[D(A)])$. Como consequência do anterior, podemos concluir que $I_{3}(t, h) \rightarrow 0$ uniformemente para $t \in[0, b]$ quando $h \rightarrow 0$.

Da continuidade das funções $F(\cdot)$ e $G(\cdot)$, da integrabilidade da função $B(\cdot)$ e das propriedades do operador resolvente podemos deduzir facilmente que o termos $I_{4}(t, h) \rightarrow 0 \mathrm{e}$ $I_{8}(t, h) \rightarrow 0$ uniformemente para $t \in[0, b]$ quando $h \rightarrow 0$.

Usando as conclusões anteriores e a decomposição (2.20), podemos reescrever a desigualdade inicial desta prova na forma

$$
\begin{aligned}
\left\|\partial_{h} x(t)-z(t)\right\| \\
\leq \quad H(t, h)+\|I\|_{\mathcal{L}\left(Y_{1}, X\right)}\left\|D_{2} F\left(t, x_{t}\right)\right\|_{Y_{1}}\left\|\frac{x_{t+h}-x_{t}}{h}-z_{t}\right\|_{\mathcal{B}} \\
\quad+\frac{\left\|\left(h, x_{t+h}-x_{t}\right)\right\|}{h}\left\|r\left(F, t, x_{t}, h, x_{t+h}-x_{t}\right)\right\|_{Y_{1}} \\
\quad+\int_{0}^{t} \mathcal{H}_{1}(t-s)\left\|D_{2} F\left(s, x_{s}\right)\right\|_{Y_{1}}\left\|\frac{x_{s+h}-x_{s}}{h}-z_{s}\right\|_{\mathcal{B}} d s \\
\quad+\int_{0}^{t} \mathcal{H}_{1}(t-s) \frac{\left\|\left(h, x_{s+h}-x_{s}\right)\right\|}{h}\left\|r\left(F, s, x_{s}, h, x_{s+h}-x_{s}\right)\right\|_{Y_{1}} d s
\end{aligned}
$$




$$
\begin{aligned}
& +\int_{0}^{t} \int_{0}^{s} a(s-\xi) \mathcal{H}_{2}(t-s)\left\|D_{2} F\left(\xi, x_{\xi}\right)\right\|_{Y_{2}}\left\|\frac{x_{\xi+h}-x_{\xi}}{h}-z_{\xi}\right\|_{\mathcal{B}} d \xi d s \\
& +\int_{0}^{t} \int_{0}^{s} a(s-\xi) \mathcal{H}_{2}(t-s) \frac{\left\|\left(h, x_{\xi+h}-x_{\xi}\right)\right\|}{h}\left\|r\left(F, \xi, x_{\xi}, h, x_{\xi+h}-x_{\xi}\right)\right\|_{Y_{2}} d \xi d s \\
& +M \int_{0}^{t}\left\|D_{2} G\left(s, x_{s}\right)\right\|\left\|\frac{x_{s+h}-x_{s}}{h}-z_{s}\right\|_{\mathcal{B}} d s \\
& +M \int_{0}^{t} \frac{\left\|\left(h, x_{s+h}-x_{s}\right)\right\|}{h}\left\|r\left(G, s, x_{s}, h, x_{s+h}-x_{s}\right)\right\| d s
\end{aligned}
$$

onde $H(t, h) \rightarrow 0$ uniformemente para $t \in[0, b]$ quando $h \rightarrow 0$. Mais ainda, dos Lemas 2.2.4 e 3.2 .1 temos que para $P=F, G$,

$$
\frac{\left\|\left(h, x_{s+h}-x_{s}\right)\right\|}{h}\left\|r\left(P, s, x_{s}, h, x_{s+h}-x_{s}\right)\right\| \rightarrow 0
$$

uniformemente para $s \in[0, b]$ quando $h \rightarrow 0$. Isto permite reescrever a desigualdade anterior na forma

$$
\begin{aligned}
\left\|\partial_{h} x(t)-z(t)\right\| \leq & H_{1}(t, h)+\|I\|_{\mathcal{L}\left(Y_{1}, X\right)}\left\|D_{2} F\left(t, x_{t}\right)\right\|_{Y_{1}}\left\|\frac{x_{t+h}-x_{t}}{h}-z_{t}\right\|_{\mathcal{B}} \\
& +\int_{0}^{t} \mathcal{H}_{1}(t-s)\left\|D_{2} F\left(s, x_{s}\right)\right\|_{Y_{1}}\left\|\frac{x_{s+h}-x_{s}}{h}-z_{s}\right\|_{\mathcal{B}} d s \\
& +\int_{0}^{t} \int_{0}^{s} a(s-\xi) \mathcal{H}_{2}(t-s)\left\|D_{2} F\left(\xi, x_{\xi}\right)\right\|_{Y_{2}}\left\|\frac{x_{\xi+h}-x_{\xi}}{h}-z_{\xi}\right\|_{\mathcal{B}} d \xi d s \\
& +M \int_{0}^{t}\left\|D_{2} G\left(s, x_{s}\right)\right\|\left\|\frac{x_{s+h}-x_{s}}{h}-z_{s}\right\|_{\mathcal{B}} d s
\end{aligned}
$$

onde $H_{1}(t, h) \rightarrow 0$ uniformemente para $t \in[0, b]$ quando $h \rightarrow 0$.

Agora, como conseqüência da desigualdade

$$
\left\|\frac{x_{t+h}-x_{t}}{h}-z_{t}\right\|_{\mathcal{B}} \leq M_{b}\left\|\frac{x_{h}-\varphi}{h}-z_{0}\right\|_{\mathcal{B}}+K_{b}\left\|\frac{x(\theta+h)-x(\theta)}{h}-z(\theta)\right\|_{t},
$$

segue que

$$
\left\|\partial_{h} x(\theta)-z(\theta)\right\|_{t} \leq H(\theta, h)_{t}+\mu\left\|\partial_{h} x(\theta)-z(\theta)\right\|_{t}+\frac{\mu M_{b}}{K_{b}}\left\|\frac{x_{h}-\varphi}{h}-z_{0}\right\|_{\mathcal{B}}
$$

e assim que

$$
\left\|\partial_{h} x(\theta)-z(\theta)\right\|_{t} \leq \frac{1}{1-\mu} H(\theta, h)_{t}+\frac{\mu M_{b}}{(1-\mu) K_{b}}\left\|\frac{x_{h}-\varphi}{h}-z_{0}\right\|_{\mathcal{B}}
$$

Da última desigualdade, é claro que $x^{\prime}=z$ em $[0, b]$ se $\left\|\frac{x_{h}-\varphi}{h}-z_{0}\right\|_{\mathcal{B}} \rightarrow 0$ quando $h \rightarrow 0$. Para mostra este fato, introduzimos a decomposição $x_{t}=W(t) \varphi+\sum_{i=1}^{4} z_{t}^{1}$, onde 
$\left(z^{i}\right)_{0}=0$ e para $\theta \in[0, h]$

$$
\begin{aligned}
z^{1}(\theta) & =R(\theta) F(0, \varphi)-F\left(\theta, x_{\theta}\right), \\
z^{2}(\theta) & =\int_{0}^{\theta} A R(\theta-s) F\left(s, x_{s}\right) d s, \\
z^{3}(\theta) & =\int_{0}^{\theta} R(\theta-s) \int_{0}^{s} B(s-\xi) F\left(\xi, x_{\xi}\right) d \xi d s, \\
z^{4}(\theta) & =\int_{0}^{\theta} R(\theta-s) G\left(s, x_{s}\right) d s .
\end{aligned}
$$

Com as notações anteriores, segue que,

$$
\begin{aligned}
\left\|\frac{x_{h}-\varphi}{h}-z_{0}\right\|_{\mathcal{B}} & \leq\left\|\frac{W(h) \varphi-\varphi}{h}-\psi\right\|_{\mathcal{B}}+\left\|\frac{z_{h}^{1}+z_{h}^{2}+z_{h}^{3}}{h}\right\|_{\mathcal{B}}+\left\|\frac{z_{h}^{4}}{h}-\chi_{G(0, \varphi)}\right\|_{\mathcal{B}} \\
& =\sum_{i=1}^{3} I_{i}(h, \mathcal{B}) .
\end{aligned}
$$

Das hipóteses é óbvio que $I_{1}(h, \mathcal{B}) \rightarrow 0$ quando $h \rightarrow 0$. Para o termo $I_{3}(h, \mathcal{B})$, suponha primeiro que $G(0, \varphi) \equiv 0$. Se $\theta \in(0, h)$, então

$$
\left\|z^{4}(\theta)\right\| \leq \int_{0}^{\theta}\left\|R(\theta-s) G\left(s, x_{s}\right)\right\| d s \leq M \theta\left\|G\left(\theta, x_{\theta}\right)\right\|_{h},
$$

o que da desigualdade $\frac{1}{h}\left\|z_{h}^{4}\right\|_{\mathcal{B}} \leq K_{b} M_{\frac{\theta}{h}}\left\|G\left(\theta, x_{\theta}\right)\right\|_{h}$, prova que $\left\|\frac{z_{h}^{4}}{h}\right\|_{\mathcal{B}} \rightarrow 0$ quando $h \rightarrow 0$, pois $G(0, \varphi)=0$. Por outro lado, se $G(0, \varphi) \neq 0, \chi_{G(0, \varphi)} \in \mathcal{B}$ e $\mathcal{B}$ satisfaz o axioma (C3), temos portanto que $I_{3}(h, \mathcal{B}) \rightarrow 0$ quando $h \rightarrow 0$, uma vez que $\left.\frac{d}{d t} z^{4}(t)\right|_{t=0^{+}}=G(0, \varphi)$.

Para o termo $I_{2}(h, \mathcal{B})$ veja que

$$
\begin{aligned}
\| & \frac{z_{h}^{1}+z_{h}^{2}+z_{h}^{3}}{h} \|_{\mathcal{B}} \\
& \leq \frac{K_{b}}{h}\left\|R(\theta) F(0, \varphi)-F\left(\theta, x_{\theta}\right)+\int_{0}^{\theta}\left(A R(\theta-s) F\left(s, x_{s}\right) d s+R(\theta-s) \int_{0}^{s} B(s-\xi) F\left(\xi, x_{\xi}\right) d \xi\right) d s\right\|_{h} \\
& \leq \frac{K_{b}}{h}\left\|F\left(\theta, x_{\theta}\right)-F(0, \varphi)\right\|_{h} \\
& +\frac{K_{b}}{h}\left\|R(\theta) F(0, \varphi)-F(0, \varphi)+\int_{0}^{\theta}\left(A R(\theta-s) F\left(s, x_{s}\right) d s+R(\theta-s) \int_{0}^{s} B(s-\xi) F\left(\xi, x_{\xi}\right) d \xi\right) d s\right\|_{h} .
\end{aligned}
$$

Seja $\theta \in[0, h]$, como a função $s \rightarrow x_{s}$ é Lipschitz, existe $L>0$ tal que $\left\|x_{s}-\varphi\right\|_{\mathcal{B}} \leq L s$ para todo $s \in[0, b]$. Nestas condições temos que

$$
\left\|F\left(\theta, x_{\theta}\right)-F(0, \varphi)\right\| \leq\left\|\left(\theta, x_{\theta}-\varphi\right)\right\|\|I\|_{\mathcal{L}\left(Y_{1}, X\right)} \sup _{(s, \zeta) \in\left[(0, \varphi),\left(\theta, x_{\theta}\right)\right]}\|D F(s, \zeta)\|_{Y_{1}}
$$

onde $\left[(0, \varphi),\left(\theta, x_{\theta}\right)\right]$ representa o segmento de reta que liga os ponto $(0, \varphi)$ a $\left(\theta, x_{\theta}\right)$. O fato acima implica que $\frac{K_{b}}{h}\left\|F\left(\theta, x_{\theta}\right)-F(0, \varphi)\right\|_{h} \rightarrow 0$ quando $h \rightarrow 0$, pois $D F(0, \varphi)=0$. 
Usando novamente que a função $s \rightarrow x_{s}$ é Lipschitz e o fto que $R^{\prime}(\mu) F(0, \varphi)=$ $A R(\mu) F(0, \varphi)+\int_{0}^{\mu} B(\mu-s) R(s) F(0, \varphi) d s$ vemos que

$$
\begin{aligned}
\| & R(\theta) F(0, \varphi)-F(0, \varphi)+\int_{0}^{\theta} A R(\theta-s) F\left(s, x_{s}\right) d s+\int_{0}^{\theta} R(\theta-s) \int_{0}^{s} B(s-\xi) F\left(\xi, x_{\xi}\right) d \xi d s \| \\
= & \left\|\int_{\theta}^{0} R^{\prime}(\theta-s) F(0, \varphi) d s+\int_{0}^{\theta} A R(\theta-s) F\left(s, x_{s}\right) d s+\int_{0}^{\theta} R(\theta-s) \int_{0}^{s} B(s-\xi) F\left(\xi, x_{\xi}\right) d \xi d s\right\| \\
\leq & \int_{0}^{\theta}\left\|A R(\theta-s)\left(F\left(s, x_{s}\right)-F(0, \varphi)\right)\right\| d s \\
& \quad+\int_{0}^{\theta}\left\|\int_{0}^{s} B(s-\xi) R(\theta-s)\left(F\left(\xi, x_{\xi}\right)-F(0, \varphi)\right)\right\| d \xi d s \\
\leq & \int_{0}^{\theta} \mathcal{H}_{1}(\theta-s) L_{F}(1+L)|s| d s+\int_{0}^{\theta} \int_{0}^{s} a(s-\xi) \mathcal{H}_{2}(\theta-s) L_{F}(1+L)|\xi| d \xi d s \\
\leq & L_{F}(1+L) \theta \int_{0}^{h} \mathcal{H}_{1}(s) d s+L_{F}(1+L) \theta \int_{0}^{h} \mathcal{H}_{2}(s) d s \int_{0}^{b} a(\xi) d \xi,
\end{aligned}
$$

de onde inferimos que a expressão acima divida por $h$ converge a zero quando $h \rightarrow 0$, pois $\mathcal{H}_{1}$ e $\mathcal{H}_{2}$ são funções integráveis sobre $[0, b]$. Portanto $I_{2}(h, \mathcal{B}) \rightarrow 0$, quando $h \rightarrow 0$

Resumindo as conclusões anteriores obtemos que $\frac{x_{h}-\varphi}{h} \rightarrow z_{0}$ quando $h \rightarrow 0$. Logo temos que $x^{\prime}(\cdot)=z(\cdot)$, e que $x \in C^{1}([0, b]: X)$. Mais ainda, das estimativas é fácil observar que $\partial_{h} x(t) \rightarrow x^{\prime}(t)$ uniformemente para $t \in[0, b]$ quando $h \rightarrow 0$.

Agora mostraremos que a função $t \rightarrow x_{t}$ é continuamente diferenciável em $\mathcal{B}$. Para isto definimos a função $w:(-\infty, b] \rightarrow X$ por

$$
w(\theta)=\left\{\begin{aligned}
z(\theta), & \text { se } \theta \in(-\infty, 0], \\
x^{\prime}(\theta), & \text { se } \theta \in[0, b] .
\end{aligned}\right.
$$

Das etapas anteriores obtemos que $\left.\frac{d}{d t} x(t)\right|_{t=0}=\psi(0)+G(0, \varphi)$, o que implica que $x^{\prime}\left(0^{+}\right)=$ $z(0)$, assim temos que $w_{0}=z_{0} \in \mathcal{B}$ e como $w$ é contínua em $[0, b]$, segue dos axiomas do espaço de fase que $w_{t} \in \mathcal{B}$ para todo $t \in[0, b]$ e que a aplicação $t \rightarrow w_{t}$ e contínua em $[0, b]$. $\mathrm{O}$ anterior, junto com a desigualdade

$$
\left\|\partial_{h} x_{t}-w_{t}\right\|_{\mathcal{B}} \leq M_{b}\left\|\frac{x_{h}-\varphi}{h}-z_{0}\right\|_{\mathcal{B}}+K_{b}\left\|\partial_{h} x(\theta)-x^{\prime}(\theta)\right\|_{t}
$$

mostra que $t \rightarrow x_{t}$ é continuamente diferenciável sobre $[0, b]$. Ainda por cima, $\partial_{h} x_{t} \rightarrow \frac{d}{d t} x_{t}$ uniformemente para $t \in[0, b]$ quando $h \rightarrow 0$.

Considere o problema de Cauchy

$$
\begin{aligned}
\frac{d w(t)}{d t} & =A w(t)+\int_{0}^{t} B(t-s) w(s) d s-A F\left(t, x_{t}\right)-\int_{0}^{t} B(t-s) F\left(s, x_{s}\right) d s+G\left(t, x_{t}\right) \\
w(0) & =\varphi(0)+F(0, \varphi) .
\end{aligned}
$$


Dos fatos anteriores podemos concluir que $t \rightarrow G\left(t, x_{t}\right) \in C^{1}([0, b]: X)$ e que $t \rightarrow$ $F\left(t, x_{t}\right) \in C([0, b]:[D(A)]) \cap C^{\vartheta}\left([0, b]: Y_{2}\right), \vartheta=1$. Agora vamos mostrar que a função

$$
t \rightarrow \zeta(t)=\int_{0}^{t} R(t-s) \int_{0}^{s} B(s-\xi) F\left(\xi, x_{\xi}\right) d \xi d s \in C^{1}([0, b]: X) .
$$

De fato, seja

$\zeta^{\prime}(t)=\int_{0}^{t} \int_{0}^{s} B(s-\xi) R(t-s)\left(D_{1} F\left(\xi, x_{\xi}\right)+D_{2} F\left(\xi, x_{\xi}\right) \frac{d}{d \xi}\left(x_{\xi}\right)\right) d \xi d s-\int_{0}^{t} R(t-s) B(s) F(0, \varphi) d s$.

Pelas hipóteses sobre $F(\cdot)$ e do fato que a aplicação $t \rightarrow x_{t} \in C^{1}([0, b]: \mathcal{B})$, temos que a aplicação $\zeta^{\prime}(\cdot) \in C([0, b]: X)$. Assim vemos que

$$
\begin{aligned}
\| & \frac{\zeta(t+h)-\zeta(t)}{h}-\zeta^{\prime}(t) \| \\
\leq & \left\|\int_{0}^{t} \int_{0}^{s} B(s-\xi) R(t-s)\left(\partial_{h} F\left(\xi, x_{\xi}\right)-D_{1} F\left(\xi, x_{\xi}\right)-D_{2} F\left(\xi, x_{\xi}\right) \frac{d}{d \xi}\left(x_{\xi}\right)\right) d \xi d s\right\| \\
\leq & \left\|\frac{1}{h} \int_{0}^{h} R(t+h-s) \int_{0}^{s} B(s-\xi) F\left(\xi, x_{\xi}\right) d \xi d s\right\| \\
& \quad+\left\|\frac{1}{h} \int_{0}^{t} R(t-s) \int_{0}^{h} B(s+h-\xi) F\left(\xi, x_{\xi}\right) d \xi d s-\int_{0}^{t} R(t-s) B(s) F(0, \varphi) d s\right\| \\
= & \sum_{i=1}^{3} I_{i} .
\end{aligned}
$$

Por estimativas já feitas obtemos que $I_{i}, i=1,2$, convergem a zero quando $h \rightarrow 0$. Usando a decomposição 2.20$)$, vemos que

$$
\begin{aligned}
I_{1} \leq & \int_{0}^{t} \int_{0}^{s} a(s-\xi) \mathcal{H}_{2}(t-s)\left\|D_{2} F\left(\xi, x_{\xi}\right)\right\|_{Y_{2}}\left\|\frac{x_{\xi+h}-x_{\xi}}{h}-\frac{d}{d \xi}\left(x_{\xi}\right)\right\|_{\mathcal{B}} d \xi d s \\
& +\int_{0}^{t} \int_{0}^{s} a(s-\xi) \mathcal{H}_{2}(t-s) \frac{\left\|\left(h, x_{\xi+h}-x_{\xi}\right)\right\|}{h}\left\|r\left(F, \xi, x_{\xi}, h, x_{\xi+h}-x_{\xi}\right)\right\|_{Y_{2}} d \xi d s \\
\leq & \left(\left\|D_{2} F\left(\theta, x_{\theta}\right)\right\|_{Y_{2}, b} \int_{0}^{b} \mathcal{H}_{2}(s) d s \int_{0}^{b} a(s) d s\right) \sup _{\xi \in[0, b]}\left\|\frac{x_{\xi+h}-x_{\xi}}{h}-\frac{d}{d \xi}\left(x_{\xi}\right)\right\|_{\mathcal{B}} \\
& +\left((1+L) \int_{0}^{b} \mathcal{H}_{2}(s) d s \int_{0}^{b} a(s) d s\right)\left\|r\left(F, \theta, x_{\theta}, \theta, x_{\theta+h}-x_{\theta}\right)\right\|_{Y_{2}, b},
\end{aligned}
$$

o que permite concluir que $I_{1} \rightarrow 0$ quando $h \rightarrow 0$.

Como $\varphi(0)+F(0, \varphi) \in D(A)$, do Corolário 1.2.1 e do Teorema 3.2.2 temos que

$$
\begin{aligned}
w(t)= & R(t)(\varphi(0)+F(0, \varphi))-\int_{0}^{t} A R(t-s) F\left(s, x_{s}\right) d s \\
& -\int_{0}^{t} R(t-s) \int_{0}^{s} B(s-\xi) F\left(\xi, x_{\xi}\right) d \xi d s+\int_{0}^{t} R(t-s) G\left(s, x_{s}\right) d s,
\end{aligned}
$$

é uma solução clássica para (3.21). Da unicidade das soluções fracas de (3.1)-(3.2) segue que $w(t)=x(t)+F\left(t, x_{t}\right)$. Mais ainda, como $F\left(t, x_{t}\right) \in D(A)$ se $t \in[0, b]$, obtemos que 
$x(t) \in D(A)$ para todo $t \in[0, b]$ e que

$$
\begin{aligned}
\frac{d}{d t}\left(x(t)+F\left(t, x_{t}\right)\right)= & A\left(x(t)+F\left(t, x_{t}\right)\right)+\int_{0}^{t} B(t-s)\left(x(s)+F\left(s, x_{s}\right)\right)-A F\left(t, x_{t}\right) \\
& -\int_{0}^{t} B(t-s) F\left(s, x_{s}\right) d s+G\left(t, x_{t}\right) \\
= & A x(t)+\int_{0}^{t} B(t-s) x(s)+G\left(t, x_{t}\right) .
\end{aligned}
$$

O anterior completa a prova do resultado.

\subsection{Exemplo}

Nesta seção consideramos alguns exemplos de sistemas neutros que podem ser estudados fazendo uso dos nossos resultados abstratos deste capítulo. Previamente, introduzimos os elementos técnicos necessários. No que segue $X=L^{2}([0, \pi])$ e $A: D(A) \subset X \rightarrow X$ é o operador definido por $A f=f^{\prime \prime}$, onde

$$
D(A)=\left\{f \in X: f^{\prime \prime} \in X, \quad f(0)=f(\pi)=0\right\} .
$$

É conhecido que $A$ é o gerador infinitesimal de um semigrupo analítico e compacto $(T(t))_{t \geq 0}$ em $X$. Além disso, $A$ possui espectro discreto, os autovalores são $-n^{2}, n \in \mathbb{N}$ e os autovetores normalizados são $z_{n}(\xi)=\left(\frac{2}{\pi}\right)^{1 / 2} \sin (n \xi)$. Além do anterior, as seguintes propriedades são verificadas

(a) $\left\{z_{n}: n \in \mathbb{N}\right\}$ é uma base ortonormal de $X$;

(b) Se $f \in D(A)$ então $A f=-\sum_{n=1}^{\infty} n^{2}<f, z_{n}>z_{n}$;

(c) Para $f \in X, T(t) f=\sum_{n=1}^{\infty} e^{-n^{2} t}<f, z_{n}>z_{n}$. Em particular, vemos que $(T(t))_{t \geq 0}$ é um semigrupo uniformemente estável com $\|T(t)\| \leq e^{-t}$ para todo $t \geq 0$,

(d) Para cada $f \in X$ e todo $\alpha \in(0,1)$, temos que $(-A)^{-\alpha} f=\sum_{n=1}^{\infty} \frac{1}{n^{2 \alpha}}<f, z_{n}>z_{n}$. Em particular, $\left\|(-A)^{-1 / 2}\right\|=1$,

(e) Para cada $\alpha \in(0,1)$ e $f \in D\left((-A)^{\alpha}\right),(-A)^{\alpha} f=\sum_{n=1}^{\infty} n^{2 \alpha}<f, z_{n}>z_{n}$. Mais ainda, $D\left((-A)^{\alpha}\right)=\left\{f \in X: \sum_{n=1}^{\infty} n^{2 \alpha}<f, z_{n}>z_{n} \in X\right\}$.

Seja $B(t) f(\xi):=a(t) A f(\xi)$, onde $a(\cdot) \in L^{1}\left(\mathbb{R}^{+}\right)$, $a(\cdot)$ é limitada em intervalos da forma $0<t_{1} \leq t_{2}<\infty$. Assumiremos também que $\widehat{a}(\lambda)$ é absolutamente convergenta para 
$\operatorname{Re}(\lambda)>0$. Com as condições anteriores, o operador $A$ e a familía de operadores $(B(t))_{t \geq 0}$ satisfazem as hipóteses do Teorema [25, Theorem 3.1], e existe $K>0$ tal que

$$
\left\|(-A)^{\alpha} R(t) x\right\| \leq \frac{K}{t^{\alpha}}\|x\|, \quad t>0,
$$

para todo $\alpha \in(0,1)$ e $x \in X$, para este último fato, veja [25, Theorem 3.4]. Assim concluímos que existe uma família resolvente analítica $(R(t))_{t \geq 0}$ associada ao sistema 1.1 - 1.2 tal que a hipótese $\left(\mathbf{H}_{1}\right)$ seja verdadeira com $X=L^{2}([0, \pi])$ e $Y_{1}=Y_{2}=D\left((-A)^{1 / 2}\right)$.

Para estudar nossos próximos exemplos, escolhemos o espaço de fase $\mathcal{B}=C_{r} \times L^{2}(\rho, X)$ com $r=0$.

Considere o sistema integro-diferencial

$$
\begin{aligned}
\frac{\partial}{\partial t}\left(u(t, \xi)+\int_{-\infty}^{t} \int_{0}^{\pi} b(t-s, \eta, \xi) u(s, \eta) d \eta d s\right) & =\frac{\partial^{2}}{\partial \xi^{2}} u(t, \xi)+\int_{0}^{t} a(t-s) \frac{\partial^{2}}{\partial \xi^{2}} u(s, \xi) d s, \\
& +\int_{-\infty}^{t} a_{0}(s-t) u(s, \xi) d s,(t, \xi) \in[0, a] \times[0, \pi], \\
u(t, 0) & =u(t, \pi)=0, \quad t \in[0, a], \\
u(\theta, \xi) & =\phi(\theta, \xi), \quad \theta \leq 0, \xi \in[0, \pi] .
\end{aligned}
$$

onde se verificam as seguintes propriedades

(i) A função $\frac{\partial}{\partial \xi} b(\theta, \eta, \xi)$ é contínua, $b(\theta, \eta, 0)=b(\theta, \eta, \pi)=0$ para todo $(\theta, \eta)$ e

$L_{F}:=\max \left\{\int_{0}^{\pi} \int_{-\infty}^{0} \int_{0}^{\pi} \frac{\partial}{\partial \xi} b(\theta, \eta, \xi) d \eta d \theta d \xi,\left(\int_{0}^{\pi} \int_{-\infty}^{0} \int_{0}^{\pi}\left(\frac{\partial}{\partial \xi} b(\theta, \eta, \xi)\right)^{2} \rho^{-1}(\theta) d \eta d \theta d \xi\right)^{1 / 2}\right\}<\infty$.

(ii) A função $a_{0}(\cdot)$ é contínua e $L_{0}:=\left(\int_{-\infty}^{0} \frac{\left(a_{0}(s)\right)^{2}}{\rho(s)} d s\right)^{\frac{1}{2}}<\infty$.

(iii) A função $\varphi$ definida por $\varphi(\theta)(\xi)=\phi(\theta, \xi)$ pertence a $\mathcal{B}$.

Definimos as funções $F$ e $G:[0, a] \times \mathcal{B} \rightarrow X$ por

$$
\begin{aligned}
F(t, \psi)(\xi) & :=\int_{-\infty}^{0} \int_{0}^{\pi} b(s, \eta, \xi) \psi(s, \eta) d \eta d s \\
G(t, \psi)(\xi) & :=\int_{-\infty}^{0} a_{0}(s) \psi(s, \xi) d s .
\end{aligned}
$$

Assim podemos escrever o sistema $3.22-(3.23)$ na forma abstrata

$$
\begin{aligned}
\frac{d}{d t}\left(x(t)+F\left(t, x_{t}\right)\right) & =A x(t)+\int_{0}^{t} B(t-s) x(s) d s+G\left(t, x_{t}\right), \\
x_{0} & =\varphi \in \mathcal{B} .
\end{aligned}
$$


Alem disso, é fácil mostrar que $F(t, \cdot), G(t, \cdot)$ são operadores lineares contínuos com

$$
\|F(t, \cdot)\| \leq L_{F},\|G(t, \cdot)\| \leq L_{G}
$$

Mais ainda, usando a caracterização de $D\left((-A)^{\frac{1}{2}}\right)$ dada em (e), podemos mostrar após alguns cálculos extensos que $F(\mathbb{R} \times \mathcal{B}) \subset D\left((-A)^{\frac{1}{2}}\right)$ e que

$$
\left\|(-A)^{\frac{1}{2}} F(t, \psi)\right\| \leq L_{F}\|\psi\|_{\mathcal{B}}
$$

para todo $\psi \in \mathcal{B}$.

O seguinte resultado é conseqüência direta do Teorema 3.1 .1

Proposição 3.3.1 Suponha que as condições (i)-(iii) ocorram. Se $L_{F}\|I\|_{\mathcal{L}\left(Y_{1}, X\right)} K(0)<$ 1. Então existe uma única solução fraca para o sistema (3.22)-3.23) sobre [0,b] para algum $0<b \leq a$.

Se além das condições anteriores assumirmos que

(iv) A função $\frac{\partial^{2}}{\partial \xi^{2}} b(\theta, \eta, \xi)$ é contínua e

$$
\widetilde{L_{F}}:=\left(\int_{0}^{\pi} \int_{-\infty}^{0} \int_{0}^{\pi}\left(\frac{\partial^{2}}{\partial \xi^{2}} b(\theta, \eta, \xi)\right)^{2} \rho^{-1}(\theta) d \eta d \theta d \xi\right)^{1 / 2}<\infty
$$

obtemos o seguinte resultado como conseqüência do Teorema 3.2.1.

Corolário 3.3.1 Seja u(.) a solução fraca de (3.22)- 3.23) garantida pela Proposição 3.3.1. Se $R(\cdot) \varphi(0)$ e $S(\cdot) \varphi$ são Lipschitz em $[0, b]$, então u(·) é uma solução semi-clássica de (3.22)(3.23). 


\section{Capítulo 4}

\section{Famílias "N-resolventes" e aplica- ções a equações integro-diferenciais do tipo neutro}

\section{Resumo}

Neste capítulo estudamos a existência de uma família "N-resolvente" de operadores para um sistema integro-diferencial descrito na forma.

$$
\begin{aligned}
\frac{d}{d t}\left(x(t)+\int_{0}^{t} N(t-s) x(s) d s\right) & =A x(t)+\int_{0}^{t} B(t-s) x(s) d s, \quad t \in(0, a), \\
x(0) & =x_{0}
\end{aligned}
$$

onde $A: D(A) \subset X \rightarrow X, B(t): D(B(t)) \subset X \rightarrow X, t \geq 0$ são operadores lineares fechados com $D(B(t)) \supset D(A), t \geq 0$, e $(N(t))_{t \geq 0}$ é uma família de operadores lineares contínuos em X. Estudamos também propriedades qualitativas da família N-resolvente e a existência e regularidade de soluções fracas para o problema não homogêneo associado. Na seção 4.4 aplicamos a teoria desenvolvida, no estudo da existência e regularidade de soluções fracas para um sistema integro-diferencial do tipo neutro com retardamento não limitado. 


\subsection{Preliminares}

Neste seção estudamos a existência e propriedades qualitativas de uma família "Nresolvente" de operadores lineares limitados para o sistema (4.1)-(4.2). Brevemente, uma família N-resolvente é uma família de operadores em $\mathcal{L}(X)$, que se relaciona com o sistema 4.1)-4.2 de maneira similar como um semigrupo de operadores lineares se relacionam com a equação $x^{\prime}(t)=A x(t)$.

Na seqüência usaremos a notação $(H * p)$ para representar a convolução entre funções $H$ e $p$, a qual é definida por $(H * p)(t)=\int_{0}^{t} H(t-s) p(s) d s$. Adicionalmente, para um $\operatorname{Banach}\left(Z,\|\cdot\|_{Z}\right)$ e uma função $\xi:[0, \infty) \rightarrow Z$, usaremos a notação $\widehat{\xi}$ para representar a transformada de Laplace de $\xi$.

Neste capítulo assumiremos as seguintes hipóteses.

$\left(\mathbf{H N}_{\mathbf{1}}\right)$ O operador $A$ é gerador infinitesimal de um semigrupo analítico em $X$. Em particular, assumiremos que existem constantes $M>0, \theta \in(\pi / 2, \pi)$ tais que $\rho(A) \supset \Lambda_{\theta}=\{\lambda \in$ $\mathbb{C} \backslash\{0\}:|\arg (\lambda)|<\theta\}$ e $\|R(\lambda, A)\| \leq \frac{M}{|\lambda|}$ para todo $\lambda \in \Lambda_{\theta}$.

$\left(\mathbf{H N}_{\mathbf{2}}\right)(N(t))_{t>0}$ é uma família uniformemente limitada de operadores em $\mathcal{L}(X) ; N(t)(D(A)) \subset$ $D(A), A N(t) x=N(t) A x \forall x \in D(A)$ e $t>0 ; N(\cdot) \in L^{1}([0, \infty): \mathcal{L}(X)) \cap L^{1}([0, \infty):$ $\mathcal{L}([D(A)]) ; \widehat{N}(\lambda) x$ é absolutamente convergente para todo $x \in X$ e $\operatorname{Re}(\lambda)>0$. Mais ainda, existe $\alpha>0$ e uma extensão analítica de $\widehat{N}(\lambda)$ a $\Lambda_{\theta}$, que denotaremos por $\widehat{N}(\lambda)$, tal que $\|\widehat{N}(\lambda)\| \leq \frac{N}{|\lambda|^{\alpha}}$ para todo $\lambda \in \Lambda_{\theta}$.

$\left(\mathbf{H N}_{\mathbf{3}}\right)$ Para cada $t \geq 0, B(t): D(B(t)) \subset X \rightarrow X$ é um operador linear fechado com $D(A) \subset$ $D(B(t))$. Também assumiremos que $B(\cdot) x$ é uma função fortemente mensurável em $(0, \infty)$ para cada $x \in D(A)$ e que existe $b(\cdot) \in L^{1}\left(\mathbb{R}^{+}\right)$, com $\widehat{b}(\lambda)$ absolutamente convergente para $\operatorname{Re}(\lambda)>0$, tal que $\|B(t) x\| \leq b(t)\|x\|_{[D(A)]}$ para todo $t>0$ e cada $x \in D(A)$.

$\left(\mathbf{H N}_{4}\right)$ Existe um subconjunto $D \subset D\left(A^{2}\right)$ denso em $D(A)$ tal que $A(D) \subset D, \widehat{B}(\lambda)(D) \subset D$ e $A \widehat{B}(\lambda)(D)=\widehat{B}(\lambda) A(D)$ para todo $\lambda$ tal que $\operatorname{Re}(\lambda)>0$.

Observação 4.1.1 Como exemplo de um operador que verifica a hipótese $\left(\mathbf{H N}_{4}\right)$, considere A o operador Laplaciano com condições de Dirichlet, $B(t)=b(t) A$ e $D=C_{0}^{\infty}(\Omega)$. 
Exemplo 4.1.1 Em relação a condição $\left(\mathbf{H N}_{2}\right)$, considere $N(t)=e^{-\omega t} t^{\alpha-1} L: X \rightarrow X$ onde $\omega>0, \alpha \in(0,1)$ e $L$ é um operador em $\mathcal{L}(X)$ que comuta com o operador $A$ quando $x \in D(A)$. É fácil ver que $N(\cdot) \in L^{1}([0, \infty): \mathcal{L}(X)) \cap L^{1}([0, \infty): \mathcal{L}([D(A)])$. Além disso,

$$
\|\widehat{N}(\lambda) x\| \leq \frac{\Gamma(\alpha)\|L\|}{|\lambda|^{\alpha}}\|x\|
$$

para todo $x \in X$.

Definição 4.1.1 Uma família de operadores lineares $(R(t))_{t \geq 0}$ em $\mathcal{L}(X)$, é chamada operador $N$-resolvente para (4.1)-(4.2) se as seguintes propriedades são verificadas.

(a) $R(0)=I$, a função $t \rightarrow R(t) x$ é contínua sobre $[0, \infty)$ para todo $x \in X$ e existem constantes $\eta$ e $M \geq 1$ tais que $\|R(t)\| \leq M e^{\eta t}$ para todo $t \geq 0$.

(b) $R(t) D(A) \subset D(A)$ para todo $t \geq 0$ e a função $R(\cdot) x \in C([0, \infty) ;[D(A)])$ para todo $x \in D(A)$.

(c) Se $x \in D(A)$, então $R(\cdot) x \in C^{1}((0, \infty): X) ; R(\cdot) x+(N * R)(\cdot) x$ e $R(\cdot) x+(R * N)(\cdot) x$ são funções em $C^{1}([0, \infty): X) e$

$$
\begin{aligned}
\frac{d}{d t}\left(R(t) x+\int_{0}^{t} N(t-s) R(s) x d s\right) & =A R(t) x+\int_{0}^{t} B(t-s) R(s) x d s, \\
\frac{d}{d t}\left(R(t) x+\int_{0}^{t} R(t-s) N(s) x d s\right) & =R(t) A x+\int_{0}^{t} R(t-s) B(s) x d s,
\end{aligned}
$$

para todo $t \geq 0$.

\subsection{Existência de uma família N-resolvente}

Nesta seção estabelecemos condições suficientes para a existência de uma família Nresolvente para (4.1)-(4.2). Previamente, é necessário introduzir algumas notações e mostrar alguns resultados preliminares.

Denotaremos por $\rho_{F}$ o conjunto

$$
\rho_{F}=\left\{\lambda \in \mathbb{C} ;(\lambda+\lambda \widehat{N}(\lambda)-A)^{-1} \in \mathcal{L}(X)\right\},
$$

e $F: \rho_{F} \rightarrow \mathcal{L}(X)$ será o operador definido por $F(\lambda)=(\lambda+\lambda \widehat{N}(\lambda)-A)^{-1}$. Similarmente, $\rho_{G}$ será o conjunto

$$
\rho_{G}=\left\{\lambda \in \mathbb{C} ;(\lambda+\lambda \widehat{N}(\lambda)-\widehat{B}(\lambda)-A)^{-1} \in \mathcal{L}(X)\right\},
$$

e $G(\lambda): \rho_{G} \rightarrow \mathcal{L}(X)$ será o operador $G(\lambda)=(\lambda+\lambda \widehat{N}(\lambda)-\widehat{B}(\lambda)-A)^{-1}$. 
Lema 4.2.1 Assuma que as hipóteses $\left(\mathbf{H N}_{\mathbf{1}}\right)$ e $\left(\mathbf{H N}_{\mathbf{2}}\right)$ são válidas. Então existem constantes positivas $r, M_{1}, M_{2}$ tais que $\rho_{F} \supset \Lambda_{r, \theta}=\{\lambda \in \mathbb{C} \backslash\{0\}:|\lambda|>r$ e $|\arg (\lambda)|<\theta\}$,

$$
\begin{gathered}
\|F(\lambda)\| \leq \frac{M_{1}}{|\lambda|}, \quad \forall \lambda \in \Lambda_{r, \theta}, \\
F(\lambda)=R(\lambda, A)(I+\lambda \hat{N}(\lambda) R(\lambda, A))^{-1}, \quad \forall \lambda \in \Lambda_{r, \theta}, \\
\|A F(\lambda) x\| \leq \frac{M_{1}}{|\lambda|}\|x\|_{[D(A)]}, \quad \forall \lambda \in \Lambda_{r, \theta}, \quad x \in D(A), \\
\|A F(\lambda)\| \leq M_{2}, \quad \forall \lambda \in \Lambda_{r, \theta} .
\end{gathered}
$$

Demonstração: Sejam $\lambda \in \Lambda_{\theta}$ e $r=(2 N M)^{\frac{1}{\alpha}}$. Da estimativa

$$
\|\lambda \widehat{N}(\lambda) R(\lambda, A) x\| \leq \frac{N M}{|\lambda|^{\alpha}}\|x\|,
$$

temos que $\|\lambda \widehat{N}(\lambda) R(\lambda, A)\| \leq \frac{1}{2}$ para $|\lambda|>r$, o que implica que $(I+\lambda \widehat{N}(\lambda) R(\lambda, A))^{-1}$ existe e que $\left\|(I+\lambda \widehat{N}(\lambda) R(\lambda, A))^{-1}\right\| \leq 2$.

Por outro lado, para $x \in X$ temos que

$$
\begin{aligned}
(\lambda+\lambda \widehat{N}(\lambda)-A) R(\lambda, A)(I+\lambda \widehat{N}(\lambda) R(\lambda, A))^{-1} x & = \\
(I+\lambda \widehat{N}(\lambda) R(\lambda, A))(\lambda-A) R(\lambda, A)(I+\lambda \widehat{N}(\lambda) R(\lambda, A))^{-1} x & = \\
(I+\lambda \widehat{N}(\lambda) R(\lambda, A))(I+\lambda \widehat{N}(\lambda) R(\lambda, A))^{-1} x & =x,
\end{aligned}
$$

e que para $x \in D(A)$,

$$
\begin{aligned}
R(\lambda, A)(I+\lambda \widehat{N}(\lambda) R(\lambda, A))^{-1}(\lambda+\lambda \widehat{N}(\lambda)-A) x & = \\
R(\lambda, A)(I+\lambda \widehat{N}(\lambda) R(\lambda, A))^{-1}(I+\lambda \widehat{N}(\lambda) R(\lambda, A))(\lambda-A) x & = \\
R(\lambda, A)(\lambda-A) x & =x,
\end{aligned}
$$

o que permite concluir que $F(\lambda)$ existe para todo $\lambda$ em $\Lambda_{r, \theta}$, que

$$
F(\lambda)=R(\lambda, A)(I+\lambda \widehat{N}(\lambda) R(\lambda, A))^{-1}
$$

e que

$$
\|F(\lambda)\| \leq \frac{2 M}{|\lambda|}
$$

Do anterior temos que (4.5) e 4.6 são verificadas. 
Como $A$ é um operador fechado, para $x \in D(A)$ segue que

$$
A F(\lambda) x=A R(\lambda, A) \sum_{n=0}^{\infty}(-\lambda \widehat{N}(\lambda) R(\lambda, A))^{n} x=R(\lambda, A) \sum_{n=0}^{\infty}(-\lambda \widehat{N}(\lambda) R(\lambda, A))^{n} A x,
$$

de onde obtemos que

$$
\|A F(\lambda) x\| \leq \frac{2 M}{|\lambda|}\|x\|_{[D(A)]} .
$$

Finalmente, da definição de $F(\lambda)$ e das propriedades anteriores segue que

$$
\begin{aligned}
\|A F(\lambda)\| & =\left\|A R(\lambda, A)(I+\lambda \widehat{N}(\lambda) R(\lambda, A))^{-1}\right\| \\
& =\left\|\lambda R(\lambda, A)(I+\lambda \widehat{N}(\lambda) R(\lambda, A))^{-1}\right\|+\left\|(I+\lambda \widehat{N}(\lambda) R(\lambda, A))^{-1}\right\| \\
& \leq 2 M+2=M_{2},
\end{aligned}
$$

o que completa a prova.

Lema 4.2.2 Assuma que as hipóteses $\left(\mathbf{H N}_{\mathbf{1}}\right),\left(\mathbf{H N}_{\mathbf{2}}\right)$ e $\left(\mathbf{H N}_{\mathbf{3}}\right)$ são válidas. Sejam $r, M_{1}, M_{2}$ as constantes garatidas no Lema 4.2.1 e suponha que $\limsup _{|\lambda| \rightarrow \infty} \widehat{b}(\lambda) M_{2}<1$. Então existem constantes $r_{1}, M_{3}, M_{4}$ e $M_{5}$ tais que $\rho_{G} \supset \Lambda_{r_{1}, \theta}$

$$
\begin{gathered}
\|G(\lambda)\| \leq \frac{M_{3}}{|\lambda|}, \quad \forall \lambda \in \Lambda_{r_{1}, \theta}, \\
G(\lambda)=F(\lambda)(I-\widehat{B}(\lambda) F(\lambda))^{-1}, \quad \forall \lambda \in \Lambda_{r_{1}, \theta}, \\
\|A G(\lambda) x\| \leq \frac{M_{4}}{|\lambda|}\|x\|_{[D(A)]}, \quad \forall \lambda \in \Lambda_{r_{1}, \theta}, \quad x \in D(A), \\
\|A G(\lambda)\| \leq M_{5}, \quad \forall \lambda \in \Lambda_{r_{1}, \theta} .
\end{gathered}
$$

Demonstração: Sejam $\lambda \in \Lambda_{r, \theta}$ e $x \in X$. Do Lema 4.2 .1 vemos que

$$
\begin{aligned}
\|\widehat{B}(\lambda) F(\lambda) x\| & \leq \widehat{b}(\lambda)\left(\|F(\lambda) x\|_{[D(A)]}\right) \\
& \leq \widehat{b}(\lambda)(\|A F(\lambda) x\|+\|F(\lambda) x\|) \\
& \leq \widehat{b}(\lambda)\left(M_{2}+\frac{M_{1}}{|\lambda|}\right)\|x\| .
\end{aligned}
$$

Como $\lim \sup _{|\lambda| \rightarrow \infty} \widehat{b}(\lambda) M_{2}<1$, deduzimos a existência de $\delta \in(0,1)$ e de $r_{1}>r$ tais que $\widehat{b}(\lambda)\left(M_{2}+\frac{M_{1}}{\lambda \mid}\right) \leq \delta$ se $|\lambda|>r_{1}$. Isto implica que o operador $(I-\widehat{B}(\lambda) F(\lambda))$ é inversível e que $\left\|(I-\widehat{B}(\lambda) F(\lambda))^{-1}\right\| \leq \frac{1}{1-\delta}$ para $\lambda \in \Lambda_{r_{1}, \theta}$. 
Por outro lado, para $x \in X$ temos que

$$
\begin{aligned}
(\lambda+\lambda \widehat{N}(\lambda)-\widehat{B}(\lambda)-A) F(\lambda)(I-\widehat{B}(\lambda) F(\lambda))^{-1} x & = \\
(I-\widehat{B}(\lambda) F(\lambda))(\lambda+\lambda \widehat{N}(\lambda)-A) F(\lambda)(I-\widehat{B}(\lambda) F(\lambda))^{-1} x & = \\
(I-\widehat{B}(\lambda) F(\lambda))(I-\widehat{B}(\lambda) F(\lambda))^{-1} x & =x,
\end{aligned}
$$

e que quando $x \in D(A)$

$$
\begin{aligned}
F(\lambda)(I-\widehat{B}(\lambda) F(\lambda))^{-1}(\lambda+\lambda \widehat{N}(\lambda)-\widehat{B}(\lambda)-A) x & = \\
F(\lambda)(I-\widehat{B}(\lambda) F(\lambda))^{-1}(I-\widehat{B}(\lambda) F(\lambda))(\lambda+\lambda \widehat{N}(\lambda)-A) x & = \\
F(\lambda)(\lambda+\lambda \widehat{N}(\lambda)-A) x & =x .
\end{aligned}
$$

Portanto, o operador $(\lambda+\lambda \widehat{N}(\lambda)-\widehat{B}(\lambda)-A)$ possui inversa contínua para $\lambda \in \Lambda_{r_{1}, \theta}$, a qual é dada por $G(\lambda)=F(\lambda)(I-\widehat{B}(\lambda) F(\lambda))^{-1}$. Alêm disso, como $F(\lambda)(X) \subset D(A)$, temos que $G(\lambda)$ assume valores em $D(A)$ e que

$$
\begin{aligned}
\|G(\lambda)\| & \leq\|F(\lambda)\|\left\|(I-\widehat{B}(\lambda) F(\lambda))^{-1}\right\| \\
& \leq \frac{2 M_{1}}{|\lambda|} \frac{1}{1-\delta} \\
& \leq \frac{M_{3}}{|\lambda|} .
\end{aligned}
$$

Do anterior vemos que (4.9)-(4.10) são verificadas.

Vejamos agora que 4.11 e 4.12 também são válidas. Sejam $x \in D(A)$ e $\lambda \in \Lambda_{r_{1}, \theta}$. Observe que quando $\lambda \in \Lambda_{r_{1}, \theta}$ temos que $M_{2} \widehat{b}(\lambda)<1$ e $\widehat{b}(\lambda)\left(M_{2}+\frac{M_{1}}{|\lambda|}\right) \leq \delta$. Como conseqüência,

$$
\begin{aligned}
\|A G(\lambda) x\| & \leq\left\|A F(\lambda)(I-\widehat{B}(\lambda) F(\lambda))^{-1} x\right\| \\
& \leq\left\|A F(\lambda) \sum_{n=0}^{\infty}(\widehat{B}(\lambda) F(\lambda))^{n} x\right\| \\
& \leq\|A F(\lambda) x\|+\left\|A F(\lambda) \sum_{n=1}^{\infty}(\widehat{B}(\lambda) F(\lambda))^{n} x\right\| \\
& \leq\|A F(\lambda) x\|+\|A F(\lambda)\| \sum_{n=1}^{\infty}\left\|(\widehat{B}(\lambda) F(\lambda))^{n} x\right\| \\
& \leq \frac{M_{1}}{|\lambda|}\|x\|_{[D(A)]}+M_{2} \widehat{b}(\lambda) \sum_{n=1}^{\infty}\left(\left(M_{2}+\frac{M_{1}}{|\lambda|} \widehat{b}(\lambda)\right)^{n-1}\|F(\lambda) x\|_{[D(A)]}\right. \\
& \leq \frac{M_{1}}{|\lambda|}\|x\|_{[D(A)]}+\sum_{n=1}^{\infty} \delta^{n-1} \frac{2 M_{1}}{|\lambda|}\|x\|_{[D(A)]} \\
& \leq \frac{M_{4}}{|\lambda|}\|x\|_{[D(A)]} \cdot
\end{aligned}
$$


Finalmente, da definição de $G(\lambda)$ e 4.8 vemos que para $\lambda \in \Lambda_{r_{1}, \theta}$,

$$
\|A G(\lambda)\|=\left\|A F(\lambda)(I+\widehat{B}(\lambda) F(\lambda))^{-1}\right\| \leq M_{2} \frac{1}{1-\delta}=M_{5},
$$

o que permite concluir a demostração.

Observação 4.2.1 Antes de continuar é necessario enfatizar que a constante $r_{1}$ do Lema 4.2.2 foi escolhida de forma que $\widehat{b}(\lambda) M_{2}<1$ para todo $|\lambda|>r_{1}$ e que esta escolha é básica para a obtenção do resultado. Também observamos que o operador $\lambda \rightarrow G(\lambda)$ é escrito como o produto de operadores analíticos se $\lambda \in \Lambda_{r_{1}, \theta}$. Como as conseqüências do Lema 4.2.2 são de fundamental importância no que resta deste trabalho, achamos conveniente introduzir formalmente as seguintes hipóteses.

$\left(\mathbf{H N}_{\mathbf{5}}\right)$ Seja $M_{2}=2 M+2$ a constante garatida pelo Lema 4.2 .1 e $r_{0}>r_{1}$. Então $\widehat{b}(\lambda) M_{2}<1$ para todo $|\lambda| \geq r_{0}$.

$\left(\mathbf{H N}_{\mathbf{6}}\right)$ Os operadores $G(\lambda)$ e $A G(\lambda)$ são analíticos para todo $\lambda \in \Lambda_{r_{1}, \theta}$. Se $x \in D$, então $A G(\lambda) x=G(\lambda) A x$ para todo $\lambda \in \Lambda_{r_{1}, \theta}$.

No que segue, usaremos a técnica de transformada de Laplace para definir uma família N-resolvente $(R(t))_{t \geq 0}$ para 4.1)-(4.2). Também mostraremos que $(R(t))_{t \geq 0}$ possui uma extensão analítica a uma região apropriada do plano complexo.

No que segue deste capítulo, assumiremos que as condições $\left(\mathbf{H N}_{\mathbf{1}}\right)-\left(\mathbf{H N}_{\mathbf{6}}\right)$ são verificadas e que $M_{1}, \cdots, M_{5}$ são as constantes dos Lemas 4.2.1, 4.2.2. No resto da seção, $\phi$ será um número fixo em $\left(\frac{\pi}{2}, \theta\right)$ e $r_{0}>r_{1}$. Para $r>0, \Gamma(r)=\Gamma_{r}^{1} \cup \Gamma_{r}^{2} \cup \Gamma_{r}^{3}$ será a curva dada por

$$
\begin{aligned}
\Gamma_{r}^{1} & =\left\{\gamma e^{i \phi}: \gamma \geq r\right\} \\
\Gamma_{r}^{2} & =\left\{r e^{i \xi}:-\phi \leq \xi \leq \phi\right\} \\
\Gamma_{r}^{3} & =\left\{-\gamma e^{-i \phi}: \gamma \leq-r\right\} .
\end{aligned}
$$

onde cada curva $\Gamma_{r}^{i}, i=1,2,3$ é orientada de forma que $\operatorname{Im}(\lambda)$ é crescente em $\Gamma_{r}^{1}$ e $\Gamma_{r}^{3}$.

Usando as notações anteriores, definimos a família de operadores $(R(t))_{t \geq 0}$ por

$$
R(t)=\left\{\begin{array}{cc}
\frac{1}{2 \pi i} \int_{\Gamma\left(r_{0}\right)} e^{\lambda t}(\lambda+\lambda \widehat{N}(\lambda)-\widehat{B}(\lambda)-A)^{-1} d \lambda, & t>0, \\
I d, & t=0 .
\end{array}\right.
$$

Nos próximos Lemas, mostraremos que $(R(t))_{t \geq 0}$ é uma família N-resolvente para 4.1)(4.2). 
Lema 4.2.3 Suponha que $\left(\mathbf{H N}_{\mathbf{1}}\right)-\left(\mathbf{H N}_{\mathbf{6}}\right)$ são satisfeitas. Então, $R(t) \in \mathcal{L}(X)$ para todo $t \geq 0$.

Demonstração: Se $x \in X$, do Lema 4.2 .2 segue que

$$
\begin{aligned}
\left\|\frac{1}{2 \pi i} \int_{\Gamma\left(r_{0}\right)} e^{\lambda t} G(\lambda) x d \lambda\right\| & \leq \frac{1}{\pi} \int_{r_{0}}^{\infty}\left|e^{\lambda t}\right| \frac{M_{3}}{|\lambda|}|d \lambda|\|x\|+\frac{1}{2 \pi} \int_{-\phi}^{\phi}\left|e^{\lambda t}\right| \frac{M_{3}}{|\lambda|}|d \lambda|\|x\| \\
& \leq \frac{1}{\pi} \int_{r_{0}}^{\infty} e^{-r t \operatorname{sen}\left(\phi-\frac{\pi}{2}\right)} \frac{M_{3}}{r} d r\|x\|+\frac{1}{2 \pi} \int_{-\phi}^{\phi} e^{r_{0} t \cos \xi} \frac{M_{3}}{r_{0}} r_{0} d \xi\|x\| \\
& \leq\left(\frac{M_{3}}{\pi r_{0}} \frac{e^{-r_{0} \operatorname{tsen}\left(\phi-\frac{\pi}{2}\right)}}{t \operatorname{sen}\left(\phi-\frac{\pi}{2}\right)}+\frac{M_{3}}{\pi} e^{r_{0} t} \phi\right)\|x\|,
\end{aligned}
$$

o que prova que $R(t) \in \mathcal{L}(X)$ para todo $t \geq 0$, pois $R(0)=I$.

Lema 4.2.4 Se $\left(\mathbf{H N}_{\mathbf{1}}\right)-\left(\mathbf{H N}_{\mathbf{6}}\right)$ são satisfeitas, então $(R(t))_{t \geq 0}$ é família uniformemente limitada em $\mathcal{L}(X)$.

Demonstração: Estudaremos inicialmente a limitação de $R(t)$ para $t \geq 1$. Sejam $x \in X$ e $t \geq 1$. Fazendo a mudança de coordenada $\lambda t=\gamma$, é fácil ver que

$$
R(t) x=\frac{1}{2 \pi i} \int_{\Gamma\left(t r_{0}\right)} t^{-1} e^{\gamma} G\left(\gamma t^{-1}\right) x d \gamma .
$$

Mais ainda, como $G(\cdot)$ é analítica em $\Lambda_{r_{1}, \theta}$, do Teorema de Cauchy podemos concluir que

$$
R(t) x=\frac{1}{2 \pi i} \int_{\Gamma\left(r_{0}\right)} t^{-1} e^{\gamma} G\left(\gamma t^{-1}\right) x d \gamma
$$

Nestas condições, do Lema 4.2 .2 obtemos que

$$
\begin{aligned}
\|R(t) x\| & \leq \frac{1}{\pi} \int_{r_{0}}^{\infty}\left|e^{\gamma}\right| \frac{M_{3}}{|\gamma|}|d \gamma|\|x\|+\frac{1}{2 \pi} \int_{-\phi}^{\phi}\left|e^{\gamma}\right| \frac{M_{3}}{|\gamma|}|d \gamma|\|x\| \\
& \leq \frac{1}{\pi} \int_{r_{0}}^{\infty} e^{-r \operatorname{sen}\left(\phi-\frac{\pi}{2}\right)} \frac{M_{3}}{r} d r\|x\|+\frac{1}{2 \pi} \int_{-\phi}^{\phi} e^{r_{0} \cos \xi} \frac{M_{3}}{r_{0}} r_{0} d \xi\|x\| \\
& \leq\left(\frac{M_{3}}{\pi r_{0}} \frac{e^{-r_{0} \operatorname{sen}\left(\phi-\frac{\pi}{2}\right)}}{\operatorname{sen}\left(\phi-\frac{\pi}{2}\right)}+\frac{M_{3}}{\pi} e^{r_{0}} \phi\right)\|x\| .
\end{aligned}
$$

O anterior mostra que $\{R(t): t \geq 1\}$ é limitado em $\mathcal{L}(X)$.

Estudemos agora o caso onde $t \in(0,1)$. Como $t<1$, segue que $t^{-1} r_{0}>r_{0}$. Usando como antes a analiticidade de $G(\cdot)$ e o Teorema de Cauchy, segue que

$$
R(t) x=\frac{1}{2 \pi i} \int_{\Gamma\left(t^{-1} r_{0}\right)} e^{\lambda t} G(\lambda) x d \lambda .
$$


Como consequência,

$$
\begin{aligned}
\|R(t) x\| & \leq \frac{1}{\pi} \int_{t^{-1} r_{0}}^{\infty}\left|e^{\lambda t}\right| \frac{M_{3}}{|\lambda|}|d \lambda|\|x\|+\frac{1}{2 \pi} \int_{-\phi}^{\phi}\left|e^{\lambda t}\right| \frac{M_{3}}{|\lambda|}|d \lambda|\|x\| \\
& \leq \frac{1}{\pi} \int_{t^{-1} r_{0}}^{\infty} e^{-r t \operatorname{sen}\left(\phi-\frac{\pi}{2}\right)} \frac{M_{3}}{r} d r\|x\|+\frac{1}{2 \pi} \int_{-\phi}^{\phi} e^{t^{-1} r_{0} t \cos \xi} \frac{M_{3}}{t^{-1} r_{0}} t^{-1} r_{0} d \xi\|x\| \\
& \leq\left(\frac{M_{3}}{\pi r_{0}} \frac{e^{-r_{0} \operatorname{sen}\left(\phi-\frac{\pi}{2}\right)}}{\operatorname{sen}\left(\phi-\frac{\pi}{2}\right)}+\frac{M_{3}}{\pi} e^{r_{0}} \phi\right)\|x\|,
\end{aligned}
$$

o que prova que $\{R(t): t \in(0,1)\}$ é limitado em $\mathcal{L}(X)$. Agora a demostração está completa.

Lema 4.2.5 Se $\left(\mathbf{H N}_{\mathbf{1}}\right)-\left(\mathbf{H N}_{\mathbf{6}}\right)$ são válidas, então $R(\cdot) x \in C([0, \infty): X)$ para todo $x \in X$.

Demonstração: Sejam $t>0, x \in X$. Observe que para $r_{t}>r_{0}$ e $s>0$ temos que

$$
\begin{aligned}
\left\|\int_{\Gamma\left(r_{0}\right) \cap\left\{\lambda:|\lambda| \geq r_{t}\right\}} e^{\lambda s} G(\lambda) x d \lambda\right\| & \leq \frac{1}{\pi} \int_{r_{t}}^{\infty}\left|e^{\lambda s}\right| \frac{M_{3}}{|\lambda|}|d \lambda|\|x\| \\
& \leq \frac{1}{\pi} \int_{r_{t}}^{\infty} e^{-r s \operatorname{sen}\left(\phi-\frac{\pi}{2}\right)} \frac{M_{3}}{r} d r\|x\| \\
& \leq \frac{M_{3}}{\pi r_{t}} \frac{e^{-r_{t} s \operatorname{sen}\left(\phi-\frac{\pi}{2}\right)}}{s \operatorname{sen}\left(\phi-\frac{\pi}{2}\right)}\|x\| .
\end{aligned}
$$

Portanto, dado $\epsilon>0$, podemos escolher $r_{t}>r_{0}$ tal que

$$
\left\|\int_{\Gamma\left(r_{0}\right) \cap\left\{\lambda:|\lambda| \geq r_{t}\right\}} e^{\lambda s} G(\lambda) d \lambda\right\|<\epsilon,
$$

para todo $s \in\left[\frac{t}{2}, \frac{3 t}{2}\right]$. Por outro lado, como $e^{\lambda s} G(\lambda) \rightarrow e^{\lambda t} G(\lambda)$ quando $s \rightarrow t$, uniformemente sobre $\Gamma\left(r_{0}\right) \cap\left\{\lambda:|\lambda| \leq r_{t}\right\}$, existe $\delta_{t}>0$ tal que

$$
\left\|\int_{\Gamma\left(r_{0}\right) \cap\left\{\lambda:|\lambda| \leq r_{t}\right\}} e^{\lambda s} G(\lambda) d \lambda-\int_{\Gamma\left(r_{0}\right) \cap\left\{\lambda:|\lambda| \leq r_{t}\right\}} e^{\lambda t} G(\lambda) d \lambda\right\|<\epsilon
$$

se $|t-s|<\delta_{t}$. De 4.14 e 4.15) concluímos que $\|R(t)-R(s)\|_{\mathcal{L}(X)}<2 \epsilon$ se $|t-s|<\delta_{t}$, o que em particular prova que $R(\cdot) x$ é contínua se $t>0$.

Mostraremos agora que $R(\cdot) x$ é contínua em zero. Estudemos inicialmente o caso onde $x \in D(A)$. Se $\lambda \in \Lambda_{r_{1}, \theta}$, temos que $G(\lambda)(\lambda+\lambda \widehat{N}(\lambda)-\widehat{B}(\lambda)-A) x=x$, e então

$$
G(\lambda) x-\lambda^{-1} x=\lambda^{-1} G(\lambda)(\lambda \widehat{N}(\lambda)-\widehat{B}(\lambda)-A) x
$$

e

$$
\begin{aligned}
R(t) x-x & =\frac{1}{2 \pi i} \int_{\Gamma\left(r_{0}\right)} e^{\lambda t} G(\lambda) x d \lambda-x \\
& =\frac{1}{2 \pi i} \int_{\Gamma\left(r_{0}\right)}\left(e^{\lambda t} G(\lambda) x-\lambda^{-1} e^{\lambda t} x\right) d \lambda \\
& =\frac{1}{2 \pi i} \int_{\Gamma\left(r_{0}\right)} e^{\lambda t} \lambda^{-1} G(\lambda)(\lambda \widehat{N}(\lambda)-\widehat{B}(\lambda)-A) x d \lambda
\end{aligned}
$$


Pelas hipóteses $\left(\mathbf{H N}_{\mathbf{1}}\right)-\left(\mathbf{H N}_{\mathbf{3}}\right)$ e o Lema 4.2 .2 temos que $\left\|e^{\lambda t} \lambda^{-1} G(\lambda)(\lambda \widehat{N}(\lambda)-\widehat{B}(\lambda)-A) x\right\| \leq M_{3}\left|e^{\lambda t}\right|\left(\frac{N}{|\lambda|^{1+\alpha}}+\frac{\widehat{b}(\lambda)}{|\lambda|^{2}}+\frac{1}{|\lambda|^{2}}\right)\|x\|_{[D(A)]}$, e o lado direito da desigualdade acima é integrável independentemente de $t$, por uma simples aplicação do Teorema da Convergência dominada de Lebesgue podemos concluir que

$$
\lim _{t \rightarrow 0^{+}}(R(t) x-x)=\frac{1}{2 \pi i} \int_{\Gamma\left(r_{0}\right)} \lambda^{-1} G(\lambda)(\lambda \widehat{N}(\lambda)-\widehat{B}(\lambda)-A) x d \lambda .
$$

Mostraremos agora que o termo a direita da última desigualdade é zero. Para isto, considere os caminhos $C^{n}=\left\{n e^{i \xi}: \phi \leq \xi \leq-\phi\right\}$ e $\Gamma\left(r_{0}\right) \bigcap\{\lambda ;|\lambda| \leq n\} \cup C^{n}$ orientados de maneira de preservar a orientação em $\Gamma\left(r_{0}\right)$. Do Teorema de Cauchy, para cada $n>r_{0}$, temos que

$$
\frac{1}{2 \pi i} \int_{\Gamma\left(r_{0}\right) \bigcap\{\lambda ;|\lambda| \leq n\} \cup C^{n}} \lambda^{-1} G(\lambda)(\lambda \widehat{N}(\lambda)-\widehat{B}(\lambda)-A) x d \lambda=0 .
$$

Por outro lado, das hipóteses $\left(\mathbf{H N}_{\mathbf{1}}\right)-\left(\mathbf{H N}_{\mathbf{3}}\right)$ e do Lema 4.2 .2 obtemos a estimativa

$$
\begin{aligned}
& \left\|\frac{1}{2 \pi i} \int_{C^{n}} \lambda^{-1} G(\lambda)(\lambda \widehat{N}(\lambda)-\widehat{B}(\lambda)-A) x d \lambda\right\| \\
& \quad \leq \frac{1}{2 \pi} \int_{-\phi}^{\phi} \frac{M_{3} N}{n^{1+\alpha}} n d \xi\|x\|+\frac{1}{2 \pi} \int_{-\phi}^{\phi} \frac{M_{3}}{M_{2} n^{2}} n d \xi\|x\|_{[D(A)]}+\frac{1}{2 \pi} \int_{-\phi}^{\phi} \frac{M_{3}}{n^{2}} n d \xi\|x\|_{[D(A)]},
\end{aligned}
$$

de onde segue que

$$
\frac{1}{2 \pi i} \int_{C^{n}} \lambda^{-1} G(\lambda)(\lambda \widehat{N}(\lambda)-\widehat{B}(\lambda)-A) x d \lambda \rightarrow 0
$$

quando $n \rightarrow \infty$. Assim vemos que

$$
\begin{aligned}
& \frac{1}{2 \pi i} \int_{\Gamma\left(r_{0}\right)} \lambda^{-1} G(\lambda)(\lambda \widehat{N}(\lambda)-\widehat{B}(\lambda)-A) x d \lambda \\
&=\lim _{n \rightarrow \infty} \frac{1}{2 \pi i} \int_{\Gamma\left(r_{0}\right) \cap\{\lambda ;|\lambda| \leq n\}} \lambda^{-1} G(\lambda)(\lambda \widehat{N}(\lambda)-\widehat{B}(\lambda)-A) x d \lambda \\
&+\lim _{n \rightarrow \infty} \frac{1}{2 \pi i} \int_{C^{n}} \lambda^{-1} G(\lambda)(\lambda \widehat{N}(\lambda)-\widehat{B}(\lambda)-A) x d \lambda \\
&= \lim _{n \rightarrow \infty} \frac{1}{2 \pi i} \int_{\Gamma\left(r_{0}\right) \bigcap\{\lambda ;|\lambda| \leq n\} \cup C^{n}} \lambda^{-1} G(\lambda)(\lambda \widehat{N}(\lambda)-\widehat{B}(\lambda)-A) x d \lambda=0,
\end{aligned}
$$

o que prova que $R(t) x \rightarrow x$ se $t \rightarrow 0^{+}$para todo $x \in D(A)$.

Finalmente, do Lema 4.2 .4 sabemos que $(R(t))_{t \geq 0}$ é uniformemente limitada sobre $[0, \infty)$, o que junto a densidade de $D(A)$ em $X$ permite concluir que $R(\cdot) x$ é contínua em zero para todo $x \in X$.

Nos próximos Lemas estudamos a diferenciabilidade de $R(\cdot) x$. 
Lema 4.2.6 Se as condições $\left(\mathbf{H N}_{\mathbf{1}}\right)-\left(\mathbf{H N}_{\mathbf{6}}\right)$ são válidas, então a função $R(\cdot) x \in C^{1}((0, \infty)$ : $X)$ para todo $x \in X e$

$$
R^{\prime}(t) x=\frac{1}{2 \pi i} \int_{\Gamma} \lambda e^{\lambda t} G(\lambda) x d \lambda .
$$

Demonstração: Para $x \in X, h>0$ e $t>0$,

$$
\frac{R(t+h) x-R(t) x}{h}=\frac{1}{2 \pi i} \int_{\Gamma} \frac{e^{\lambda h}-1}{h} e^{\lambda t} G(\lambda) x d \lambda .
$$

É fácil mostrar que $\lambda e^{\lambda t}$ é integrável sobre $\Gamma\left(r_{0}\right)$. Este fato, junto a estimativa

$$
\left\|\frac{e^{\lambda h}-1}{h} e^{\lambda t} G(\lambda)\right\| \leq|\lambda| e\left|e^{\lambda t}\right| \frac{M_{3}}{|\lambda|},
$$

permite concluir por uma aplicação do Teorema da convêrgencia dominada que $R(\cdot) x$ é diferenciável em $t$ e que

$$
R^{\prime}(t) x=\frac{1}{2 \pi i} \int_{\Gamma} \lambda e^{\lambda t} G(\lambda) x d \lambda
$$

Usando a estimativa

$$
\left\|\lambda e^{\lambda s} G(\lambda)\right\| \leq\left|\lambda \| e^{\lambda s}\right| \frac{M_{3}}{|\lambda|}
$$

e procedendo como na primeira parte da prova do Lema 4.2.5, podemos mostrar a continuidade de $R^{\prime}(\cdot)$ sobre $(0, \infty)$, em particular a continuidade de $R^{\prime}(\cdot) x$ sobre $(0, \infty)$. Para não estender o texto, omitiremos esta parte da prova. A demostração está completa.

As provas dos seguintes Lemas são similares as provas dos Lemas 4.2.4, 4.2.5. Por isto, serão resumidas convenientemente. Como sempre, assumiremos nos próximos resultados que $\left(\mathbf{H N}_{\mathbf{1}}\right)-\left(\mathbf{H N}_{\mathbf{6}}\right)$ são satisfeitas.

Lema 4.2.7 A família $(R(t))_{t \geq 0}$ é uniformemente limitada em $\mathcal{L}([D(A)])$.

Demonstração: Para $x \in D(A)$ e $t>0$ definamos o operador $(S(t))_{t>0}$ por

$$
S(t) x=\frac{1}{2 \pi i} \int_{\Gamma} e^{\lambda t} A G(\lambda) x d \lambda .
$$

Para $t \geq 1$ considere a mudança de coordenada $\lambda t=\gamma$. É fácil ver que

$$
S(t) x=\frac{1}{2 \pi i} \int_{\Gamma\left(t r_{0}\right)} t^{-1} e^{\gamma} A G\left(\gamma t^{-1}\right) x d \gamma .
$$

Como $A G(\cdot)$ é analítica em $\Lambda_{r_{1}, \theta}$, pelo Teorema de Cauchy podemos concluir que

$$
S(t) x=\frac{1}{2 \pi i} \int_{\Gamma\left(r_{0}\right)} t^{-1} e^{\gamma} A G\left(\gamma t^{-1}\right) x d \gamma .
$$


Nestas condições, do Lema 4.2 .2 obtemos que

$$
\begin{aligned}
\|S(t) x\| & \leq \frac{1}{\pi} \int_{r_{0}}^{\infty}\left|e^{\gamma}\right| \frac{M_{4}}{|\gamma|}|d \gamma|\|x\|_{[D(A)]}+\frac{1}{2 \pi} \int_{-\phi}^{\phi}\left|e^{\gamma}\right| \frac{M_{4}}{|\gamma|}|d \gamma|\|x\|_{[D(A)]} \\
& \leq \frac{1}{\pi} \int_{r_{0}}^{\infty} e^{-r \operatorname{sen}\left(\phi-\frac{\pi}{2}\right)} \frac{M_{4}}{r} d r\|x\|_{[D(A)]}+\frac{1}{2 \pi} \int_{-\phi}^{\phi} e^{r_{0} \cos \xi} \frac{M_{4}}{r_{0}} r_{0} d \xi\|x\|_{[D(A)]} \\
& \leq\left(\frac{M_{4}}{\pi r_{0}} \frac{e^{-r_{0} \operatorname{sen}\left(\phi-\frac{\pi}{2}\right)}}{\operatorname{sen}\left(\phi-\frac{\pi}{2}\right)}+\frac{M_{4}}{\pi} e^{r_{0}} \phi\right)\|x\|_{[D(A)]} .
\end{aligned}
$$

Segue do anterior temos que $\{S(t): t \geq 1\}$ é limitado em $\mathcal{L}([D(A)]: X)$.

Estudemos agora o caso onde $t \in(0,1)$. Observe que $t^{-1} r_{0}>r_{0}$. Usando como antes a analiticidade de $A G(\cdot)$ e o Teorema de Cauchy segue que

$$
S(t) x=\frac{1}{2 \pi i} \int_{\Gamma\left(t^{-1} r_{0}\right)} e^{\lambda t} G(\lambda) x d \lambda .
$$

Como consequência,

$$
\begin{aligned}
\| & S(t) x \| \\
& \leq \frac{1}{\pi} \int_{t^{-1} r_{0}}^{\infty}\left|e^{\lambda t}\right| \frac{M_{4}}{|\lambda|}|d \lambda|\|x\|_{[D(A)]}+\frac{1}{2 \pi} \int_{-\phi}^{\phi}\left|e^{\lambda t}\right| \frac{M_{4}}{|\lambda|}|d \lambda|\|x\|_{[D(A)]} \\
& \leq \frac{1}{\pi} \int_{t^{-1} r_{0}}^{\infty} e^{-r t \operatorname{sen}\left(\phi-\frac{\pi}{2}\right)} \frac{M_{4}}{r} d r\|x\|_{[D(A)]}+\frac{1}{2 \pi} \int_{-\phi}^{\phi} e^{t^{-1} r_{0} t \cos \xi} \frac{M_{4}}{t^{-1} r_{0}} t^{-1} r_{0} d \xi\|x\|_{[D(A)]} \\
& \leq\left(\frac{M_{4}}{\pi r_{0}} \frac{e^{-r_{0} \operatorname{sen}\left(\phi-\frac{\pi}{2}\right)}}{\operatorname{sen}\left(\phi-\frac{\pi}{2}\right)}+\frac{M_{4}}{\pi} e^{r_{0}} \phi\right)\|x\|_{[D(A)]},
\end{aligned}
$$

para $t>0$ e $x \in D(A)$. Isto, mostra que a família de operadores $(S(t))_{t>0}$ é uniformemente limitada em $\mathcal{L}([D(A)]: X)$. Usando agora que $A$ é fechado, obtemos que $A R(t)=S(t)$ e assim que $(R(t))_{t \geq 0}$ é uniformemente limitada em $\mathcal{L}([D(A)])$. A prova está agora completa.

Lema 4.2.8 $R(\cdot) x \in C([0, \infty):[D(A)])$ para todo $x \in D(A)$.

Demonstração: Procedendo como no Lema 4.2 .5 podemos mostrar que $A R(\cdot) x$ é contínua sobre $(0, \infty)$ para todo $x \in D(A)$.

Para analisar a continuidade no zero, estudaremos inicialmente o caso onde $x \in D$, sendo $D$ o conjunto introduzido em $\left(\mathbf{H N}_{\mathbf{4}}\right)$. Neste caso,

$$
G(\lambda) x-\lambda^{-1} x=\lambda^{-1} G(\lambda)(\lambda \widehat{N}(\lambda)-\widehat{B}(\lambda)-A) x,
$$

o que do Lema 4.2 .2 e $\left(\mathbf{H N}_{\mathbf{6}}\right)$ implica que

$$
\begin{aligned}
& \left\|e^{\lambda t} \lambda^{-1} A G(\lambda)(\lambda \widehat{N}(\lambda)-\widehat{B}(\lambda)-A) x\right\| \\
& \quad \leq \quad M_{3}\left|e^{\lambda t}\right|\left(\frac{N}{\left.|\lambda|\right|^{1+\alpha}}\|x\|_{[D(A)]}+\frac{\widehat{b}(\lambda)+1}{|\lambda|^{2}}\left(\|x\|_{[D(A)]}+\|A x\|_{[D(A)]}\right)\right) .
\end{aligned}
$$


Agora, como consequêcia de que a função do lado direito em (4.17) é integrável sobre $\Gamma\left(r_{0}\right)$ independentemente de $t$, segue do Teorema da convergência dominada que

$$
\begin{aligned}
\lim _{t \rightarrow 0^{+}}(A R(t) x-A x) & =\lim _{t \rightarrow 0^{+}} \frac{1}{2 \pi i} \int_{\Gamma\left(r_{0}\right)} e^{\lambda t}\left(A G(\lambda) x-\lambda^{-1} A x\right) d \lambda \\
& =\lim _{t \rightarrow 0^{+}} \frac{1}{2 \pi i} \int_{\Gamma\left(r_{0}\right)} e^{\lambda t} \lambda^{-1} A G(\lambda)(\lambda \widehat{N}(\lambda)-\widehat{B}(\lambda)-A) x d \lambda \\
& =\frac{1}{2 \pi i} \int_{\Gamma\left(r_{0}\right)} \lambda^{-1} A G(\lambda)(\lambda \widehat{N}(\lambda)-\widehat{B}(\lambda)-A) x d \lambda .
\end{aligned}
$$

Assim, para mostrar que a propriedade é válida para $x \in D$, é suficiente provar que

$$
\frac{1}{2 \pi i} \int_{\Gamma\left(r_{0}\right)} \lambda^{-1} A G(\lambda)(\lambda \widehat{N}(\lambda)-\widehat{B}(\lambda)-A) x d \lambda=0 .
$$

Para mostrar isto, vamos proceder como na prova do Lema 4.2.5. Sejam os caminhos $C^{n}=$ $\left\{n e^{i \xi}:-\phi \leq \xi \leq \phi\right\}$ e $\Gamma\left(r_{0}\right) \bigcap\{\lambda ;|\lambda| \leq n\} \cup C^{n}$ orientados de forma de preservar a orientação original em $\Gamma\left(r_{0}\right)$. Observamos que da desigualdade (4.17) segue facilmente que

$$
\lim _{n \rightarrow \infty} \frac{1}{2 \pi i} \int_{C^{n}} \lambda^{-1} G(\lambda)(\lambda \widehat{N}(\lambda)-\widehat{B}(\lambda)-A) x d \lambda=0
$$

Agora, da analiticidade de $\lambda^{-1} G(\lambda)(\lambda \widehat{N}(\lambda)-\widehat{B}(\lambda)-A) x$ sobre $\Lambda_{r_{1}, \theta}$ e o Teorema de Cauchy, vemos que

$$
\begin{aligned}
0= & \lim _{n \rightarrow \infty} \frac{1}{2 \pi i} \int_{\Gamma\left(r_{0}\right) \cap\{\lambda ;|\lambda| \leq n\} \cup C^{n}} \lambda^{-1} A G(\lambda)(\lambda \widehat{N}(\lambda)-\widehat{B}(\lambda)-A) x d \lambda \\
= & \lim _{n \rightarrow \infty} \frac{1}{2 \pi i} \int_{\Gamma\left(r_{0}\right) \cap\{\lambda ;|\lambda| \leq n\}} \lambda^{-1} A G(\lambda)(\lambda \widehat{N}(\lambda)-\widehat{B}(\lambda)-A) x d \lambda \\
& +\lim _{n \rightarrow \infty} \frac{1}{2 \pi i} \int_{C^{n}} \lambda^{-1} A G(\lambda)(\lambda \widehat{N}(\lambda)-\widehat{B}(\lambda)-A) x d \lambda \\
= & \frac{1}{2 \pi i} \int_{\Gamma} \lambda^{-1} A G(\lambda)(\lambda \widehat{N}(\lambda)-\widehat{B}(\lambda)-A) x d \lambda,
\end{aligned}
$$

o que mostra que $\lim _{t \downarrow 0} A R(t) x=A x$ para todo $x \in D$.

Finalmente, como $D$ é denso em $[D(A)]$ e $(R(t))_{t \geq 0}$ é uniformemente limitada em $\mathcal{L}([D(A)])$, concluímos que a propriedade é válida para todo $x \in D(A)$. A prova está completa.

Lema 4.2.9 $\widehat{R}(\lambda) x=G(\lambda) x$ para todo $\lambda \in \Lambda_{r_{1}, \theta}$ e todo $x \in X$.

Demonstração: Da definição de $(R(t))_{t \geq 0}$, vemos que

$$
\begin{aligned}
\widehat{R}(\lambda) x & =\frac{1}{2 \pi i} \int_{0}^{\infty} e^{-\lambda t} \int_{\Gamma\left(r_{0}\right)} e^{\gamma t} G(\gamma) x d \gamma d t \\
& =\frac{1}{2 \pi i} \int_{\Gamma\left(r_{0}\right)} \int_{0}^{\infty} e^{-(\lambda-\gamma) t} G(\gamma) x d t d \gamma \\
& =\frac{1}{2 \pi i} \int_{\Gamma\left(r_{0}\right)}(\lambda-\gamma)^{-1} G(\gamma) x d \gamma
\end{aligned}
$$


Se as curvas $C^{n}=\left\{n e^{i \xi}: \phi \leq \xi \leq \phi\right\}$ e $\Gamma\left(r_{0}\right) \bigcap\{\lambda ;|\lambda| \leq n\} \cup C^{n}, n>r_{0}$, são orientadas de forma de manter a orientação em $\Gamma\left(r_{0}\right)$, da Teoria clássica de funções analíticas obtemos que

$$
G(\lambda) x=\frac{1}{2 \pi i} \int_{\Gamma\left(r_{0}\right) \bigcap\{\lambda ;|\lambda| \leq n\} \cup C^{n}}(\lambda-\gamma)^{-1} G(\gamma) x d \gamma, \quad \lambda \in \Lambda_{r_{1}, \theta},|\lambda|<n .
$$

Por outro lado, da estimativa

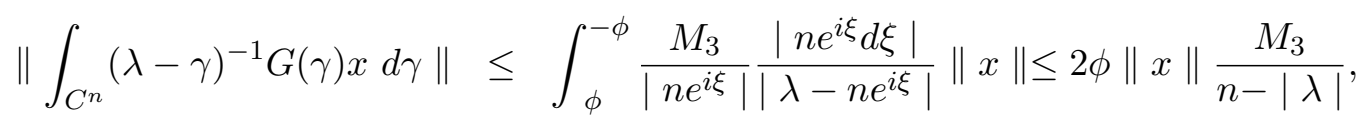

concluímos que $\frac{1}{2 \pi i} \int_{C^{n}}(\lambda-\gamma)^{-1} G(\gamma) x d \gamma \rightarrow 0$ quando $n \rightarrow \infty$. Das observações anteriores, segue que para $\lambda \in \Lambda_{r_{1}, \theta}$

$$
\begin{aligned}
\widehat{R}(\lambda) x=\frac{1}{2 \pi i} \int_{\Gamma\left(r_{0}\right)}(\lambda-\gamma)^{-1} G(\gamma) x d \gamma= & \lim _{n \rightarrow \infty} \frac{1}{2 \pi i} \int_{\Gamma\left(r_{0}\right) \bigcap\{\lambda ;|\lambda| \leq n\}}(\lambda-\gamma)^{-1} G(\gamma) x d \gamma \\
& +\lim _{n \rightarrow \infty} \frac{1}{2 \pi i} \int_{C^{n}}(\lambda-\gamma)^{-1} G(\gamma) x d \gamma \\
= & G(\lambda) x .
\end{aligned}
$$

Portanto, $\widehat{R}(\lambda) x=G(\lambda) x$ para todo $\lambda \in \Lambda_{r_{1}, \theta}$ e todo $x \in X$.

Lema 4.2.10 A família $(R(t))_{t \geq 0}$ satisfaz as equações (4.3)-(4.4). Mais ainda, se $x \in D(A)$, então $R(\cdot) x+(R * N)(\cdot) x \in C^{1}([0, \infty): X)$ e $R(\cdot) x+(N * R)(\cdot) x \in C^{1}([0, \infty): X)$.

Demonstração: Sejam $x \in D(A)$ e $M>0$ tal que $\|R(t)\| \leq M$ para todo $t \geq 0$. Mostraremos inicialmente que a função $R(\cdot)$ verifica (4.4) para $t>0$. Usando que a integral $\frac{1}{2 \pi i} \int_{\Gamma\left(r_{0}\right)} e^{\lambda t} x d \lambda=0$, dos Lemas 4.2.5 4.2.6 e 4.2.9. e do fato que as funções $\lambda e^{\lambda} G(\lambda) \widehat{N}(\lambda) x$ e $\lambda e^{\lambda} G(\lambda) \widehat{B}(\lambda) x$ são integráveis sobre $\Gamma\left(r_{0}\right)$, segue que

$$
\begin{aligned}
R^{\prime}(t) x & =\frac{1}{2 \pi i} \int_{\Gamma\left(r_{0}\right)} \lambda e^{\lambda t} G(\lambda) x d \lambda \\
& =\frac{1}{2 \pi i} \int_{\Gamma\left(r_{0}\right)} e^{\lambda t}[x-G(\lambda)(\lambda \widehat{N}(\lambda)-\widehat{B}(\lambda)-A) x] d \lambda \\
& =R(t) A x-\frac{1}{2 \pi i} \int_{\Gamma\left(r_{0}\right)} \lambda e^{\lambda t} \widehat{R}(\lambda) \widehat{N}(\lambda) x d \lambda+\frac{1}{2 \pi i} \int_{\Gamma\left(r_{0}\right)} e^{\lambda t} \widehat{R}(\lambda) \widehat{B}(\lambda) x d \lambda
\end{aligned}
$$

Assim, para mostrar que se verifica (4.4), é suficiente provar que

$$
\begin{aligned}
\frac{1}{2 \pi i} \int_{\Gamma\left(r_{0}\right)} \lambda e^{\lambda t} \widehat{R}(\lambda) \widehat{N}(\lambda) x d \lambda & =\frac{d}{d t} \int_{0}^{t} R(t-s) N(s) x d s, \\
\frac{1}{2 \pi i} \int_{\Gamma\left(r_{0}\right)} e^{\lambda t} \widehat{R}(\lambda) \widehat{B}(\lambda) x d \lambda & =\int_{0}^{t} R(t-s) B(s) x d s .
\end{aligned}
$$


Estudemos em primeiro lugar o termo (4.18). Definamos a função $\Theta:[0, \infty) \rightarrow X$ mediante a expresão $\Theta(s)=\int_{0}^{s} R(s-\mu) N(\mu) x d \mu$. Como $N(\cdot) x \in L^{1}\left(\mathbb{R}^{+}: X\right)$, é claro que $\Theta \in C([0, \infty): X)$. Mais ainda, $\Theta$ é limitada sobre $[0, \infty)$ pois $\|\Theta(s)\| \leq M\|N\|_{L^{1}\left(\mathbb{R}^{+}: \mathcal{L}(X)\right)}$ para todo $s \geq 0$. Seja agora $\xi(s)=\int_{0}^{s} \Theta(\mu) d \mu$. Nestas condições, a Transformada de Laplace-Stieltjes de $\xi$ existe para $\operatorname{Re}(\lambda)>0$ e

$$
\int_{0}^{\infty} e^{-\lambda t} d \xi(t)=\int_{0}^{\infty} e^{-\lambda t} \Theta(t) d t=\widehat{R}(\lambda) \widehat{N}(\lambda) x
$$

Agora, pelo Teorema da Transformada de Laplace inversa, veja [48, Theorem 6.3.1], e da definição de $\xi(\cdot)$, vemos que para $\gamma>r_{0}$

$$
\xi(t)=\frac{1}{2 \pi i} \int_{\gamma-i \infty}^{\gamma+i \infty} e^{\lambda t} \int_{0}^{\infty} e^{-\lambda t} d \xi(t) \frac{d \lambda}{\lambda}=\frac{1}{2 \pi i} \int_{\gamma-i \infty}^{\gamma+i \infty} e^{\lambda t} \widehat{R}(\lambda) \widehat{N}(\lambda) x \frac{d \lambda}{\lambda}
$$

de onde obtemos que

$$
\frac{d}{d t} \Theta(t)=\frac{1}{2 \pi i} \int_{\gamma-i \infty}^{\gamma+i \infty} \lambda e^{\lambda t} \widehat{R}(\lambda) \widehat{N}(\lambda) x d \lambda
$$

Consequêntemente, para finalizar esta parte da demonstração é suficiente provar

$$
\frac{1}{2 \pi i} \int_{\gamma-i \infty}^{\gamma+i \infty} \lambda e^{\lambda t} \widehat{R}(\lambda) \widehat{N}(\lambda) d \lambda=\frac{1}{2 \pi i} \int_{\Gamma\left(r_{0}\right)} \lambda e^{\lambda t} \widehat{R}(\lambda) \widehat{N}(\lambda) d \lambda
$$

Para mostrar 4.20, introduzimos as curvas

$$
\begin{aligned}
\gamma_{1}^{n} & =\left\{s+i n:-n \operatorname{sen}\left(\phi-\frac{\pi}{2}\right) \leq s \leq \gamma\right\}, \\
\gamma_{2}^{n} & =\left\{-s-i n:-n \operatorname{sen}\left(\phi-\frac{\pi}{2}\right) \leq s \leq \gamma\right\}, \\
\gamma^{n} & =\{\gamma+i s:-n \leq s \leq n\} .
\end{aligned}
$$

No que segue assumiremos que a curvas $\Lambda_{n}=\Gamma\left(r_{0}\right) \bigcap\{\lambda ;|\lambda| \leq n\} \bigcup \gamma_{1}^{n} \bigcup \gamma_{2}^{n} \bigcup \gamma^{n}, n \in \mathbb{N}$, estão orientadas de modo de preservar a orientação em $\Gamma\left(r_{0}\right)$. Da analiticidade de $\lambda \rightarrow$ $\lambda e^{\lambda t} \widehat{R}(\lambda) \widehat{N}(\lambda)$ em $\Lambda_{r_{1}, \theta}$ e do Teorema de Cauchy é claro que

$$
\frac{1}{2 \pi i} \int_{\Lambda_{n}} \lambda e^{\lambda t} \widehat{R}(\lambda) \widehat{N}(\lambda) x d \lambda=0
$$

para todo $n \in \mathbb{N}$. Por outro lado, usando as estimativas do Lema 4.2 .2 e o fato que $\widehat{R}(\lambda)=$ 
$G(\lambda)$, para $\lambda \in \Lambda_{r_{1}, \theta}$, segue que

$$
\begin{aligned}
\left\|\int_{\gamma_{1}^{n}} \lambda e^{\lambda t} \widehat{R}(\lambda) \widehat{N}(\lambda) x d \lambda\right\| & +\left\|\int_{\gamma_{2}^{n}} \lambda e^{\lambda t} \widehat{R}(\lambda) \widehat{N}(\lambda) x d \lambda\right\| \\
& \leq 2 \int_{-n \operatorname{sen}\left(\phi-\frac{\pi}{2}\right)}^{\gamma} e^{s t} \sqrt{s^{2}+n^{2}} \frac{M_{3} N}{\left(\sqrt{s^{2}+n^{2}}\right)^{1+\alpha}}\|x\| d s \\
& \leq 2 \int_{-n \operatorname{sen}\left(\phi-\frac{\pi}{2}\right)}^{\gamma} e^{s t} \frac{M_{3} N}{\left(\sqrt{s^{2}+n^{2}}\right)^{\alpha}}\|x\| d s \\
& \leq 2 \int_{-n \operatorname{sen}\left(\phi-\frac{\pi}{2}\right)}^{\gamma} e^{s t} \frac{M_{3} N}{n^{\alpha}}\|x\| d s \\
& \leq 2 \frac{M_{3} N}{n^{\alpha}}\left(\frac{e^{\gamma t}}{t}-\frac{e^{-n t \operatorname{sen}\left(\phi-\frac{\pi}{2}\right)}}{t}\right)\|x\|,
\end{aligned}
$$

de onde obtemos que

$$
\lim _{n \rightarrow \infty} \frac{1}{2 \pi i} \int_{\gamma_{i}^{n}} \lambda e^{\lambda t} \widehat{R}(\lambda) \widehat{N}(\lambda) d \lambda=0, \quad i=1,2 .
$$

Agora de 4.21) e 4.22 inferimos que 4.20 é válida e assim que 4.18) é satisfeita para todo $t>0$.

De maneira similar podemos mostrar que 4.19 é também verificada para $t>0$. Para isto, definamos a função $\Theta_{1}:[0, \infty) \rightarrow X$ por $\Theta_{1}(s)=\int_{0}^{s} R(s-\mu) B(\mu) x d \mu$. Como $B(\cdot) x \in$ $L^{1}\left(\mathbb{R}^{+}: X\right)$, quando $x \in D(A)$, é claro que $\Theta_{1} \in C_{b}([0, \infty): X)$ pois

$$
\left\|\Theta_{1}(s)\right\| \leq M\|b\|_{L^{1}\left(\mathbb{R}^{+}\right)}\|x\|_{D(A)},
$$

para todo $s \geq 0$. Seja agora $\xi_{1}(s)=\int_{0}^{s} \Theta_{1}(\mu) d \mu$. Nestas condições a Transformada de Laplace-Stieltjes de $\xi_{1}$ existe para $\operatorname{Re}(\lambda)>0$ e

$$
\int_{0}^{\infty} e^{-\lambda t} d \xi_{1}(t)=\int_{0}^{\infty} e^{-\lambda t} \Theta_{1}(t) d t=\widehat{R}(\lambda) \widehat{B}(\lambda) x
$$

Pelo Teorema da Transformada de Laplace inversa [48, Theorem 6.3.1] e da definição de $\xi_{1}(\cdot)$, vemos que para $\gamma>r_{0}$

$$
\xi_{1}(t)=\frac{1}{2 \pi i} \int_{\gamma-i \infty}^{\gamma+i \infty} e^{\lambda t} \int_{0}^{\infty} e^{-\lambda t} d \xi_{1}(t) \frac{d \lambda}{\lambda}=\frac{1}{2 \pi i} \int_{\gamma-i \infty}^{\gamma+i \infty} e^{\lambda t} \widehat{R}(\lambda) \widehat{B}(\lambda) x \frac{d \lambda}{\lambda} .
$$

Do anterior segue que

$$
\Theta_{1}(t)=\frac{1}{2 \pi i} \int_{\gamma-i \infty}^{\gamma+i \infty} e^{\lambda t} \widehat{R}(\lambda) \widehat{B}(\lambda) x d \lambda
$$

Para finalizar esta parte da demonstração mostraremos que

$$
\frac{1}{2 \pi i} \int_{\gamma-i \infty}^{\gamma+i \infty} e^{\lambda t} \widehat{R}(\lambda) \widehat{B}(\lambda) d \lambda=\frac{1}{2 \pi i} \int_{\Gamma\left(r_{0}\right)} e^{\lambda t} \widehat{R}(\lambda) \widehat{B}(\lambda) d \lambda .
$$


Para isto, considere as curvas $\gamma_{1}^{n}, \gamma_{2}^{n}, \gamma^{n}$ e $\Lambda_{n}$ introduzidas anteriormente. Da analiticidade de $\lambda \rightarrow e^{\lambda t} \widehat{R}(\lambda) \widehat{B}(\lambda)$ em $\Lambda_{r_{1}, \theta}$ e do Teorema de Cauchy concluímos que

$$
\frac{1}{2 \pi i} \int_{\Lambda_{n}} \lambda e^{\lambda t} \widehat{R}(\lambda) \widehat{N}(\lambda) x d \lambda=0,
$$

para todo $n \in \mathbb{N}$. Por outro lado, procedendo de forma semelhante ao o caso anterior inferimos que

$$
\lim _{n \rightarrow \infty} \frac{1}{2 \pi i} \int_{\gamma_{i}^{n}} e^{\lambda t} \widehat{R}(\lambda) \widehat{B}(\lambda) d \lambda=0, \quad i=1,2 .
$$

Agora de 4.24 e 4.25 inferimos que 4.23 é válida e assim que 4.18 é satisfeita para $t>0$.

O passo feito anteriormente mostra que $(R(t))_{t \geq 0}$ verifica a equação 4.4 para todo $t>0$.

Vejamos agora que 4.3 também é válida. Usando que

$$
\lambda G(\lambda) x=[I-(\lambda \widehat{N}(\lambda)-\widehat{B}(\lambda)-A) G(\lambda)] x,
$$

e a integrabilidade das funções $\lambda \rightarrow e^{\lambda t} G(\lambda) A x, \lambda \rightarrow \lambda e^{\lambda t} \widehat{N}(\lambda) \widehat{R}(\lambda) x$ e $\lambda \rightarrow e^{\lambda t} \widehat{B}(\lambda) \widehat{R}(\lambda) x$ sobre $\Gamma\left(r_{0}\right)$, vemos que

$$
\begin{aligned}
& R^{\prime}(t) x \\
& \quad=\frac{1}{2 \pi i} \int_{\Gamma\left(r_{0}\right)} \lambda e^{\lambda t} G(\lambda) x d \lambda \\
& \quad=\frac{1}{2 \pi i} \int_{\Gamma\left(r_{0}\right)} e^{\lambda t}[x-(\lambda \widehat{N}(\lambda)-\widehat{B}(\lambda)-A) G(\lambda) x] d \lambda \\
& \quad=\frac{1}{2 \pi i} \int_{\Gamma\left(r_{0}\right)} e^{\lambda t} G(\lambda) A x d \lambda-\frac{1}{2 \pi i} \int_{\Gamma\left(r_{0}\right)} \lambda e^{\lambda t} \widehat{N}(\lambda) \widehat{R}(\lambda) x d \lambda+\frac{1}{2 \pi i} \int_{\Gamma\left(r_{0}\right)} e^{\lambda t} \widehat{B}(\lambda) \widehat{R}(\lambda) x d \lambda .
\end{aligned}
$$

Procedendo como antes, podemos mostrar que

$$
\begin{aligned}
\frac{1}{2 \pi i} \int_{\Gamma\left(r_{0}\right)} \lambda e^{\lambda t} \widehat{N}(\lambda) \widehat{R}(\lambda) x d \lambda & =\frac{d}{d t} \int_{0}^{t} N(t-s) R(s) x d s, \\
\frac{1}{2 \pi i} \int_{\Gamma\left(r_{0}\right)} e^{\lambda t} \widehat{B}(\lambda) \widehat{R}(\lambda) x d \lambda & =\int_{0}^{t} B(t-s) R(s) x d s,
\end{aligned}
$$

o que permite provar 4.3 . Como os detalhes são extensos e muito similares aos anteriores, omitiremos esta parte da demonstração.

É fácil ver dos passos anteriores, que as fuções $\Gamma_{1}(t)=R(t) x+\int_{0}^{t} R(t-s) N(s) x d s$ e $\Gamma_{2}(t)=R(t) x+\int_{0}^{t} N(t-s) R(s) x d s$ são de classe $C^{1}$ sobre $(0, \infty)$. Mostraremos agora que $\Gamma_{1}$ 
é continuamente diferenciável em zero e que (4.4) se verifica em zero. Para começar, observe que para $\epsilon>0$, existe $a>0$ tal que

$$
\left\|R(t) A x+\int_{0}^{t} R(t-s) N(s) x d s-A x\right\| \leq \epsilon, \quad t \in[0, a] .
$$

Se $\zeta \in X^{\prime}$, é claro que $\zeta \circ \Gamma_{1} \in C([0, a]: \mathbb{R}) \cap C^{1}((0, a): \mathbb{R})$. Assim, pelo Teorema do valor médio de Lagrange, para $t \in(0, a)$ existe $c_{\zeta, t} \in(0, t)$ tal que

$$
\begin{aligned}
\frac{\zeta \circ \Gamma_{1}(t)-\zeta \circ \Gamma_{1}(0)}{t} & =\left.\frac{d}{d t}\left(\zeta \circ \Gamma_{1}\right)\right|_{t=c_{\zeta, t}} \\
& =\zeta\left(\left.\frac{d}{d t} \Gamma_{1}\right|_{t=c_{\zeta, t}}\right) \\
& =\zeta\left(R\left(c_{\zeta, t}\right) A x+\int_{0}^{c_{\zeta, t}} R\left(c_{\varphi, t}-s\right) N(s) x d s\right) .
\end{aligned}
$$

O anterior, junto com 4.26, implica que

$$
\left|\frac{\zeta \circ \Gamma_{1}(t)-\zeta \circ \Gamma_{1}(0)}{t}-\zeta(A x)\right| \leq\|\zeta\|\left\|R\left(c_{\zeta, t}\right) A x+\int_{0}^{c_{\zeta, t}} R\left(c_{\zeta, t}-s\right) N(s) x d s-A x\right\|,
$$

o que mostra que

$$
\left\|\frac{\Gamma_{1}(t)-\Gamma_{1}(0)}{t}-A x\right\|=\sup _{\|\zeta\| \leq 1}\left|\frac{\zeta \circ \Gamma_{1}(t)-\zeta \circ \Gamma_{1}(0)}{t}-\zeta(A x)\right| \leq \epsilon,
$$

para todo $t \in[0, a]$. Por tanto, $\Gamma_{1}(\cdot)$ é diferenciável em 0 e $\lim _{t \downarrow 0} \frac{d}{d t} \Gamma_{1}(t)=\left.\frac{d}{d t} \Gamma_{1}(t)\right|_{t=0}=A x$, o que implica que $\Gamma_{1}(\cdot) \in C^{1}([0, \infty) ; X)$ e que 4.4 também se verifica em $t=0$.

A prova que $\Gamma_{2}(\cdot) \in C^{1}([0, \infty) ; X)$ e que 4.3 é válida em $t=0$ é similar à anterior e por isso será omitida. A demostração está agora completa.

Lema 4.2.11 A familia $(R(t))_{t \geq 0}$ possui uma extensão analítica na região

$$
\Lambda_{\delta}=\{t \in \mathbb{C}:|\arg (t)|<\delta\} \backslash\{0\}, \text { onde } 0<\delta<\phi-\frac{\pi}{2}<\frac{\pi}{2} .
$$

Demonstração: Primeiro mostraremos que a família de operadores definida em 4.13 converge em $\mathcal{L}(X)$ para todo $t \in \Lambda_{\delta}$.

Para $\lambda \in \Gamma_{r_{0}}^{1}$, temos que $\lambda t=|\lambda t| e^{i(\arg (\lambda)+\arg (t))}$, onde $\arg (\lambda)=\phi \mathrm{e}-\delta<\arg (t)<\delta$. Assim segue que

$$
\begin{array}{r}
\phi-\delta<\arg (t)+\arg (\lambda)<\phi+\delta \Rightarrow \\
\phi-\delta+\frac{\pi}{2}-\frac{\pi}{2}<\arg (t)+\arg (\lambda)<\phi+\delta+\left(\phi-\frac{\pi}{2}\right)-\left(\phi-\frac{\pi}{2}\right) \Rightarrow \\
\frac{\pi}{2}+\epsilon<\arg (t)+\arg (\lambda)<\frac{3 \pi}{2}-\epsilon,
\end{array}
$$


onde $\epsilon=\phi-\frac{\pi}{2}-\delta>0$. Como $\cos \left(\frac{\pi}{2}+\epsilon\right)=\cos \left(\frac{3 \pi}{2}-\epsilon\right)=-\operatorname{sen}(\epsilon)$, obtemos que

$$
\left|e^{\lambda t}\right| \leq e^{|\lambda t| \cos (\arg (t)+\arg (\lambda))} \leq e^{|\lambda t| \cos \left(\frac{\pi}{2}+\varepsilon\right)}=e^{-|\lambda t| \operatorname{sen}(\varepsilon)} .
$$

Para $\lambda \in \Gamma_{r_{0}}^{3}$ vemos que $\lambda t=|\lambda t| e^{i(-\arg (\lambda)+\arg (t))}$, onde $\arg (\lambda)=\phi \mathrm{e}-\delta<\arg (t)<\delta$. Logo obtemos que $\frac{-3 \pi}{2}+\varepsilon<\arg (t)-\arg (\lambda)<\frac{-\pi}{2}-\varepsilon$, e que $\cos \left(\frac{-3 \pi}{2}+\varepsilon\right)=\cos \left(\frac{-\pi}{2}-\varepsilon\right)=$ $-\operatorname{sen}(\varepsilon)$. Conseqüêntemente,

$$
\left|e^{\lambda t}\right| \leq e^{|\lambda t| \cos (\arg (t)-\arg (\lambda))} \leq e^{|\lambda t| \cos \left(\frac{-\pi}{2}+\varepsilon\right)}=e^{-|\lambda t| \operatorname{sen}(\varepsilon)} .
$$

Quando $\lambda \in \Gamma_{r_{0}}^{2}$, é fácil ver que

$$
\left|e^{\lambda t}\right| \leq e^{|t| r_{0} \cos (\phi+\arg (t))} \leq e^{|t| r_{0}} .
$$

Procedendo agora como no Lema 4.2.3, usando 4.27)- 4.29 podemos mostrar que a família de operador $(R(t))_{t \in \Lambda_{\delta}} \in \mathcal{L}(X)$. Mais ainda, usando as idéias da prova do Lema 4.2.6 podemos mostrar que $R^{\prime}(\cdot) \in C\left(\Lambda_{\delta}, \mathcal{L}(X)\right)$, o que permite concluir a prova.

Como conseqüência dos Lemas 4.2.3 4.2.11, estabelecemos sem demostração, o principal resultado desta seção.

Teorema 4.2.1 Se as condições $\left(\mathbf{H N}_{\mathbf{1}}\right)-\left(\mathbf{H N}_{\mathbf{6}}\right)$ são satisfeitas, então a família $(R(t))_{t \geq 0}$ é uma familia $N$-resolvente analítica para (4.1)-(4.2).

Corolário 4.2.1 Suponha que as condições $\left(\mathbf{H N}_{\mathbf{1}}\right)-\left(\mathbf{H N}_{\mathbf{6}}\right)$ são satisfeitas. Então existem cons- tantes positivas $C_{1}, C_{2}$ tais que

$$
\left\|R^{\prime}(t) x\right\| \leq\left(C_{1}+C_{2} t^{\alpha-1}\right)\|x\|_{[D(A)]}
$$

para todo $x \in D(A)$.

Demonstração: Sejam $t>0$ e $x \in D(A)$. Como $\lambda G(\lambda) x=G(\lambda)(A-\lambda \widehat{N}(\lambda)+\widehat{B}(\lambda)) x$, do Lema 4.2 .6 segue que

$$
R^{\prime}(t) x=\frac{1}{2 \pi i} \int_{\Gamma} e^{\lambda t} G(\lambda)(A-\lambda \widehat{N}(\lambda)+\widehat{B}(\lambda)) x d \lambda .
$$

Para estabelecer a desigualdade 4.30 , estudaremos os casos $t \in(0,1)$ e $t \geq 1$. Assuma primeiramente que $t \in(0,1)$. Se $M_{3}$ é a constante garantida pelo Lema 4.2.2, da condição 
$\left(\mathbf{H N}_{\mathbf{5}}\right)$ e da analiticidade de $\lambda \rightarrow G(\lambda)(A-\lambda \widehat{N}(\lambda)+\widehat{B}(\lambda))$ obtemos que

$$
\begin{aligned}
\left\|R^{\prime}(t) x\right\| & \leq \frac{1}{2 \pi} \int_{\Gamma\left(t^{-1} r_{0}\right)} e^{|t \lambda|}\|G(\lambda)(A-\lambda \widehat{N}(\lambda)+\widehat{B}(\lambda)) x\||d \lambda| \\
\leq & \frac{1}{2 \pi} \int_{\Gamma\left(t^{-1} r_{0}\right)} e^{|t \lambda|} \frac{M_{3}}{|\lambda|}\left(I+|\lambda| \frac{N}{|\lambda|^{\alpha}}+\widehat{b}(\lambda)\right)\|x\|_{[D(A)]}|d \lambda| \\
\leq & \frac{1}{\pi} \int_{\Gamma\left(t^{-1} r_{0}\right)} e^{|t \lambda|} \frac{M_{3}\left(1+\frac{1}{M_{2}}\right)}{|\lambda|}\|x\|_{[D(A)]}|d \lambda|+\frac{1}{2 \pi} \int_{\Gamma\left(t^{-1} r_{0}\right)} e^{|t \lambda|} \frac{M_{3} N}{|\lambda| \alpha}\|x\|_{[D(A)]}|d \lambda| \\
= & \frac{2}{\pi} \int_{t^{-1} r_{0}}^{\infty} e^{-r t \operatorname{sen}\left(\phi-\frac{\pi}{2}\right)} \frac{M_{3}\left(1+\frac{1}{M_{2}}\right)}{r} d r\|x\|_{[D(A)]} \\
& +\frac{1}{\pi} \int_{-\phi}^{\phi} e^{t^{-1} r_{0} t \cos \xi} M_{3}\left(1+\frac{1}{M_{2}}\right) d \xi\|x\|_{[D(A)]} \\
& +\frac{1}{\pi} \int_{t^{-1} r_{0}}^{\infty} e^{-r t \operatorname{sen}\left(\phi-\frac{\pi}{2}\right)} \frac{M_{3} N}{r^{\alpha}} d r\|x\|_{[D(A)]}+\frac{1}{2 \pi} \int_{-\phi}^{\phi} e^{t^{-1} r_{0} t \cos \xi} \frac{M_{3} N}{r^{\alpha}} r d \xi\|x\|_{[D(A)]} \\
= & M_{3}\left(1+\frac{1}{M_{2}}\right)\left(\frac{2}{\pi r_{0}} \frac{e^{-r_{0} \operatorname{sen}\left(\phi-\frac{\pi}{2}\right)}}{\operatorname{sen}\left(\phi-\frac{\pi}{2}\right)}+\frac{1}{\pi} e^{r_{0} \phi}\right)\|x\|_{[D(A)]} \\
& +\left(\frac{t^{\alpha} M_{3} N}{\pi r_{0}^{\alpha}} \frac{e^{-r_{0} \operatorname{sen}\left(\phi-\frac{\pi}{2}\right)}}{t \operatorname{sen}\left(\phi-\frac{\pi}{2}\right)}+\frac{M_{3} N}{\pi r_{0}^{\alpha-1}} t^{\alpha-1} e^{r_{0}} \phi\right)\|x\|_{[D(A)]},
\end{aligned}
$$

assim obtemos que

$$
R^{\prime}(t) x \leq\left(C_{1}+C_{2} t^{\alpha-1}\right)\|x\|_{[D(A)]},
$$

onde $C_{1}$ e $C_{2}$ são constantes independentes de $t$ e $x$.

Vejamos agora o caso $t \geq 1$. Usando a mudança de variável $\lambda t=\gamma$, fazendo uso da analiticidade da função $\lambda \rightarrow G(\lambda)(A-\lambda \widehat{N}(\lambda)+\widehat{B}(\lambda))$ e do Lema 4.2 .2 obtemos que

$$
\begin{aligned}
\| & R^{\prime}(t) x \| \\
& \leq \frac{1}{2 \pi} \int_{\Gamma\left(r_{0}\right)} e^{|\gamma|}\left\|G\left(t^{-1} \gamma\right)\left(A-t^{-1} \lambda \widehat{N}\left(t^{-1} \gamma\right)+\widehat{B}\left(t^{-1} \gamma\right)\right) x\right\| t^{-1}|d \gamma| \\
& \leq \frac{1}{2 \pi} \int_{\Gamma\left(r_{0}\right)} e^{|\gamma|} \frac{M_{3}}{|\gamma| t^{-1}}\left(I+|\gamma| t^{-1} \frac{M_{3} N}{|\gamma|^{\alpha} t^{-\alpha}}+\widehat{b}\left(t^{-1} \gamma\right)\right)\|x\|_{[D(A)]} t^{-1}|d \gamma| \\
& \leq \frac{1}{\pi} \int_{\Gamma\left(r_{0}\right)} e^{|\gamma|} \frac{M_{3}\left(1+\frac{1}{M_{2}}\right)}{|\gamma|}\|x\|_{[D(A)]}|d \gamma|+\left(\frac{1}{2 \pi} \int_{\Gamma\left(r_{0}\right)} e^{|\gamma|} \frac{M_{3} N}{|\gamma|^{\alpha}}\|x\|_{[D(A)]}|d \gamma|\right) t^{\alpha-1}
\end{aligned}
$$

de onde inferimos a existência de $C_{3}$ e $C_{4}$ independentes de $t$ e $x$ tais que

$$
\left\|R^{\prime}(t) x\right\| \leq\left(C_{3}+C_{4} t^{\alpha-1}\right)\|x\|_{[D(A)]} .
$$

A propriedade agora é conseqüência de 4.31) e 4.32). 
Corolário 4.2.2 Assuma que $A$ é o gerador infinitesimal de um semigrupo analítico; $0 \in$ $\rho(A)$ e que para todo $\vartheta \in(0,1)$ o operador $\lambda \rightarrow(-A)^{\vartheta} G(\lambda)$ é analítico sobre $\Lambda_{r_{1}, \theta}$. Então, para cada $\vartheta \in(0,1)$ existe $K_{\vartheta}>0$ tal que

$$
\left\|(-A)^{\vartheta} R(t)\right\| \leq \frac{K_{\vartheta}}{t^{\vartheta}}, \quad t>0 .
$$

Demonstração: Seja $\vartheta \in(0,1)$. De [63, Theorem 6.10] sabemos que existe $C_{\vartheta}>0$ tal que

$$
\left\|(-A)^{\vartheta} x\right\| \leq C_{\vartheta}\|A x\|^{\vartheta}\|x\|^{1-\vartheta}, \quad x \in D(A) .
$$

Como $G(\cdot)$ assume valores em $D(A)$, do Lemma 4.2 .2 vemos que para $x \in X$

$$
\begin{aligned}
\left\|(-A)^{\vartheta} G(\lambda) x\right\| & \leq C_{\vartheta}\|A G(\lambda) x\|^{\vartheta}\|G(\lambda) x\|^{1-\vartheta} \\
& \leq C_{\vartheta} M_{5}^{\vartheta}\|x\|^{\vartheta} \frac{M_{3}^{1-\vartheta}}{\|\lambda\|^{1-\vartheta}}\|x\|^{1-\vartheta} \\
& \leq \frac{C_{1}}{\|\lambda\|^{1-\vartheta}}\|x\|,
\end{aligned}
$$

onde $C_{1}$ é independente de $\lambda$. A desigualdade anterior permite mostrar com facilidade a funcão $\lambda \rightarrow e^{\lambda t}(-A)^{\vartheta} G(\lambda)$ é integrável sobre $\Gamma(r), r \geq r_{0}$.

Como $(-A)^{\vartheta}$ é fechado e $(-A)^{\vartheta} G(\lambda)$ é analítica em $\Lambda_{r_{1}, \theta}$, para $t \in(0,1)$ obtemos que

$$
(-A)^{\vartheta} R(t) x=\frac{1}{2 \pi i} \int_{\Gamma\left(t^{-1} r_{0}\right)} e^{\lambda t}(-A)^{\vartheta} G(\lambda) x d \lambda .
$$

Usando a representação anterior, segue que

$$
\begin{aligned}
\| & (-A)^{\vartheta} R(t) x \| \\
& \leq \frac{1}{\pi} \int_{t^{-1} r_{0}}^{\infty}\left|e^{\lambda t}\right| \frac{C_{1}}{|\lambda|^{1-\vartheta}}|d \lambda|\|x\|+\frac{1}{2 \pi} \int_{-\phi}^{\phi}\left|e^{\lambda t}\right| \frac{C_{1}}{\left.|\lambda|\right|^{1-\vartheta}}|d \lambda|\|x\| \\
& \leq \frac{1}{\pi} \int_{t^{-1} r_{0}}^{\infty} e^{-r \operatorname{sen}\left(\phi-\frac{\pi}{2}\right)} \frac{C_{1}}{r^{1-\vartheta}} d r\|x\|+\frac{1}{2 \pi} \int_{-\phi}^{\phi} e^{t^{-1} r_{0} t \cos \xi} \frac{C_{1}}{t^{-1} r_{0}^{1-\vartheta}} t^{-1} r_{0} d \xi\|x\| \\
& \leq\left(\frac{C_{1}}{\pi r_{0}^{1-\vartheta}} \frac{e^{-r_{0} \operatorname{sen}\left(\phi-\frac{\pi}{2}\right)}}{\operatorname{sen}\left(\phi-\frac{\pi}{2}\right)}+\frac{C_{1}}{\pi} e^{r_{0}} \phi\right) t^{-\vartheta}\|x\|,
\end{aligned}
$$

de onde concluímos que existe $C_{2}>0$ independente de $x$ tal que

$$
\left\|(-A)^{\vartheta} R(t) x\right\| \leq C_{2} t^{-\vartheta}, \quad t \in(0,1) .
$$

Vejamos agora o caso em que $t \geq 1$. Fazendo a mudança de coordenada $\gamma=\lambda t$ e o fato que $(-A)^{\vartheta} G(\lambda)$ é analítica em $\Lambda_{r_{1}, \theta}$ obtemos que

$$
(-A)^{\vartheta} R(t) x=\frac{1}{2 \pi i} \int_{\Gamma\left(r_{0}\right)} e^{\gamma}(-A)^{\vartheta} G\left(\gamma t^{-1}\right) x t^{-1} d \gamma
$$


Do anterior segue que

$$
\begin{aligned}
\| & (-A)^{\vartheta} R(t) x \| \\
& \leq \frac{1}{\pi} \int_{r_{0}}^{\infty} t^{-1}\left|e^{\gamma}\right| \frac{C_{1}}{\left|t^{-1} \gamma\right|^{1-\vartheta}}|d \gamma|\|x\|+\frac{1}{2 \pi} \int_{-\phi}^{\phi} t^{-1}\left|e^{\gamma}\right| \frac{C_{1}}{\left|t^{-1} \gamma\right|^{1-\vartheta}}|d \gamma|\|x\| \\
& \leq \frac{1}{\pi} \int_{r_{0}}^{\infty} e^{-r \operatorname{sen}\left(\phi-\frac{\pi}{2}\right)} \frac{C_{1}}{r^{1-\vartheta}} t^{-\vartheta} d r\|x\|+\frac{1}{2 \pi} \int_{-\phi}^{\phi} e^{r_{0} \cos \xi} \frac{C_{1}}{r_{0}^{1-\vartheta}} t^{-\vartheta} r_{0} d \xi\|x\| \\
& \leq\left(\frac{C_{1}}{\pi r_{0}^{1-\vartheta}} \frac{e^{-r_{0} \operatorname{sen}\left(\phi-\frac{\pi}{2}\right)}}{\operatorname{sen}\left(\phi-\frac{\pi}{2}\right)}+\frac{C_{1}}{\pi} e^{r_{0}} \phi\right) t^{-\vartheta}\|x\|,
\end{aligned}
$$

de onde obtemos que

$$
\left\|(-A)^{\vartheta} R(t) x\right\| \leq C_{2} t^{-\vartheta}, \quad t \geq 1
$$

A conclusão do resultado é conseqüência de 4.34-4.35. A prova está concluída.

Para finalizar esta seção consideramos algums exemplos de sistemas integrodiferenciais que podem ser estudados sob nossa teoria de resolventes.

Exemplo 4.2.1 Considere o sistema integro-diferencial

$$
\begin{aligned}
\frac{\partial}{\partial t}\left[u(t, \xi)+\int_{0}^{t}(t-s)^{\alpha-1} e^{-\omega(t-s)} u(s, \xi) d s\right] & =\frac{\partial^{2}}{\partial \xi^{2}}\left[u(t, \xi)+\int_{0}^{t} e^{-\gamma(t-s)} u(s, \xi) d s\right], t>0, \\
u(0, \xi) & =0, \quad \xi \in[0, \pi]
\end{aligned}
$$

onde $\alpha \in(0,1)$ e $\omega, \gamma$ são números positivos. No que segue $X=L^{2}([0, \pi])$ e $A: D(A) \subset$ $X \rightarrow X$ é o operador definido por $A f(\xi)=f^{\prime \prime}(\xi)$, onde

$$
D(A)=\left\{f \in L^{2}([0, \pi]): f^{\prime \prime} \in L^{2}([0, \pi]), \quad f(0)=f(\pi)=0\right\} .
$$

É conhecido que A é o gerador infinitesimal de um semigrupo analítico e compacto $(T(t))_{t \geq 0}$ sobre $X$. Se definirmos os operadores $N_{1}(t) f(\xi)=t^{\alpha-1} e^{-\omega t} f(\xi)$ e $B_{1}(t) f(\xi)=b_{1}(t) f^{\prime \prime}(\xi)$ em que $b_{1}(t)=e^{-\gamma t}$, então podemos representar o sistema (4.36)- (4.37) na forma abstrata

$$
\begin{aligned}
\frac{\partial}{\partial t}\left[u(t)+\int_{0}^{t} N_{1}(t-s) u(s) d s\right] & =A u(t)+\int_{0}^{t} B_{1}(t-s) u(s) d s, \quad t>0 \\
u(0) & =0 .
\end{aligned}
$$

É fácil ver que as condições $\left(\mathbf{H N}_{\mathbf{1}}\right)$ e $\left(\mathbf{H N}_{\mathbf{2}}\right)$ são verificadas. Em relação a $\left(\mathbf{H N}_{\mathbf{3}}\right)$ e $\left(\mathbf{H N}_{4}\right)$ somente observamos $\|B(t) f\| \leq b_{1}(t)\|f\|_{[D(A)]} ;$ sendo $\widehat{b}_{1}(\lambda)=\frac{1}{\lambda+\gamma}$ e $\lim _{|\lambda| \rightarrow \infty} \widehat{b}_{1}(\lambda)=$ 0 e a condição $\left(\mathbf{H N}_{4}\right)$ é verificada se considerarmos $D=C_{0}^{\infty}([0, \pi])$.

Como conseqüência dos fatos anteriores temos a seguintes proposição: 
Proposição 4.2.1 Existe uma família de operadores $N$-resolvente associada ao sistema (4.36)(4.37).

Demonstração: Conseqüência imediata do Teorema 4.2.1.

Exemplo 4.2.2 Observe que a Proposição 4.2.1 também é verdadeira para o sistema

$$
\begin{aligned}
\frac{\partial}{\partial t}\left[u(t, \xi)+\int_{0}^{t} k(t-s) u(s, \xi) d s\right] & =\frac{\partial^{2}}{\partial \xi^{2}} u(t, \xi)+\int_{0}^{t} e^{-\gamma(t-s)} \frac{\partial}{\partial \xi} u(s, \xi) d s \\
u(0, \xi) & =0 \quad \xi \in[0, \pi] .
\end{aligned}
$$

onde $\alpha \in(0,1), \omega, \gamma>0$ e o núcleo $k(\cdot)$ é dado por

$$
k(t)=\left\{\begin{array}{cr}
t^{\alpha-1} & 0<t<1, \\
t^{\alpha-1} e^{-\omega t} & t \geq 1
\end{array}\right.
$$

\subsection{O problema integro-diferencial não homogêneo}

Fazendo uso da teoria desenvolvida na seção anterior, estudamos a existência e regularidade das soluções para o problema não homogêneo.

$$
\begin{aligned}
\frac{d}{d t}\left(x(t)+\int_{0}^{t} N(t-s) x(s) d s\right) & =A x(t)+\int_{0}^{t} B(t-s) x(s) d s+f(t), \quad t \in[0, a], \\
x(0) & =x_{0} \in D(A)
\end{aligned}
$$

onde $f:[0, a] \rightarrow X$ é apropriada. No que segue, sempre assumiremos que as condições $\left(\mathbf{H N}_{\mathbf{1}}\right)-\left(\mathbf{H N}_{\mathbf{4}}\right)$ são satisfeitas e denotaremos por $(R(t))_{t \geq 0}$ a família N-resolvente associada a 4.1)-(4.2). Além do anterior, assumiremos a seguinte propriedade

$\left(\mathbf{H}_{\alpha}\right)$ Existe $\alpha(\cdot) \in L_{l o c}^{1}\left(\mathbb{R}^{+}\right)$, a qual é limitada em intervalos da forma $\left(a_{1}, a_{2}\right], a_{1}>0$, tal que

$$
\left\|R^{\prime}(t) x\right\| \leq \alpha(t)\|x\|_{[D(A)]}, \forall x \in D(A) .
$$

Para o sistema integro-diferencial (4.42)-(4.43), consideremos a seguinte definição de solução clássica.

Definição 4.3.1 Uma função $x:[0, a) \rightarrow X$ é uma solução clássica de 4.42)-(4.43) se $x+N * x \in C^{1}((0, a): X) ; x \in C([0, a):[D(A)]) \cap C^{1}((0, a): X)$ e 4.42)-(4.43) é satisfeita sobre $[0, a)$.

Uma conseqüência imediata da existência de uma família de operadores N-resolvente para 4.1)-4.2 é a formula da variação dos parâmetros. 
Teorema 4.3.1 Seja $u_{0} \in D(A)$ e $f \in C([0, a]: X)$. Se u(·) é uma solução clássica de (4.42)-(4.43) e u'(.) é limitada sobre $(0, a]$, então

$$
u(t)=R(t) u_{0}+\int_{0}^{t} R(t-s) f(s) d s, \quad t \in[0, a] .
$$

Demonstração: Para começar, estudaremos a diferenciabilidade das funções

$$
\begin{aligned}
\Theta_{1}(s) & =\int_{0}^{s} N(s-\xi) u(\xi) d \xi \\
\Theta_{2}(s) & =\int_{0}^{s} R(s-\xi) N(\xi) u_{0} d \xi
\end{aligned}
$$

Para $s \in[0, a)$ e $h>0$ tal que $s+h \in[0, a]$ temos que

$$
\begin{aligned}
\frac{1}{h} & {\left[\Theta_{1}(s+h)-\Theta_{1}(s)\right] } \\
& =\int_{0}^{s} N(s-\xi) \frac{u(\xi+h)-u(\xi)}{h} d \xi+\frac{1}{h} \int_{0}^{h} N(s+h-\xi) u(\xi) d \xi .
\end{aligned}
$$

É fácil ver que o segundo termo da direita em 4.44 converge para $N(s) u_{0}$ q.t.p. sobre $[0, a]$. Por outro lado, como $u^{\prime}(\cdot)$ é limitada em $(0, a]$ e $N(\cdot)$ é integrável, concluímos que $\Theta_{1}$ é diferenciável em $s$ e que

$$
\Theta_{1}^{\prime}(s)=\int_{0}^{s} N(s-\xi) u^{\prime}(\xi) d \xi+N(s) u_{0} \text {, q.t.p. para } s \in[0, a] .
$$

Similarmente, para $u_{0} \in D(A), s \in[0, a)$ e $h>0$ tal que $s+h \in[0, a]$ vemos que

$$
\begin{aligned}
\frac{1}{h}\left[\Theta_{2}(s+h)-\Theta_{2}(s)\right]= & \int_{0}^{s}\left(\frac{R(s+h-\xi)-R(s-\xi)}{h}\right) N(\xi) u_{0} d \xi \\
& +\frac{1}{h} \int_{s}^{s+h} R(s+h-\xi) N(\xi) u_{0} d \xi .
\end{aligned}
$$

Como $N(\cdot) \in L^{1}([0, \infty): \mathcal{L}(X))$, segue facilmente que o segundo termo da direita em 4.45, converge q.t.p. para $N(s) u_{0}$ quando $h \rightarrow 0$. Mais ainda, como $N(t) D(A) \subset D(A)$ e a função $\mu \rightarrow R(\mu) N(\xi) u_{0}$ é diferenciável temos que

$$
\frac{R(s+h-\xi)-R(s-\xi)}{h} N(\xi) u_{0} \rightarrow R^{\prime}(s-\xi) N(\xi) u_{0},
$$

q.t.p. para $s \in[0, a]$. Usando agora a propriedade $\left(\mathbf{H}_{\alpha}\right)$ na definição de N-resolvente, e a desigualdade

$$
\begin{aligned}
\left\|\frac{R(s+h-\xi)-R(s-\xi)}{h} N(\xi) x\right\| & \leq\left\|\frac{1}{h} \int_{0}^{h} R^{\prime}(s-\xi+\theta) N(\xi) d \theta\right\| \\
& \leq \frac{1}{h} \int_{0}^{h} \alpha(s-\xi+\theta) d \theta\|N(\xi) x\|_{[D(A)]}=g(\xi),
\end{aligned}
$$


do Teorema da Convergência Dominada obtemos que a função $\xi \rightarrow R^{\prime}(s-\xi) N(\xi) x$ é integrável sobre $[0, s]$. Como conseqüência dos passos anteriores, $\Theta_{2}$ é diferenciável e

$$
\Theta_{2}^{\prime}(s)=\int_{0}^{s} R^{\prime}(s-\xi) N(\xi) x d \xi+N(s) x, \text { q.t.p. para } s \in[0, a] .
$$

Usando agora que $R(\cdot) u_{0} \in C^{1}((0, \infty): X)$, as equações N-resolventes, a diferenciabilidade de $\Theta_{1}, \Theta_{2}$ é o fato que $u(\cdot)$ é solução clássica segue que

$$
\begin{aligned}
u(t)- & R(t) u_{0}-\int_{0}^{t} R(t-s) f(s) d s \\
= & \int_{0}^{t} \frac{d}{d s}(R(t-s) u(s)) d s-\int_{0}^{t} R(t-s) f(s) d s \\
= & -\int_{0}^{t} R^{\prime}(t-s) u(s) d s+\int_{0}^{t} R(t-s) u^{\prime}(s) d s-\int_{0}^{t} R(t-s) f(s) d s \\
= & -\int_{0}^{t} R^{\prime}(t-s) u(s) d s+\int_{0}^{t} R(t-s)\left(A u(s)+\int_{0}^{s} B(s-\xi) u(\xi) d \xi+f(s)\right) d s \\
& -\int_{0}^{t} R(t-s) \frac{d}{d s}\left(\int_{0}^{s} N(s-\xi) u(\xi) d \xi\right) d s-\int_{0}^{t} R(t-s) f(s) d s \\
= & -\int_{0}^{t}\left(R(t-s) A+\int_{0}^{t-s} R(t-s-\xi) B(\xi) d \xi\right)^{t} u(s) d s \\
& +\int_{0}^{t} \frac{d}{d s}\left(\int_{0}^{t-s} R(t-s-\xi) N(\xi) d \xi\right) u(s) d s \\
& +\int_{0}^{t} R(t-s) A u(s) d s+\int_{0}^{t} R(t-s) \int_{0}^{s} B(s-\xi) u(\xi) d \xi d s \\
& -\int_{0}^{t} R(t-s) \frac{d}{d s}\left(\int_{0}^{s} N(s-\xi) u(\xi) d \xi\right) d s \\
= & \int_{0}^{t} \frac{d}{d s}\left(\int_{0}^{t-s} R(t-s-\xi) N(\xi) d \xi\right) u(s) d s-\int_{0}^{t} R(t-s) \frac{d}{d s}\left(\int_{0}^{s} N(s-\xi) u(\xi) d \xi\right) d s \\
= & \int_{0}^{t} \frac{d}{d s}\left(\int_{0}^{t-s} R(t-s-\xi) N(\xi) d \xi\right) u(s) d s \\
& -\left.R(t-s) \int_{0}^{s} N(s-\xi) u(\xi) d \xi\right|_{0} ^{t}+\int_{0}^{t} R^{\prime}(t-s) \int_{0}^{s} N(s-\xi) u(\xi) d \xi d s \\
= & \int_{0}^{t} \int_{0}^{t-s}-R^{\prime}(t-s-\xi) N(\xi) u(s) d \xi+\int_{0}^{t} N(t-s) u(s) d s \\
& -\int_{0}^{t} N(t-s) u(s) d s+\int_{0}^{t} R^{\prime}(t-s) \int_{0}^{s} N(s-\xi) u(\xi) d \xi d s \\
& \int_{0}^{\prime}(t-s-\xi) N(\xi) d \xi u(s) d s+\int_{0}^{t} R^{\prime}(t-s) \int_{0}^{s} N(s-\xi) u(\xi) d \xi d s=0, \\
& \\
&
\end{aligned}
$$

para cada $t \in[0, a]$. Isto prova que

$$
u(t)=R(t) u_{0}+\int_{0}^{t} R(t-s) f(s) d s, \quad t \in[0, a] .
$$

O que completa a demonstração. 
Corolário 4.3.1 Existe uma única família $N$-resolvente associada ao sistema (4.1)- (4.2).

Demonstração: Assuma que $\left(R_{1}(t)\right)_{t \geq 0}$ é uma outra família N-resolvente para (4.1)-(4.2). Se $x \in D(A)$, a função $u(t)=R_{1}(t) x$ é uma solução clássica de 4.42)-4.43 com $f \equiv 0$. Agora do Teorema 4.3.1 segue que que $R_{1}(t) x=R(t) x$ para todo $x \in D(A)$. Como $D(A)$ é denso em $X$ e $R_{1}(\cdot), R(\cdot)$ são limitadas sobre compactos de $\mathbb{R}$ segue que $R_{1}(t)=R_{2}(t)$ para todo $t \geq 0$.

Motivados pelo Teorema 4.3.1, introduzimos o seguinte conceito de solução fraca para (4.42)-4.43).

Definição 4.3.2 Seja $f \in L^{1}([0, a]: X)$. A função $u \in C([0, a]: X)$ definida por

$$
u(t)=R(t) x+\int_{0}^{t} R(t-s) f(s) d s, \quad t \in[0, a] .
$$

é chamada solução fraca de 4.42)-(4.43).

Para estudar condições sob as quais uma solução fraca é clássica, precisamos do seguinte Lema.

Lema 4.3.1 Seja $V(\cdot) \in C([0, \infty): \mathcal{L}(X))$ a função definida por $V(t)=\int_{0}^{t} R(s) d s$. Então $V(t) X \subset D(A)$ para todo $t \geq 0$ e $A V(\cdot) x \in C([0, a]: X)$ para todo a $>0$ e todo $x \in X$.

Demonstração: Sejam $x \in D(A)$ e $a>0$. Integrando a equação N-resolvente (4.3), obtemos que

$$
R(t) x+\int_{0}^{t} N(t-s) R(s) x d s-x=\int_{0}^{t} A R(s) x d s+\int_{0}^{t} \int_{0}^{s} B(s-\xi) R(\xi) x d \xi d s .
$$

Como $A$ é fechado e $A R(\cdot) x$ é integrável obtemos do fato anterior que

$$
A V(t) x=R(t) x+\int_{0}^{t} N(t-s) R(s) x d s-x-\int_{0}^{t} B(t-s) V(s) x d s, \quad t \in[0, a],
$$

o que implica que $A V(\cdot) x \in C([0, a]: X)$ se $x \in D(A)$.

Para estudar o caso geral precisamos de uma estimativa para $A V(\cdot) x$ em $X$.

Seja $\phi(t)=\|V(t) x\|_{[D(A)]}$, de (4.47) segue que

$\phi(t)$

$$
\begin{aligned}
& \leq\|V(t) x\|+\|A V(t)\| \\
& \leq\left\|\int_{0}^{t} R(s) x d s\right\|+\|R(t) x\|+\left\|\int_{0}^{t} N(t-s) R(s) x d s\right\|+\|x\|+\left\|\int_{0}^{t} B(t-s) V(s) d s\right\| \\
& \leq M a\|x\|+M\|x\|+M\|x\| \int_{0}^{a}\|N(s)\| d s+\|x\|+\int_{0}^{t}\|B(t-s) V(s)\| d s,
\end{aligned}
$$


assim

$$
\phi(t) \leq c_{1}\|x\|+\int_{0}^{t} b(t-s) \phi(s) d s
$$

onde $c_{1}>0$ é uma constante independente de $t \in[0, a]$ e $x \in D(A)$. Sejam $0<t_{1}<t_{2}<$ $\ldots<t_{n}=a$ tais que $\int_{t_{i}}^{t_{i+1}} b(s) d s \leq \frac{1}{2}$ para cada $i=1 \ldots n$. Para $t \in\left[0, t_{1}\right]$, da desigualdade (4.48) segue que

$$
\phi(t) \leq c_{1}\|x\|+\sup _{s \in\left[0, t_{1}\right]} \phi(s) \int_{0}^{t} b(s) d s
$$

$\log 0$

$$
\sup _{s \in\left[0, t_{1}\right]} \phi(s) \leq c_{1}\|x\|+\frac{1}{2} \sup _{s \in\left[0, t_{1}\right]} \phi(s) .
$$

Como conseqüência,

$$
\sup _{s \in\left[0, t_{1}\right]} \phi(s) \leq 2 c_{1}\|x\|
$$

Usando 4.49, para $t \in\left[t_{1}, t_{2}\right]$ obtemos que

$$
\begin{aligned}
\phi(t) & \leq c_{1}\|x\|+\int_{0}^{t_{1}} b(t-s) \phi(s) d s+\int_{t_{1}}^{t} b(t-s) \phi(s) d s \\
& \leq c_{1}\|x\|+\|b\|_{L^{1}} 2 c_{1}\|x\|+\sup _{s \in\left[0, t_{2}\right]} \phi(s) \int_{0}^{t-t_{1}} b(s) d s \\
& \leq c_{1}\|x\|+\|b\|_{L^{1}} 2 c_{1}\|x\|+\frac{1}{2} \sup _{s \in\left[0, t_{2}\right]} \phi(s),
\end{aligned}
$$

e assim

$$
\sup _{s \in\left[0, t_{2}\right]} \phi(s) \leq 2 c_{1}\left(1+2\|b\|_{L^{1}}\right)\|x\| .
$$

Reinterando o processo anterior, deduzimos a existência de $c_{2}>0$, independente de $t \in[0, a]$ e $x \in D(A)$, tal que

$$
\sup _{s \in[0, a]} \phi(s) \leq c_{2}\|x\|
$$

Seja agora $x \in X$ e $\left(x_{n}\right)_{n \in \mathbb{N}}$ em $D(A)$ tal que $x_{n} \rightarrow x$ quando $n \rightarrow \infty$. Da desigualdade 4.50, é claro que a seqüência $\left(A V(t) x_{n}\right)_{n \in \mathbb{N}}$ é convergente para um ponto $y \in X$. Como $A$ é fechado, concluímos que $V(t) x \in D(A)$ e que $A V(t) x=y$. Mais ainda, é fácil ver que $A V(t) x_{n} \rightarrow A V(t) x$ uniformemente em $[0, a]$ o que mostra que $A V(\cdot) x \in C([0, a]: X)$.

O próximo Teorema é um clássico resultado de regularidade de soluções fracas. Especificamente, no Teorema 4.3.2, estabeleceremos condições de modo que uma solução fraca seja uma solução clássica. 
Teorema 4.3.2 Seja $u_{0} \in D(A)$ e $f \in C([0, a]: X)$. Se $f \in L^{1}([0, a]:[D(A)])$ ou $f \in$ $W^{1,1}([0, a]: X)$, então solução fraca de (4.42)-(4.43) é uma solução clássica de (4.42)-(4.43).

Demonstração: Seja $u(\cdot)$ a solução fraca de (4.42)-4.43). Considere inicialmente o caso em que $f \in C^{1}([0, a]:[D(A)])$ e assuma que $u_{0}=0$. Das propriedades da família $(R(t))_{t \geq 0}$, podemos mostrar que a função $\Lambda(t)=\int_{0}^{t} A R(t-s) f(s) d s$ é contínua sobre [0,a]. Isto implica que $u(t) \in D(A)$ para todo $t \in[0, a]$ e que $A u(t)=\int_{0}^{t} A R(t-s) f(s) d s$, pois $A$ é fechado. Mais ainda, da hipótese $\left(\mathbf{H}_{\alpha}\right)$ e do fato que $f \in C^{1}([0, a]:[D(A)])$ segue que

$$
u^{\prime}(t)=\int_{0}^{t} R^{\prime}(t-s) f(s) d s+f(t), \quad t \in[0, a] .
$$

Além do anterior, procedendo como na prova do Teorema 4.3.1 vemos que para $x \in D(A)$, a função $\Gamma_{2}(t)=\int_{0}^{t} N(t-s) R(s) x d s$ é diferenciável e que

$$
\Lambda_{2}^{\prime}(t)=\int_{0}^{t} N(t-s) R^{\prime}(s) x d s+N(t) x, \quad \text { q.t.p. para } t \in[0, a] .
$$

Usando as propriedades anteriores, vemos que

$$
\begin{aligned}
\frac{d}{d t}(u+N * u)(t)-A u(t)-(B * u)(t)-f(t) \\
=u^{\prime}(t)+\frac{d}{d t}(N * u)(t)-\int_{0}^{t} A R(t-s) f(s) d s-\int_{0}^{t} B(t-s) \int_{0}^{s} R(s-\xi) f(\xi) d \xi d s-f(t) \\
=\int_{0}^{t} R^{\prime}(t-s) f(s) d s+f(t)+\int_{0}^{t} N(t-s) u^{\prime}(s) d s+N(t) u(0) \\
\quad-\int_{0}^{t} A R(t-s) f(s) d s-\int_{0}^{t} B(t-s) \int_{0}^{s} R(s-\xi) f(\xi) d \xi d s-f(t) .
\end{aligned}
$$

Usando agora as equações N-resolvente, com $x=f(s)$, obtemos

$$
\begin{aligned}
\frac{d}{d t}(u+ & N * u)(t)-A u(t)-(B * u)(t)-f(t) \\
= & \int_{0}^{t}\left(A R(t-s) f(s) d s+\int_{0}^{t-s} B(t-s-\xi) R(\xi) f(s) d \xi\right) d s \\
& -\int_{0}^{t} \frac{d}{d s}\left(\int_{0}^{t-s} N(t-s-\xi) R(s-\xi)\right) f(s) d \xi d s \\
& +\int_{0}^{t} N(t-s)\left(\int_{0}^{s} R^{\prime}(s-\xi) f(\xi) d \xi+R(0) f(s)\right) d s \\
& -\int_{0}^{t} A R(t-s) f(s) d s-\int_{0}^{t} \int_{0}^{s} B(t-s) R(s-\xi) f(\xi) d \xi d s \\
= & \int_{0}^{t} \int_{0}^{t-s} B(t-s-\xi) R(\xi) f(s) d \xi d s-\int_{0}^{t} \int_{0}^{t-s} N(t-s-\xi) R^{\prime}(\xi) f(s) d \xi d s \\
& -\int_{0}^{t} N(t-s) f(s) d s+\int_{0}^{t} N(t-s) \int_{0}^{s} R^{\prime}(s-\xi) f(\xi) d \xi d s \\
& +\int_{0}^{t} N(t-s) f(s) d s-\int_{0}^{t} \int_{0}^{s} B(t-s) R(s-\xi) u(\xi) d \xi d s=0 .
\end{aligned}
$$


Portanto,

$$
\frac{d}{d t}\left(u(t)+\int_{0}^{t} N(t-s) u(s) d s\right)=A u(t)+\int_{0}^{t} B(t-s) u(s) d s+f(t), \quad t \in[0, a] .
$$

Para estudar o caso geral, precisamos de algumas estimativas sobre as soluções de 4.52. Veja que nas condições anteriores,

$$
\begin{aligned}
\| & u(t) \\
\leq & \left\|_{[D(A)]}^{t}+\right\|(u+N * u)^{\prime}(t) \| \\
& \int_{0}^{t}\|R(t-s) f(s)\| d s+2 \int_{0}^{t}\|A R(t-s) f(s)\| d s \\
& +\int_{0}^{t}\left\|B(t-s) \int_{0}^{s} R(s-\xi) f(\xi)\right\| d \xi d s+\|f(t)\| \\
\leq & \int_{0}^{t} c_{1}\|f(s)\|_{[D(A)]} d s+c_{2} \int_{0}^{t} b(t-s) \int_{0}^{s}\|f(\xi)\|_{[D(A)]} d \xi d s+\|f(t)\|,
\end{aligned}
$$

onde $c_{1}, c_{2}$ são constantes independentes de $t$ e $f$. Assim, existe $c_{3}>0$ independente de $t$ e $f \in C^{1}([0, a]:[D(A)])$ tal que

$$
\sup _{t \in[0, a]}\|u(t)\|_{[D(A)]}+\sup _{t \in[0, a]}\left\|(u+N * u)^{\prime}(t)\right\| \leq c_{3}\|f\|_{L^{1}([0, a]:[D(A)])}+\|f(\theta)\|_{a} \cdot(4.5
$$

Vejamos agora o caso geral. Seja $f \in C([0, a]: X) \cap L^{1}([0, a]:[D(A)])$ e $\left(f_{n}\right)_{n \in \mathbb{N}}$ em $C^{1}([0, a]:[D(A)])$ tal que $f_{n} \rightarrow f$ em $C([0, a]: X)$ e $f_{n} \rightarrow f$ em $L^{1}([0, a]:[D(A)])$. Como conseqüência dos passos anteriores, obtemos que $u_{n}=R * f_{n}$ é solução clássica de

$$
\begin{aligned}
\frac{d}{d t}\left(w(t)+\int_{0}^{t} N(t-s) w(s) d s\right) & =A w(t)+\int_{0}^{t} B(t-s) w(s) d s+f_{n}(t), \\
w(0) & =0 .
\end{aligned}
$$

Mais ainda, segue da desigualdade 4.53 que $\left(u_{n}\right)_{n \in \mathbb{N}}$ é convergente em $C([0, a]:[D(A)])$. Seja $u \in C([0, a]:[D(A)])$ tal que $u_{n} \rightarrow u$ em $C([0, a]:[D(A)])$. Como $N(\cdot) \in L^{1}([0, a]:$ $\mathcal{L}([D(A)]))$ e $B(\cdot) \in L^{1}([0, a]:[D(A)])$, segue que $u_{n}+N * u_{n} \rightarrow u+N * u$ e $B * u_{n} \rightarrow B * u$ convergem em $C([0, a]: X)$. Similarmente, existe $v \in C([0, a]: X)$ tal que $\frac{d}{d t}\left(u_{n}+N * u_{n}\right) \rightarrow$ $v$ em $C([0, a]: X)$. É fácil ver que nestas condições $\frac{d}{d t}(u+N * u)=v$ e portanto que $(u+N * u) \in C^{1}([0, a]: X)$. Das observações anteriores e de 4.54)-4.55 obtemos que

$$
\frac{d}{d t}\left(u(t)+\int_{0}^{t} N(t-s) u(s) d s\right)=A u(t)+\int_{0}^{t} B(t-s) u(s) d s+f(t),
$$

o que prova que $u(\cdot)$ é solução clássica.

A prova do resultado para o caso em que $f \in W^{1,1}([0, a]: X)$ é similar ao anterior, por isso, daremos uma prova resumida. 
Se $f \in W^{1,1}([0, a]: X)$ então existe $\left(f_{n}\right)_{n \in \mathbb{N}}$ em $C^{1}([0, a]:[D(A)])$ tal que $f_{n} \rightarrow f$ em $C([0, a]: X)$ e $f_{n} \rightarrow f$ em $W^{1,1}([0, a]: X)$. Da primeira parte da demonstração sabemos que para cada $n \in \mathbb{N}$, a função $u_{n}=R * f_{n}$ é solução clássica de 4.54 -4.55. Se $V(\cdot)$ é a função introduzida no Lema 4.3.1, então

$$
-V(t) f(0)=\int_{0}^{t} \frac{d}{d s}(V(t-s) f(s)) d s=-\int_{0}^{t} R(t-s) f(s) d s+\int_{0}^{t} V(t-s) f^{\prime}(s) d s,
$$

de onde segue que

$$
u(t)=V(t) f(0)+\int_{0}^{t} V(t-s) f^{\prime}(s) d s, \quad t \in[0, a] .
$$

Usando 4.56) vemos que

$$
\begin{aligned}
\|u(t)\|_{[D(A)]}+\left\|(u+N * u)^{\prime}(t)\right\|_{X} \leq & \|V(t) f(0)\|_{[D(A)]}+\int_{0}^{t}\left\|V(t-s) f^{\prime}(s)\right\|_{[D(A)]} d s \\
& \left\|A \int_{0}^{t} R(t-s) f(s) d s\right\| \\
& +\int_{0}^{t}\left\|B(t-s) \int_{0}^{s} R(s-\xi) f(\xi)\right\| d \xi d s \\
\leq & c_{4}\|f(\theta)\|_{a}+c_{5}\left\|f^{\prime}\right\|_{L^{1}([0, a]: X)},
\end{aligned}
$$

onde $c_{4}, c_{5}$ são constantes independentes de $t, f$ e $f^{\prime}$. Assim, segue que

$$
\sup _{t \in[0, a]}\|u(t)\|_{[D(A)]}+\sup _{t \in[0, a]}\left\|(u+N * u)^{\prime}(t)\right\|_{X} \leq c_{4}\|f(\theta)\|_{a}+c_{5}\left\|f^{\prime}\right\|_{L^{1}([0, a]: X)} \cdot(4.5
$$

Da desigualdade (4.57) temos que a seqüência $\left(u_{n}\right)_{n \in \mathbb{N}}$ é convergente em $C([0, a]:[D(A)])$. Seja $u \in C([0, a]:[D(A)])$ tal que $u_{n} \rightarrow u$ em $C([0, a]:[D(A)])$. Como $N(\cdot) \in L^{1}([0, a]:$ $\mathcal{L}([D(A)]))$ e $B(\cdot) \in L^{1}([0, a]:[D(A)])$ segue que $u_{n}+N * u_{n} \rightarrow u+N * u$ e $B * u_{n} \rightarrow B * u$ convergem em $C([0, a]: X)$. Por outro lado, existe $v \in C([0, a]: X)$ tal que $\frac{d}{d t}\left(u_{n}+N * u_{n}\right) \rightarrow v$ em $C([0, a]: X)$. É fácil ver que $\frac{d}{d t}(u+N * u)=v$ e portanto que $(u+N * u) \in C^{1}([0, a]: X)$. Das observações anteriores de 4.54)-4.55 obtemos

$$
\frac{d}{d t}\left(u(t)+\int_{0}^{t} N(t-s) u(s) d s\right)=A u(t)+\int_{0}^{t} B(t-s) u(s) d s+f(t)
$$

o que prova que $u(\cdot)$ é solução clássica.

Corolário 4.3.2 Sejam $f \in C([0, a]: X)$ e u(.) a solução fraca de (4.42)-(4.43). Se $u(\cdot) \in$ $C^{1}([0, a]: X)$, então u(.) é solução clássica de 44.42)-(4.43). 
Demonstração: Assuma sem perda de generalidade que $f(0)=0$. Para cada $n \in \mathbb{N}$ definimos a função $f_{n} \in C^{1}([0, a]: X)$ por

$$
f_{n}(t)=\left(\rho_{n} * f\right)(t)=\int_{0}^{t} \rho_{n}(t-s) f(s) d s, \quad t \in[0, a],
$$

onde $\left(\rho_{n}\right)_{n \in \mathbb{N}}$ é uma família de funções em $C^{\infty}(\mathbb{R})$, tais que $\rho_{n}(t) \geq 0, \rho_{n}(t)=0$ quando $t \geq \frac{a}{n}$ e $\int_{0}^{\infty} \rho_{n}(t) d t=1$

Como conseqüência do Teorema 4.3.2, sabemos que $u_{n}=R * f_{n}$ é solução clássica de

$$
\begin{aligned}
\frac{d}{d t}\left(w(t)+\int_{0}^{t} N(t-s) w(s) d s\right) & =A w(t)+\int_{0}^{t} B(t-s) w(s) d s+f_{n}(t), \\
w(0) & =0 .
\end{aligned}
$$

Mais ainda, como $u_{n}=R * f_{n}=\rho_{n} *(R * f)=\rho_{n} * u$ e $u \in C^{1}([0, a]: X)$, segue que $u_{n} \rightarrow u$ em $C^{1}([0, a]: X)$ e que $u_{n}+N * u_{n} \rightarrow u+N * u \operatorname{em~} C^{1}([0, a]: X)$.

Seja $\phi_{n, m}(t)=\left\|u_{n}(t)-u_{m}(t)\right\|_{[D(A)]}$. Usando as propriedades anteirores, vemos que para $n, m \in \mathbb{N}$ que

$$
\begin{aligned}
\phi_{n, m}(t) \leq & \left\|u_{n}(t)-u_{m}(t)\right\|+\left\|\left(u_{n}+N * u_{m}\right)^{\prime}(t)-\left(u_{n}+N * u_{m}\right)^{\prime}(t)\right\| \\
& +\int_{0}^{t} b(t-s) \phi_{n, m}(s) d s+\left\|f_{n}(t)-f_{m}(t)\right\| .
\end{aligned}
$$

Assim podemos escrever que

$$
\phi_{n, m}(t) \leq \Lambda_{n, m}(t)+\int_{0}^{t} b(t-s) \phi_{n, m}(s) d s
$$

onde $\Lambda_{n, m}(t) \rightarrow 0$ uniformemente em $[0, a]$ quando $n, m \rightarrow \infty$.

Sejam $0<t_{1}<t_{2}<\ldots<t_{n}=a$ tais que $\int_{t_{i}}^{t_{i+1}} b(s) d s \leq \frac{1}{2}$ para cada $i=1 \ldots n$.

Nestas condições, para $t \in\left[0, t_{1}\right]$, vemos que

$$
\begin{aligned}
\phi_{n, m}(t) & \leq \sup _{s \in[0, a]} \Lambda_{n, m}(s)+\int_{0}^{t} b(s) \sup _{s \in\left[0, t_{1}\right]} \phi_{n, m}(s) d s \\
& \leq \sup _{s \in[0, a]} \Lambda_{n, m}(s)+\frac{1}{2} \sup _{s \in\left[0, t_{1}\right]} \phi_{n, m}(s)
\end{aligned}
$$

e assim

$$
\sup _{s \in\left[0, t_{1}\right]} \phi_{n, m}(s) \leq 2 \sup _{s \in[0, a]} \Lambda_{n, m}(s) .
$$

Usando o anterior para $t \in\left[t_{1}, t_{2}\right]$ obtemos que

$$
\begin{aligned}
\phi_{n, m}(t) \leq & \sup _{s \in[0, a]} \Lambda_{n, m}(s)+\int_{0}^{t_{1}} b(t-s) \sup _{s \in\left[0, t_{1}\right]} \phi_{n, m}(s) d s \\
& +\int_{0}^{t-t_{1}} b(s) \sup _{s \in\left[0, t_{2}\right]} \phi_{n, m}(s) d s \\
\leq & \sup _{s \in[0, a]} \Lambda_{n, m}(s)\left(1+2\|b\|_{L^{1}}\right)+\frac{1}{2} \sup _{s \in\left[0, t_{2}\right]} \phi_{n, m}(s),
\end{aligned}
$$


e então

$$
\sup _{s \in\left[0, t_{2}\right]} \phi_{n, m}(s) \leq 2\left(1+2\|b\|_{L^{1}}\right) \sup _{s \in[0, a]} \Lambda_{n, m}(s) .
$$

É claro que após um número finito de passos obtemos a existência de $c>0$ independente de $t, n$ e $m$ tal que

$$
\sup _{s \in[0, a]} \phi_{n, m}(s) \leq c \sup _{s \in[0, a]} \Lambda_{n, m}(s)
$$

Isto mostra que $A u_{n}$ é convergente em $C([0, a]: X)$. Passando ao limite em 4.58)-4.59, obtemos que

$$
\frac{d}{d t}\left(u(t)+\int_{0}^{t} N(t-s) u(s) d s\right)=A u(t)+\int_{0}^{t} B(t-s) u(s) d s+f(t), \quad t \in[0, a],
$$

o que prova que $u(\cdot)$ é solução clássica.

\subsection{Aplicações a equações neutras com retardamento não limi- tado}

Nesta seção usaremos a teoria de operadores N-resolventes desenvolvidas na seção anterior no estudo de uma classe de equações integro-diferenciais do tipo neutro que podem ser modeladas na forma

$$
\begin{aligned}
\frac{d}{d t}\left(x(t)+\int_{-\infty}^{t} N(t-s) x(s) d s\right) & =A x(t)+\int_{0}^{t} B(t-s) x(s) d s+G\left(t, x_{t}\right), t \in(0, a) . \\
x_{0} & =\varphi
\end{aligned}
$$

onde $A,(N(t))_{t \geq 0}$ e $(B(t))_{t \geq 0}$ são operadores que verificam as condições $\left(\mathbf{H N}_{\mathbf{1}}\right)$ - $\left(\mathbf{H N}_{\mathbf{4}}\right)$; a história $x_{t}:(-\infty, 0] \rightarrow X, x_{t}(\theta)=x(t+\theta)$, pertence a um espaço de fase abstrato $\mathcal{B}$ definido axiomaticamente que verifica os axiomas (A), (A1) e (B) introduzidos no Capítulo 1 e $G:[0, a] \times \mathcal{B} \rightarrow X$ é uma função apropriada.

Para introduzir um conceito conveniente de soluções fracas para 4.60-4.61, podemos assumir que $\varphi(\cdot)$ é suficientemente regular de modo que a função $\Lambda(\cdot, \varphi)$ definida por

$$
\frac{d}{d t}\left(\int_{-\infty}^{0} N(t-s) \varphi(s) d s\right)=\Lambda(t, \varphi), \quad t \in[0, a] .
$$

exista e seja integrável sobre $[0, a]$. Neste caso, podemos dizer que uma função $u:(-\infty, a] \rightarrow$ $X$ é solução fraca de 4.60-4.61) se $u_{0}=\varphi \mathrm{e}$

$$
u(t)=R(t) \varphi(0)+\int_{0}^{t} R(t-s) G\left(s, u_{s}\right) d s+\int_{0}^{t} R(t-s) \Lambda(s, \varphi) d s, \quad t \in[0, a] .
$$


No que segue sempre assumiremos que $\varphi(\cdot)$ é tal que $\Lambda(\cdot, \varphi)$ é contínua sobre $[0, a]$.

Definição 4.4.1 Dizemos que uma função $u:(-\infty, a] \rightarrow X$ é uma solução fraca de 4.60)4.61) em $[0, b], 0<b \leq a$, se $u_{0}=\varphi ;\left.u\right|_{[0, a]} \in C([0, a]: X) e$

$$
u(t)=R(t) \varphi(0)+\int_{0}^{t} R(t-s)\left(G\left(s, u_{s}\right)+\Lambda(s, \varphi)\right) d s, \quad t \in[0, a] .
$$

As provas dos seguintes resultados de existência de soluções fracas são análogas as provas dos Teoremas 2.1.1, 2.1.3 do Capítulo 2. Por isso decidimos não incluir a demonstração.

Teorema 4.4.1 Suponha que a função $G:[0, a] \times \mathcal{B} \rightarrow X$ é contínua e que existe constante positiva $L_{G}$ tal que

$$
\left\|G\left(t, \psi_{1}\right)-G\left(t, \psi_{2}\right)\right\| \leq L_{G}\left\|\psi_{1}-\psi_{2}\right\|_{\mathcal{B}}, \quad t \in[0, a], \quad \psi_{i} \in \mathcal{B}, i=1,2 .
$$

Então existe uma única solução fraca de (4.60)-4.61) sobre $[0, b]$ para algum $0<b \leq a$.

Para o próximo resultado introduzimos a seguinte definição

Definição 4.4.2 Uma familia $N$-resolvente $(R(t))_{t \geq 0}$ é compacta, se $R(t)$ é compacto para todo $t>0$.

Teorema 4.4.2 Suponha que as seguintes condições são satisfeitas

(a) $(R(t))_{t \geq 0}$ é uma família $N$-resolvente compacta;

(b) A função $G: I \times \mathcal{B} \rightarrow X$ satisfaz as seguintes condições.

(i) A função $G(t, \cdot): \mathcal{B} \rightarrow X$ é contínua para quase todo $t \in[0, a]$ e $G(\cdot, x):[0, a] \rightarrow X$ é fortemente mensurável para todo $x \in X$;

(ii) Existe uma função contínua $m_{G}:[0, a] \rightarrow[0, \infty)$ e uma função contínua e não decrescente $\Omega_{G}:[0, \infty) \rightarrow(0, \infty)$ tal que

$$
\|G(t, \psi)\| \leq m_{G}(t) \Omega_{G}\left(\|\psi\|_{\mathcal{B}}\right), \quad(t, \psi) \in[0, a] \times \mathcal{B} .
$$

Se

$$
M K_{a} \int_{0}^{t} m_{G}(s) d s \leq \int_{C}^{\infty} \frac{d s}{\Omega_{G}(s)} ;
$$

onde $C=M_{a}\|\varphi\|+K_{a} C(a, \varphi)$, então existe uma solução fraca para o problema (4.60)(4.61) sobre $[0, a]$. 
Nos próximos resultados estudaremos a regularidade das soluções fracas, equivalentemente, assumiremos que a função $\Lambda(\cdot, \varphi) \in W^{1,1}([0, a]: X)$.

Definição 4.4.3 Uma função $u:(-\infty, a) \rightarrow X$ é uma solução clássica de (4.60)-(4.61) em $[0, b]$ se $u_{0}=\varphi$, a função $t \rightarrow u(t)+\int_{-\infty}^{t} N(t-s) u(s) d s \in C^{1}((0, b): X), u(\cdot) \in C((0, b):$ $D(A))$ e 4.60) é satisfeita.

No próximo Lema, $S(t)$ é o operador definido em 1.6$)$ e $U(t): \mathcal{B} \rightarrow \mathcal{B}$ é o operador definido por

$$
[U(t) \varphi](\theta)=\left\{\begin{aligned}
R(t+\theta) \varphi(0), & \text { se } \quad-t \leq \theta \leq 0, \\
\varphi(t+\theta), & \text { se }-\infty<\theta<-t
\end{aligned}\right.
$$

onde $(R(t))_{t \geq 0}$ é uma família N-resolvente para 4.1. 4.2 .

Lema 4.4.1 Suponha que as funções $R(\cdot) \varphi(0)$ e $S(\cdot) \varphi$ são Lipschitz em $[0, a]$. Seja y : $(-\infty, a] \rightarrow X$ é a função definida por

$$
y(t)=\left\{\begin{aligned}
R(t) \varphi(0) & \text { se } \quad 0 \leq t \leq a \\
\varphi(t) & \text { se } \quad-\infty<t<0 .
\end{aligned}\right.
$$

Então, a função $s \rightarrow y_{s}$ é Lipshitz em $[0, a]$.

Demonstração: Análoga a demonstração do Lema 2.2.1 apenas substituindo a função $W(\cdot)$ por $U(\cdot)$.

Lema 4.4.2 Assuma que as condições do Lema 4.4.1 são verificadas e suponha que existam constantes positivas $L_{G}, L_{\Lambda}>0$, tais que

$$
\begin{aligned}
\left\|G\left(t, \psi_{1}\right)-G\left(s, \psi_{2}\right)\right\| & \leq L_{F}\left(|t-s|+\left\|\psi_{1}-\psi_{2}\right\|_{\mathcal{B}}\right), \\
\|\Lambda(t, \varphi)-\Lambda(s, \varphi)\| & \leq L_{\Lambda}|t-s|
\end{aligned}
$$

para todo $t, s \in[0, b]$ e $\psi_{1}, \psi_{2} \in \mathcal{B}$. Se u(.) é uma solução fraca de 4.60)-4.61) sobre $[0, b]$, então $u(\cdot)$ é Lipschitz contínua sobre $[0, b]$.

Demonstração: Considere a decomposição $u(t)=y(t)+z(t)$, onde $y(\cdot)$ é a função introduzida em (4.64). Como $y(\cdot)$ é Lipschitz em $[0, b]$, somente mostraremos que $z(\cdot)$ é Lipschitz. 
Usando que a função $s \rightarrow y_{s}$ é Lipschitz, para $t \in[0, b)$ e $h>0$ tal que $t+h \in[0, b]$ obtemos que

$$
\begin{aligned}
\| & z(t+h)-z(t) \| \\
\leq & \int_{0}^{t}\|R(t-s)\|\|\Lambda(t+h, \varphi)-\Lambda(t, \varphi)\| d s+\int_{0}^{h}\|R(t+h-s) \Lambda(t, \varphi)\| d s \\
+ & \int_{0}^{t}\|R(t-s)\|\left\|G\left(s+h, y_{s+h}+z_{s+h}\right)-G\left(s, y_{s}+z_{s}\right)\right\| d s \\
& \quad+\int_{0}^{h}\|R(t+h-s)\|\left\|G\left(s, x_{s}\right)\right\| d s \\
\leq & C_{1} h+M \int_{0}^{t} L_{G}\left(h+\left\|y_{s+h}+z_{s+h}-y_{s}-z_{s}\right\|_{\mathcal{B}}\right) d s \\
\leq & C_{2} h+M L_{G} \int_{0}^{t}\left\|z_{s+h}-z_{s}\right\|_{\mathcal{B}} d s \\
\leq & C_{2} h+M L_{G} K_{b} \int_{0}^{t}\|z(\theta+h)-z(\theta)\|_{s} d s,
\end{aligned}
$$

onde $C_{i}, i=1,2$, são constantes independentes de $t$ e $h$. Tomando supremo em $[0, t]$ segue que

$$
\|z(\theta+h)-z(\theta)\|_{t} \leq C_{2} h+M L_{G} K_{b} \int_{0}^{t}\|z(\theta+h)-z(\theta)\|_{s} d s,
$$

O resultado é agora conseqüência da desigualdade de Gronwall-Bellman.

Teorema 4.4.3 Suponha que $X$ possui (RNP). Assuma que as hipóteses do Lema 4.4.2 são verificadas e que $\varphi(0) \in D(A)$. Se u(·) é uma solução fraca de (4.60)- 4.61) sobre [0,b], então $u(\cdot)$ é uma solução clássica de 4.60)-(4.61) sobre $[0, b]$.

Demonstração: Segue do Lema 4.4.2 que a solução fraca $u(\cdot)$ é Lipschitz sobre $[0, b]$. Como conseqüência, as funções $t \rightarrow u_{t}$ e $t \rightarrow G\left(t, u_{t}\right)$ são Lipschitz sobre $[0, b]$. Mais ainda, pelo Lema 2.2.3 obtemos que $t \rightarrow G\left(t, u_{t}\right) \in W^{1,1}([0, b]: X)$.

Como por hipótese $t \rightarrow \Lambda(t, \varphi) \in W^{1,1}([0, b]: X)$, pelo Teorema 4.3.2 podemos inferir que $u(\cdot)$ é solução clássica de

$$
\begin{aligned}
\frac{d}{d t}\left(w(t)+\int_{0}^{t} N(t-s) w(s) d s\right)= & A w(t)+\int_{0}^{t} B(t-s) w(s) d s+G\left(t, x_{t}\right) \\
& -\frac{d}{d t} \int_{-\infty}^{0} N(t-s) \varphi(s) d s, \quad t \in[0, b], \\
w(0)= & \varphi(0) .
\end{aligned}
$$

Isto nos permite afirmar que $u(\cdot)$ é solução clássica de 4.60-4.61). Agora a prova está completa. 


\section{Referências Bibliográficas}

[1] Abolinia, V. E. \& Mishkis, A. D., Mixed problems for quasilinear hyperbolic systems in the plane. Mat. Sb. 50, 423-442.

[2] Adimy, M., Bouzahir, H. \& Ezzinbi, K., Existence and stability for some partial neutral functional differential equations with infinite delay. J. Math. Anal. Appl. 294 (2004), no. 2, 438-461.

[3] Adimy, M., Ezzinbi, K. \& Laklach, M., Existence of solutions for a class of partial neutral differential equations. C. R. Acad. Sci. Paris Sér. I Math. 330 (2000), no. 11, 957-962.

[4] Adimy, M. \& Ezzinbi, K., A class of linear partial neutral functional-differential equations with nondense domain. J. Differential Equations, 147 (1998), no. 2, 285-332.

[5] Adimy, M. \& Ezzinbi, K., Strict solutions of nonlinear hyperbolic neutral differential equations. Appl. Math. Lett. 12 (1999), no. 1, 107-112.

[6] Balachandran, K. \& Dauer, J. P., Existence of solutions of nonlinear neutral integrodifferential equations in Banach spaces. J. Math. Anal. Appl. 251 (2000), no. 1, 93-105.

[7] Balachandran, K., Sakthivel, R. \& Dauer, J. P., Controllability of neutral functional integrodifferential systems in Banach spaces. Comput. Math. Appl. 39 (2000), no. $1-2$

[8] Benchohra, M.. Henderson, J. \& Ntouyas, S. K., Existence results for impulsive multivalued semilinear neutral functional differential inclusions in Banach spaces. J. Math. Anal. Appl. 263 (2001), no. 2, 763-780 
[9] Benchohra, M. \& Ntouyas, S. K., Neutral functional differential and integrodifferential inclusions in Banach spaces. Boll. Unione Mat. Ital. Sez. B Artic. Ric. Mat. (8) 4 (2001), no. 3, 767-782.

[10] Brayton, R. K., Bifurcation of periodic solutions in a nonlinear difference-differential equation of neutral type. Q. Appl. Math. 24, 215-224.

[11] Brayton, R. K. \& Moser, J. K., A theory of nonlinear networks. Q. Appl. Math. 24, 215-224.

[12] Cannarsa, P. \& Sforza, D., Global solutions of abstract semilinear parabolic equations with memory terms. NoDEA Nonlinear Differential Equations Appl. 10 (2003), no. 4, 399-430.

[13] Clément, Ph. \& Nohel, J. A., Asymptotic behavior of solutions of nonlinear Volterra equations with completely positive kernels. SIAM J. Math. Anal. 12 (1981), no. 4, $514-535$.

[14] Clément, Ph. \& Da Prato G., Some results on nonlinear heat equations for materials of fading memory type. Journal of Integral Equations and Appl. v. 2 (1990) no. 3, $375-391$

[15] Cooke, K. L. \& Krumme, D. W., Differential-difference equations and nonlinear initial-boundary value problems for linear hyperbolic partial differential equations. J. Math. Anal. Appl. 24, 372-387.

[16] Corduneanu, C. \& Lakshmikantham, V., Equations with unbounded delay: a survey. Nonlinear Anal 4 (1980), no. 5, 831-877.

[17] Cruz, M. A. \& Hale, J. K., Stability of functional differential equations of neutral type. J. Diff. Eqs. 7, 334-355.

[18] Da Prato, G. \& Iannelli, M., Existence and regularity for a class of integrodifferential equations of parabolic type. J. Math. Anal. Appl. 112 (1985), no. 1, 36-55.

[19] Datko, R., Linear autonomous neutral differential equations in a Banach space. $J$. Diff. Equations, 25 (1977), no. 2, 258-274. 
[20] Diestel, J. \& Uhl Jr, J.J., Vector Measure. Mathematical Surveys and Monographs, American Mathematic Society, Volume 14.

[21] Engel, K. J. \& Nagel, R., One-Parameter Semigroups for Linear Evolution Equations. Springer, New York, 1999.

[22] Fu, X. \& Ezzinbi, K., Existence of solutions for neutral functional differential evolution equations with nonlocal conditions. Nonlinear Anal. 54 (2003), no. 2, $215-227$.

[23] Granas, A. \& Dugundji, J., Fixed Point Theory. Springer-Verlag, New York, 2003.

[24] Grimmer, R. C. \& Kappel, F., Series expasions for resolvents of volterra integrodifferential equations in Banch space. Siam Journal of Math. Anal. 15 (1984), no. $3,595-604$.

[25] Grimmer, R. C. \& Pritchard, A. J., Analytic resolvent operators for integral equations in Banach space. J. Differential Equations 50 (1983), no. 2, 234-259.

[26] Grimmer, R. \& Prüss, J., On linear Volterra equations in Banach spaces. Hyperbolic partial differential equations, II. Comput. Math. Appl. 11 (1985), no. 1-3, 189-205.

[27] Gurtin, M.E. \& Pipkin, A. C., A general theory of heat conduction with finite wave speed, Arch. Rat. Mech. Anal. 31 (1968), 113-126.

[28] Hale, J. K., Partial neutral functional-differential equations. Rev. Roumaine Math. Pures Appl. 39 (1994), no. 4, 339-344.

[29] Hale, J. K., Verduyn, L. \& Sjoerd M., Introduction to functional-differential equations. Applied Mathematical Sciences, 99. Springer-Verlag, New York, 1993.

[30] Hale, J. K. \& Kato, J., Phase space for retarded equations with infinite delay. Funkcial. Ekvac. 21 (1978), no. 1, 11-41.

[31] Henríquez, H. R. \& Hernández, E., On the abstract Cauchy problem in Fréchet spaces. Proc. Amer. Math. Soc. 115 (1992), no. 2, 353-360.

[32] Henríquez, H. R., Existence of periodic solutions of neutral functional differential equations with unbounded delay. Proyecciones. 19 (2000), no. 3, 305-329. 
[33] Henríquez H. R., Regularity of solutions of abstract retarded functional-differential equations with unbounded delay. Nonlinear Anal. 28 (1997), no. 3, 513-531.

[34] Hernández E., Massera type criterion for a partial neutral functional differential equation. Electron. J. Differential Equations. 2002, no. 40, 17 pp. (electronic).

[35] Hernández, E. \& Henríquez, H. R., Existence of periodic solutions of partial neutral functional-differential equations with unbounded delay. J. Math. Anal. Appl. 221 (1998), no. 2, 499-522.

[36] Hernández, E. \& Pelicer, M., Asymptotically almost periodic and almost periodic solutions for partial neutral differential equations. Appl. Math. Lett. 18 (2005), no. $11,1265-1272$.

[37] Hernández, E. \& Diagana, T., Existence and Uniqueness of Pseudo Almost Periodic Solutions to Some Abstract Partial Neutral Functional-Differential Equations. Submetido para publicação.

[38] Hernández, E. \& Diagana, T., On Pseudo Almost Periodic Solutions for Neutral Functional-Differential Equations. Submetido para publicação.

[39] Hernández, E. \& Diagana, T., Pseudo Almost Periodic Solutions for Abstract Partial Neutral Integrodifferential Equations. Submetido para publicação.

[40] Hernández, E., Henríquez H. R. \& Rabello, M., Existence of Solutions for Impulsive Partial Neutral Functional Differential Equations. Submetido para publicação.

[41] Hernández, E. \& Henríquez, Hernán., Impulsive partial neutral differential equations. Appl. Math. Lett. 19 (2006), no. 3, 215-222.

[42] Hernández, E., Pierri M. \& Táboas, P., A comment on the papers: " A study on controllability of semilinear integrodifferential systems in Banach spaces " [ Comput. Math. Appl. 47 (2004), no. 4-5] and "Controllability of neutral functional integrodifferential systems in Banach spaces " [ Comput. Math. Appl. 39 (2000), no. 1-2]". Comput. Math. Appl. 50 (2005), no. 8-9, 1291-1292.

[43] Hernández, E., Existence results for partial neutral functional differential equations with nonlocal conditions. Dynam. Systems Appl. 11 (2002), no. 2, 241-252. 
[44] Hernández, E. \& Henríquez, H. R., Existence results for partial neutral functional differential equations with unbounded delay. J. Math. Anal. Appl. 221 (1998), no. $2,452-475$.

[45] Hernández, E., Regularity of solutions of partial neutral functional differential equations with unbounded delay. Proyecciones 21 (2002), no. 1, 65-95.

[46] Hernández, E., Existence results for partial neutral functional integrodifferential equations with unbounded delay. J. Math. Anal. Appl. 292 (2004), no. 1, 194-210.

[47] Hernández, E., dos Santos, J. P. C. \& Pelicer, M. L., "Asymptotically almost periodic and Almost Periodic Solutions for a class of Evolution Equation "Electron. J. Diff. Eqns. Vol. 2004(2004), no. 61, pp. 1-15.

[48] Hille, E. e Phillips, R. S., Functional Analysis and Semigroups. Third printing of the revised edition of 1957. American Mathematical Society Colloquium Publications, Vol. XXXI.

[49] Hino, Y., Murakami, S. e Naito, T., Functional Differential Equations With Infinite Delay. Lecture Notes in Mathematics, 1473. Springer-Verlag, Berlin, 1991.

[50] Komura, Y., Differentiability of nonlinear semigroups. J. Math. Soc. Japan 211969 $375-402$.

[51] Lizama, C., On Volterra equations associated with a linear operator. Proc. Amer. Math. Soc. 118 (1993), no. 4, 1159-1166.

[52] Lopes, O., Forced oscillations in nonlinear neutral differential equations. SIAM J. Appl. Math. 29, 196-201.

[53] Lopes, O., Stability and forced oscillations. J. Math. Anal. Appl. 55, 686-698.

[54] Hale J. K., Partial neutral functional-differential equations. Rev. Roumaine Math Pures Appl. 39 (1994), no. 4, 339-344

[55] Lunardi, A., Analytic Semigroups and Optimal Regularity in Parabolic Problems, PNLDE Vol. 16 , Birkhäauser Verlag, Basel, 1995.

[56] Lunardi, A., On the linear heat equation with fading memory. SIAM J. Math. Anal. 21 (1990), no. 5, 1213-1224. 
[57] Lunardi, A., Laplace transform methods in integrodifferential equations, J. Integral Equations Applications. 10 (1985), 185-211.

[58] Marle, C., M. Mesures et Probabilités. Hermann, Paris, 1974.

[59] Martin, R. H., Nonlinear Operators and Differential Equations in Banach Spaces, Robert E. Krieger Publ. Co., Florida, 1987.

[60] Miller, R. K., An integro-differential equation for rigid heat conductors with memory. J. Math. Anal. Appl. 66 (1978), no. 2, 313-332.

[61] Nachbin, L., Introduction to Functional Analysis: Banach Spaces and Differential Calculus. Translated from the Portuguese by Richard M. Aron. Monographs and Textbooks in Pure and Applied Math., 60. Marcel Dekker, Inc., New York, 1981.

[62] Nunziato, J. W., On heat conduction in materials with memory. Quart. Appl. Math. 29 (1971), 187-204.

[63] Pazy A., Semigroups of Linear Operators and Applications to Partial Differential Equations. Applied mathematical Sciences, 44. Springer-Verlag, New York-Berlin, 1983.

[64] Rankin III, S. M., Semilinear evolution equations in Banach spaces with application to parabolic partial differential equations. Trans. Amer. Math. Soc. 336 (1993), no. $2,523-535$.

[65] Sforza, D., Existence in the large for a semilinear integrodifferential equation with infinite delay. J. Differential Equations. 120 (1995), no. 2, 289-303

[66] Sforza, D., On the Hille-Yosida theorem for integro-differential equations in Banach spaces. Boll. Un. Mat. Ital. C (6) 5 (1986), no. 1, 169-173 (1987).

[67] Travis, C. C. \& Webb, G. F., Existence and stability for partial functional differential equations. Trans. Amer. Soc. 200 (1974), 395-418.

[68] Travis, C. C. \& Webb, G. F., Partial differential equations with deviating arguments in the time variable. J. Math. Anal. Appl, 56 (1976), no. 2, 397-409. 
[69] Travis, C. C. \& Webb, G. F., Existence, stability, and compactness in the $\alpha$-norm for partial functional differential equations. Trans. Amer. Math. Soc. 240 (1978), $129-143$.

[70] Travis,C. C. \& Webb G. F., Cosine families and abstract nonlinear second order differential equations. Acta Math. Acad. Sci. Hungaricae, 32 (1978) 76-96.

[71] Travis, C. C. \& Webb, G. F., Compactness, regularity, and uniform continuity properties of strongly continuous cosine families. Houston J. Math. 3 (4) (1977) $555-567$.

[72] Wu, J., Theory and Applications of Partial Functional-Differential Equations. Applied Mathematical Sciences, 119. Springer-Verlag, New York, 1996.

[73] Wu, J. \& Xia, H., Rotating waves in neutral partial functional-differential equations. J. Dynam. Differential Equations. 11 (1999), no. 2, 209-238.

[74] Wu, J. \& Xia H., Self-sustained oscillations in a ring array of coupled lossless transmission lines. J. Differential Equations, 124 (1996), no. 1, 247-278. 